Supporting Information

\title{
Synthesis and Structural Features of Quadruple Helicenes: Highly Distorted $\pi$-Systems Enabled by Accumulation of Helical Repulsions
}

\author{
Takao Fujikawa, ${ }^{a}$ Yasutomo Segawa, ${ }^{\star a, b}$ and Kenichiro Itami ${ }^{\star a, b, c}$ \\ ${ }^{a}$ Graduate School of Science, Nagoya University, Japan \\ ${ }^{b}$ JST, ERATO, Itami Molecular Nanocarbon Project, Nagoya University, Japan. \\ ${ }^{c}$ Institute of Transformative Bio-Molecules (WPI-ITbM), Nagoya University, Japan \\ E-mail: ysegawa@nagoya-u.jp (Y.S.), itami@chem.nagoya-u.ac.jp (K.I.)
}

\section{Table of Contents}

1. General

2. Synthesis of 2, 5, QH-A, and QH-B

3. Enantiomer Separation by HPLC 


\section{General}

Unless otherwise noted, all materials including dry solvents were obtained from commercial suppliers and used without further purification. 3,6-Dibromonaphthalene-2,7-diyl bis(trifluoromethanesulfonate) ${ }^{\mathrm{S} 1}$ was prepared according to the procedures reported in the literature. Unless otherwise noted, all reactions were performed with dry solvents under an atmosphere of nitrogen in dried glassware with standard vacuum-line techniques. All work-up and purification procedures were carried out with reagent-grade solvents in air.

Analytical thin-layer chromatography (TLC) was performed using E. Merck silica gel $60 \mathrm{~F}_{254}$ precoated plates $(0.25 \mathrm{~mm})$. The developed chromatogram was analyzed by UV lamp $(254 \mathrm{~nm}$ and $365 \mathrm{~nm}$ ). High-resolution mass spectra (HRMS) were obtained from a MSI.TOKYO Inc. MULTUM-FAB (FAB) and a JEOL JMS-S3000 SpiralTOF (MALDI-TOF MS). Melting points were measured on a MPA100 Optimelt automated melting point system. Chiral HPLC analysis was conducted on a Shimadzu Prominence 2000 instrument equipped with COSMOSIL Cholester column $(10 \mathrm{~mm} \times 250 \mathrm{~mm})$. Cyclic voltammetry (CV) measurements were performed by BAS ALS-600D Electrochemical Analyzer. Nuclear magnetic resonance (NMR) spectra were recorded on a JEOL JNM-ECA-600 ( $\left.{ }^{1} \mathrm{H} 600 \mathrm{MHz},{ }^{13} \mathrm{C} 150 \mathrm{MHz}\right)$ spectrometer or a JEOL ECA 600II spectrometer with Ultra $\mathrm{COOL}^{\mathrm{TM}}$ probe $\left({ }^{1} \mathrm{H} 600 \mathrm{MHz},{ }^{13} \mathrm{C} 150 \mathrm{MHz}\right)$. Chemical shifts for ${ }^{1} \mathrm{H}$ NMR are expressed in parts per million (ppm) relative to $\mathrm{CHCl}_{3}(\delta 7.26 \mathrm{ppm})$ or $\mathrm{C}_{2} \mathrm{DHCl}_{4}(\delta 6.00 \mathrm{ppm})$. Chemical shifts for

${ }^{13} \mathrm{C}$ NMR are expressed in ppm relative to $\mathrm{CDCl}_{3}(\delta 77.16 \mathrm{ppm})$ or $\mathrm{C}_{2} \mathrm{D}_{2} \mathrm{Cl}_{4}(\delta 73.78 \mathrm{ppm})$. Data are reported as follows: chemical shift, multiplicity $(\mathrm{s}=$ singlet, $\mathrm{d}=$ doublet, $\mathrm{t}=$ triplet, $\mathrm{m}=$ multiplet, $\mathrm{br}$ = broad signal), coupling constant $(\mathrm{Hz})$, and integration. 


\section{Synthesis of 2, 5, QH-A, and QH-B}

\section{Synthesis of 2-(2-bromophenyl)-5-butylthiophene (2)}

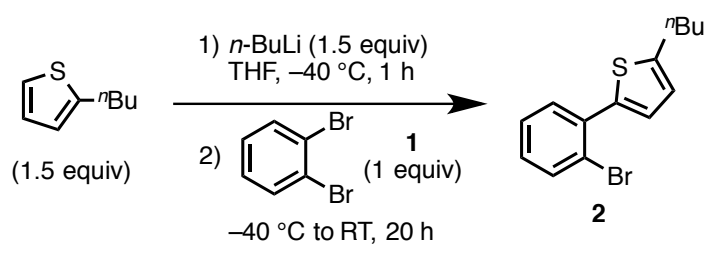

To a solution of 2-butylthiophene ( $8.42 \mathrm{~g}, 60.0 \mathrm{mmol}, 1.5$ equiv) in THF $(100 \mathrm{~mL})$ was added a pentane solution of $n$-butyllithium (1.6 M, $37.5 \mathrm{~mL}, 60.0 \mathrm{mmol}, 1.5$ equiv) at $-40{ }^{\circ} \mathrm{C}$ dropwise over $20 \mathrm{~min}$, and the mixture was stirred for $1 \mathrm{~h}$ under nitrogen. 1,2-Dibromobenzene (1) $(9.44 \mathrm{~g}, 40.0$ mmol, 1 equiv) was added dropwise over $10 \mathrm{~min}$, and then the reaction mixture was allowed to warm to room temperature. After stirring the mixture for $20 \mathrm{~h}$, the reaction was quenched by the addition of saturated $\mathrm{NH}_{4} \mathrm{Cl}$ aqueous solution. The organic layer was extracted with $\mathrm{Et}_{2} \mathrm{O}$, washed with brine, dried over $\mathrm{Na}_{2} \mathrm{SO}_{4}$, and then evaporated in vacuo. The crude material was purified by silica gel column chromatography (eluent: hexane) to afford $2(11.5 \mathrm{~g}, 97 \%)$ as a colorless oil.

${ }^{1} \mathrm{H}$ NMR (600 MHz, $\left.\mathrm{CDCl}_{3}\right) \delta 7.65(\mathrm{dd}, J=8.0,1.2 \mathrm{~Hz}, 1 \mathrm{H}), 7.46(\mathrm{dd}, J=7.7,1.6 \mathrm{~Hz}, 1 \mathrm{H}), 7.30$ (ddd, $J=7.5,7.5,1.2 \mathrm{~Hz}, 1 \mathrm{H}), 7.14(\mathrm{ddd}, J=7.9,7.4,1.7 \mathrm{~Hz}, 1 \mathrm{H}), 7.12(\mathrm{~d}, J=3.5 \mathrm{~Hz}, 1 \mathrm{H}), 6.77(\mathrm{dt}, J=$ 3.5, $0.9 \mathrm{~Hz}, 1 \mathrm{H}$ ), 2.84 (t, $J=7.7 \mathrm{~Hz}, 2 \mathrm{H}$ ), 1.71 (quintet, $J=7.6 \mathrm{~Hz}, 2 \mathrm{H}$ ), 1.44 (sextet, $J=7.4 \mathrm{~Hz}, 2 \mathrm{H}$ ), $0.96(\mathrm{t}, J=7.4 \mathrm{~Hz}, 3 \mathrm{H}) ;{ }^{13} \mathrm{C} \mathrm{NMR}\left(150 \mathrm{MHz}, \mathrm{CDCl}_{3}\right) \delta 147.1\left(4^{\circ}\right), 139.1\left(4^{\circ}\right), 135.8\left(4^{\circ}\right), 133.8(\mathrm{CH})$, $131.9(\mathrm{CH}), 128.7(\mathrm{CH}), 127.6(\mathrm{CH}), 127.5(\mathrm{CH}), 124.1(\mathrm{CH}), 122.7(\mathrm{CH}), 33.9\left(\mathrm{CH}_{2}\right), 30.0\left(\mathrm{CH}_{2}\right)$, $22.4\left(\mathrm{CH}_{2}\right), 14.0\left(\mathrm{CH}_{3}\right)$; HRMS (FAB) $m / z$ calcd for $\mathrm{C}_{14} \mathrm{H}_{15} \mathrm{BrS}[\mathrm{M}+1]^{+}: 294.0078$, found: 294.0087 .

\section{Synthesis of 2,3,6,7-tetrakis(2-(5-butylthiophen-2-yl)phenyl)naphthalene (5)}
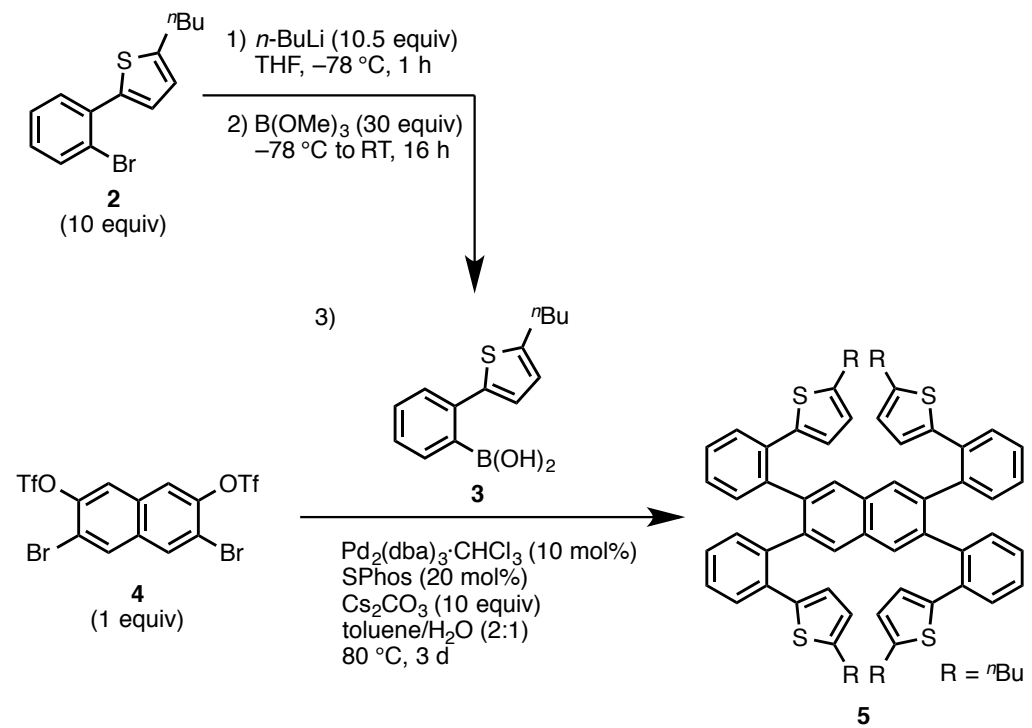

To a solution of $2(5.90 \mathrm{~g}, 20.0 \mathrm{mmol}, 10$ equiv) in THF $(100 \mathrm{~mL})$ was added a pentane solution of $n$-butyllithium (1.6 M, $13.1 \mathrm{~mL}, 21.0 \mathrm{mmol}, 10.5$ equiv) at $-78{ }^{\circ} \mathrm{C}$ dropwise over $10 \mathrm{~min}$, and the mixture was stirred for $1 \mathrm{~h}$ under nitrogen. Trimethoxyborane $(6.69 \mathrm{~mL}, 60.0 \mathrm{mmol}, 30$ equiv) was 
added, and then the reaction mixture was allowed to warm to room temperature. After stirring the mixture for $16 \mathrm{~h}$, the reaction was quenched by the addition of saturated $\mathrm{NH}_{4} \mathrm{Cl}$ aqueous solution. The organic layer was extracted with $\mathrm{Et}_{2} \mathrm{O}$, washed with brine, dried over $\mathrm{Na}_{2} \mathrm{SO}_{4}$, and then evaporated in vacuo to afford a crude mixture of (2-(5-butylthiophen-2-yl)phenyl)boronic acid (3) (5.23 g, quant) as a white slurry. Compound $\mathbf{3}$ was used in next step without further purification.

To a solution of 3,6-dibromonaphthalene-2,7-diyl bis(trifluoromethanesulfonate) (4) (1.16 g, 2.00 mmol, 1 equiv), 3, $\mathrm{Pd}_{2}(\mathrm{dba})_{3} \cdot \mathrm{CHCl}_{3}(207 \mathrm{mg}, 0.20 \mathrm{mmol}, 10 \mathrm{~mol} \%$ ), and SPhos (164 mg, $0.40 \mathrm{mmol}$, $20 \mathrm{~mol} \%)$ in toluene $(10 \mathrm{~mL})$ was added a solution of $\mathrm{Cs}_{2} \mathrm{CO}_{3}\left(6.52 \mathrm{~g}, 20.0 \mathrm{mmol}, 10\right.$ equiv) in $\mathrm{H}_{2} \mathrm{O}$ $\left(5 \mathrm{~mL}\right.$ ), and the mixture was stirred at $80{ }^{\circ} \mathrm{C}$ for 3 days under nitrogen. After cooling the mixture to room temperature, the reaction mixture was quenched by the addition of $2 \mathrm{~N} \mathrm{HCl}$ aqueous solution. The organic layer was extracted with ethyl acetate, washed with brine, dried over $\mathrm{Na}_{2} \mathrm{SO}_{4}$, and then evaporated in vacuo. $\mathrm{CHCl}_{3}$ solution of the crude material was passed through a pad of silica gel and dried again. Recrystallization from $\mathrm{CHCl}_{3} / n$-hexane bilayer system afforded $\mathbf{5}(1.42 \mathrm{~g}, 72 \%)$ as a pale yellow crystal.

${ }^{1} \mathrm{H}$ NMR $\left(600 \mathrm{MHz}, \mathrm{C}_{2} \mathrm{D}_{2} \mathrm{Cl}_{4}, 130{ }^{\circ} \mathrm{C}\right) \delta 7.81(\mathrm{~s}, 4 \mathrm{H}), 7.34(\mathrm{dd}, J=7.7,0.8 \mathrm{~Hz}, 4 \mathrm{H}), 7.19(\mathrm{td}, J=7.6$, $1.3 \mathrm{~Hz}, 4 \mathrm{H}), 7.02(\mathrm{td}, J=7.5,1.1 \mathrm{~Hz}, 4 \mathrm{H}), 6.75(\mathrm{~d}, J=7.6 \mathrm{~Hz}, 4 \mathrm{H}), 6.53(\mathrm{~d}, J=3.8 \mathrm{~Hz}, 4 \mathrm{H}), 6.31$ (d, $J=3.8 \mathrm{~Hz}, 4 \mathrm{H}$ ), 2.80 (t, $J=7.6 \mathrm{~Hz}, 8 \mathrm{H}$ ), 1.71 (quintet, $J=7.4 \mathrm{~Hz}, 8 \mathrm{H}$ ), 1.49 (sextet, $J=7.4 \mathrm{~Hz}, 8 \mathrm{H}$ ), $1.01(\mathrm{t}, J=7.4 \mathrm{H}, 12 \mathrm{H}) ;{ }^{13} \mathrm{C}$ NMR $\left(150 \mathrm{MHz}, \mathrm{C}_{2} \mathrm{D}_{2} \mathrm{Cl}_{4}, 130{ }^{\circ} \mathrm{C}\right) \delta 145.5\left(4^{\circ}\right), 141.0\left(4^{\circ}\right), 139.7\left(4^{\circ}\right)$, $138.8\left(4^{\circ}\right), 133.7\left(4^{\circ}\right), 132.2\left(4^{\circ}\right), 131.2(\mathrm{CH}), 129.9(\mathrm{CH}), 128.3(\mathrm{CH}), 126.7(\mathrm{CH}), 126.0(\mathrm{CH}), 125.9$ $(\mathrm{CH}), 123.5(\mathrm{CH}), 33.4\left(\mathrm{CH}_{2}\right), 29.4\left(\mathrm{CH}_{2}\right), 21.8\left(\mathrm{CH}_{2}\right), 13.3\left(\mathrm{CH}_{3}\right)$; HRMS (MALDI-TOF MS) $\mathrm{m} / z$ calcd for $\mathrm{C}_{66} \mathrm{H}_{64} \mathrm{~S}_{4} \mathrm{Na}[\mathrm{M}+\mathrm{Na}]^{+}$: 1007.3789 , found: 1007.3797 ; mp: $186-187{ }^{\circ} \mathrm{C}$ (recrystallized from dichloromethane/ $n$-hexane).

\section{Synthesis of QH-A}

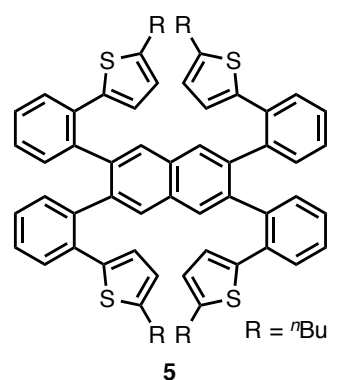

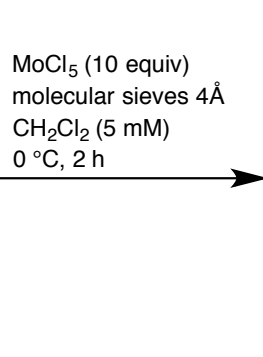

alar sieves $\mathrm{H}_{2} \mathrm{Cl}_{2}(5 \mathrm{mM})$

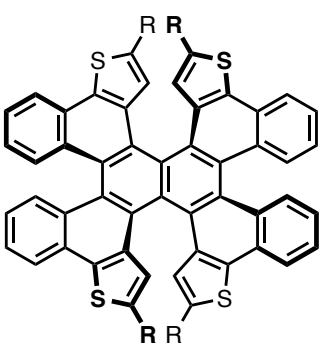

$(P, P)-(P, P)-\mathbf{Q H}$

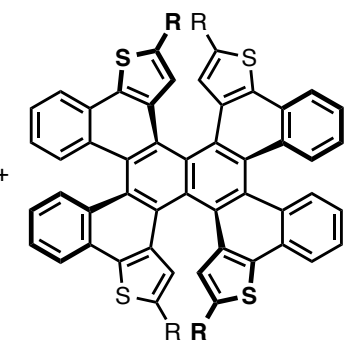

$(M, M)-(M, M)-\mathbf{Q H}$

Molecular sieves $4 \AA(1.00 \mathrm{~g})$ placed in the $100 \mathrm{~mL}$ Schlenk flask was dried in vacuo with heating by heat gun. After cooling, nitrogen was backfilled to the flask, and 5 ( $246 \mathrm{mg}, 0.250 \mathrm{mmol}, 1$ equiv) and dry $\mathrm{CH}_{2} \mathrm{Cl}_{2}(50 \mathrm{~mL})$ was added. Fresh $\mathrm{MoCl}_{5}(683 \mathrm{mg}, 2.50 \mathrm{mmol}, 10$ equiv) was added to the flask under gentle nitrogen flow at $0{ }^{\circ} \mathrm{C}$ and the reaction mixture was stirred for $2 \mathrm{~h}$. The reaction was quenched by the addition of $\mathrm{MeOH} / \mathrm{CHCl}_{3}(1: 1,40 \mathrm{~mL})$ solution and the resultant solution was directly passed through a pad of silica-gel with $\mathrm{CHCl}_{3}$ eluent. The filtrate was washed with $\mathrm{NaHCO}_{3}$ 
aqueous solution, dried over $\mathrm{Na}_{2} \mathrm{SO}_{4}$, and then evaporated in vacuo. The crude material was purified by silica-gel column chromatography (eluent: hexane/ $\mathrm{CH}_{2} \mathrm{Cl}_{2}=100: 0$ to $90: 10$ ) to afford QH-A (140 $\mathrm{mg}, 57 \%)$ as a orange solid.

${ }^{1} \mathrm{H}$ NMR $\left(600 \mathrm{MHz}, \mathrm{CDCl}_{3}\right) \delta 8.32(\mathrm{~d}, J=8.3 \mathrm{~Hz}, 4 \mathrm{H}), 8.10(\mathrm{dd}, J=8.0,0.9 \mathrm{~Hz}, 4 \mathrm{H}), 7.57(\mathrm{ddd}, J=$ 8.0, 6.9, $0.9 \mathrm{~Hz}, 4 \mathrm{H}), 7.21$ (ddd, $J=8.3,6.9,1.3 \mathrm{~Hz}, 4 \mathrm{H}), 6.13(\mathrm{~s}, 4 \mathrm{H}), 2.51\left(\mathrm{dt},{ }^{2} J=15.1,{ }^{3} J=7.4 \mathrm{~Hz}\right.$, $4 \mathrm{H}), 2.44\left(\mathrm{dt},{ }^{3} J=15.2,{ }^{3} J=7.4 \mathrm{~Hz}, 4 \mathrm{H}\right), 1.38-1.27$ (m, 8H), 1.14 (sextet, $\left.J=7.4 \mathrm{~Hz}, 8 \mathrm{H}\right), 0.83$ (t, $J=$ $7.4 \mathrm{~Hz}, 12 \mathrm{H}) ;{ }^{13} \mathrm{C}$ NMR $\left(150 \mathrm{MHz}, \mathrm{CDCl}_{3}\right) \delta 143.5\left(4^{\circ}\right), 137.3\left(4^{\circ}\right), 133.5\left(4^{\circ}\right), 131.7(\mathrm{CH}), 129.2\left(4^{\circ}\right)$, $128.1\left(4^{\circ}\right), 126.9(\mathrm{CH}), 126.6\left(4^{\circ}\right), 125.9\left(4^{\circ}\right), 125.7\left(4^{\circ}\right), 124.6(\mathrm{CH}), 123.5(\mathrm{CH}), 123.4(\mathrm{CH}), 33.6$ $\left(\mathrm{CH}_{2}\right), 29.9\left(\mathrm{CH}_{2}\right), 22.0\left(\mathrm{CH}_{2}\right), 14.1\left(\mathrm{CH}_{3}\right)$; HRMS (MALDI-TOF MS) $m / z$ calcd for $\mathrm{C}_{66} \mathrm{H}_{56} \mathrm{~S}_{4}[\mathrm{M}]^{+}$: 976.3265, found: 976.3264 ; $\mathrm{mp}:>300^{\circ} \mathrm{C}$.

\section{Synthesis of QH-B}

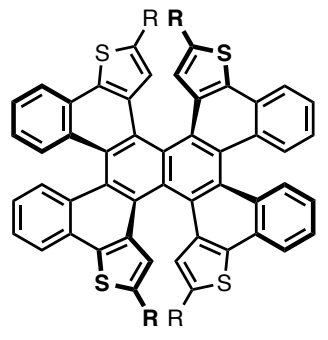

$(P, P)-(P, P)-\mathbf{Q H}$

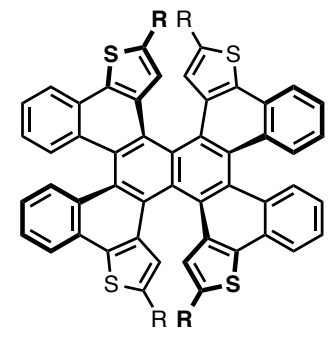

$(M, M)-(M, M)-\mathbf{Q H}$

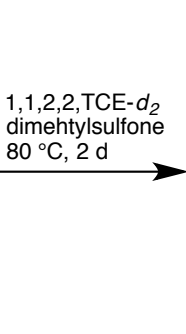
dimehtylsulfone

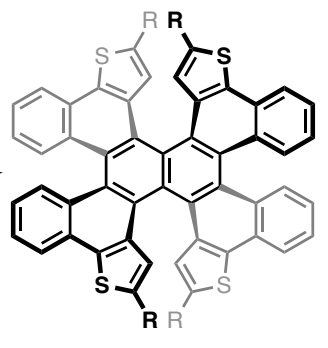

$(P, P)-(M, M)-\mathbf{Q H}$

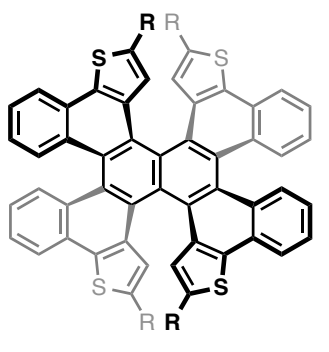

$(M, M)-(P, P)-\mathbf{Q H}$

A solution of QH-A (ca. $3.2 \mathrm{mg}, 0.0033 \mathrm{mmol})$ in 1,1,2,2-tetrachloroehtane- $d_{2}(0.4 \mathrm{~mL})$ and dimethylsulfone ( $c a .0 .28 \mathrm{mg}$ ) in NMR tube was warmed to $80{ }^{\circ} \mathrm{C}$ for 2 days and then analyzed by ${ }^{1} \mathrm{H}$ NMR at room temperature. Using dimethylsulfone as an internal standard, NMR yield of QH-B was determined to $100 \%$.

${ }^{1} \mathrm{H}$ NMR $\left(600 \mathrm{MHz}, \mathrm{CDCl}_{3}\right) \delta 8.55(\mathrm{~d}, J=8.6 \mathrm{~Hz}, 4 \mathrm{H}), 8.13(\mathrm{dd}, J=8.0,0.8 \mathrm{~Hz}, 4 \mathrm{H}), 7.52(\mathrm{ddd}, J=$ 8.1, 6.9, $1.1 \mathrm{~Hz}, 4 \mathrm{H}), 7.29$ (ddd, $J=8.6,6.8,1.3 \mathrm{~Hz}, 4 \mathrm{H}), 7.28$ (s, 4H), 2.67 (t, $J=7.4 \mathrm{~Hz}, 8 \mathrm{H}), 1.52-$ $1.47(\mathrm{~m}, 8 \mathrm{H}), 1.29-1.18(\mathrm{~m}, 8 \mathrm{H}), 0.84(\mathrm{t}, J=7.3 \mathrm{~Hz}, 12 \mathrm{H}) ;{ }^{13} \mathrm{C} \mathrm{NMR}\left(150 \mathrm{MHz}, \mathrm{CDCl}_{3}\right) \delta 144.8\left(4^{\circ}\right)$, $137.5\left(4^{\circ}\right), 133.7\left(4^{\circ}\right), 132.7\left(4^{\circ}\right), 131.5(\mathrm{CH}), 129.1\left(4^{\circ}\right), 128.6\left(4^{\circ}\right), 126.7(\mathrm{CH}), 125.7\left(4^{\circ}\right), 124.9$ $(\mathrm{CH}), 124.8\left(4^{\circ}\right), 124.2(\mathrm{CH}), 124.1(\mathrm{CH}), 33.7\left(\mathrm{CH}_{2}\right), 30.4\left(\mathrm{CH}_{2}\right), 22.0\left(\mathrm{CH}_{2}\right), 14.0\left(\mathrm{CH}_{3}\right)$; HRMS (MALDI-TOF MS) $\mathrm{m} / \mathrm{z}$ calcd for $\mathrm{C}_{66} \mathrm{H}_{56} \mathrm{~S}_{4}[\mathrm{M}]^{+}:$976.3265, found: 976.3266 ; mp: $>300{ }^{\circ} \mathrm{C}$. 


\section{Enantiomer Separation by HPLC}

HPLC analysis was conducted on a Shimadzu Prominence 2000 instrument equipped with COSMOSIL Cholester column $(10 \mathrm{~mm}$ x $250 \mathrm{~mm})$. Eluted products were analyzed at $400 \mathrm{~nm}$. Flow rate was $3.0 \mathrm{~mL} / \mathrm{min}$ and temperature was $30{ }^{\circ} \mathrm{C}$. Eluted by chloroform/2-propanol (3:7)

(a)

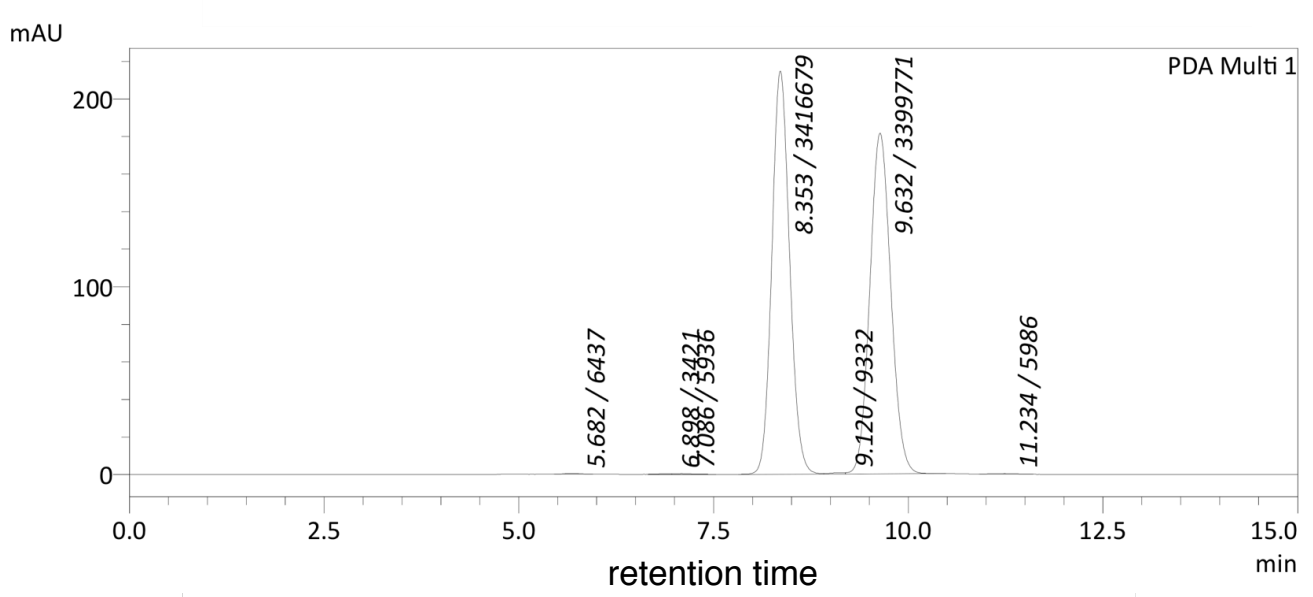

(b) mAU

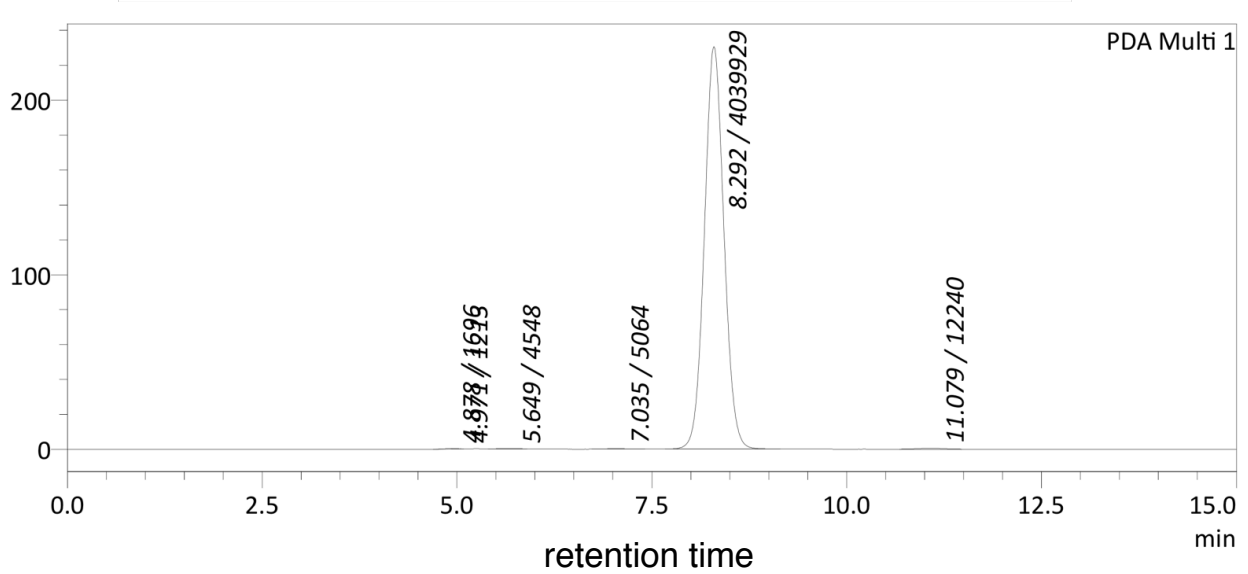

Figure S1. HPLC analysis of (a) the racemic mixture of QH-A and (b) the separated (+)-QH-A. 
(a) $\mathrm{mAU}$

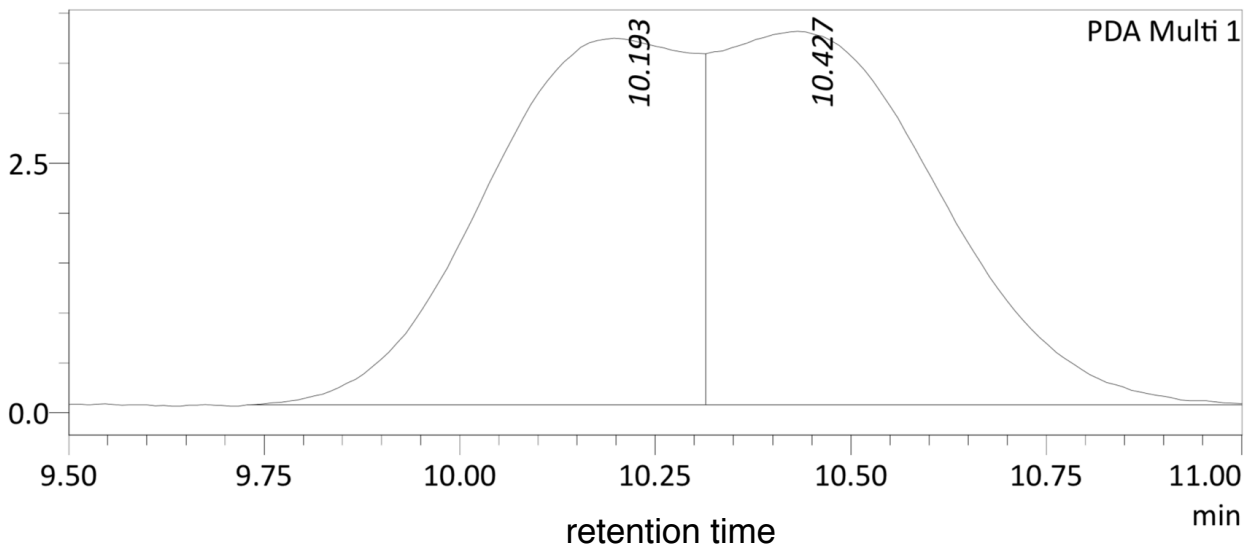

(b) $\mathrm{mAU}$

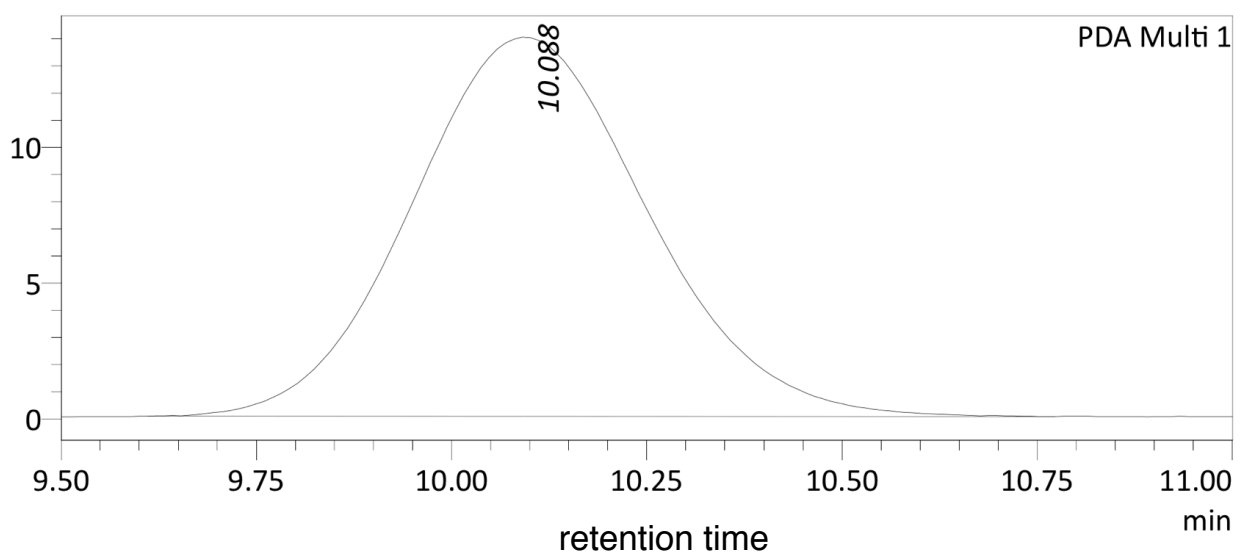

(c) $\mathrm{mAU}$

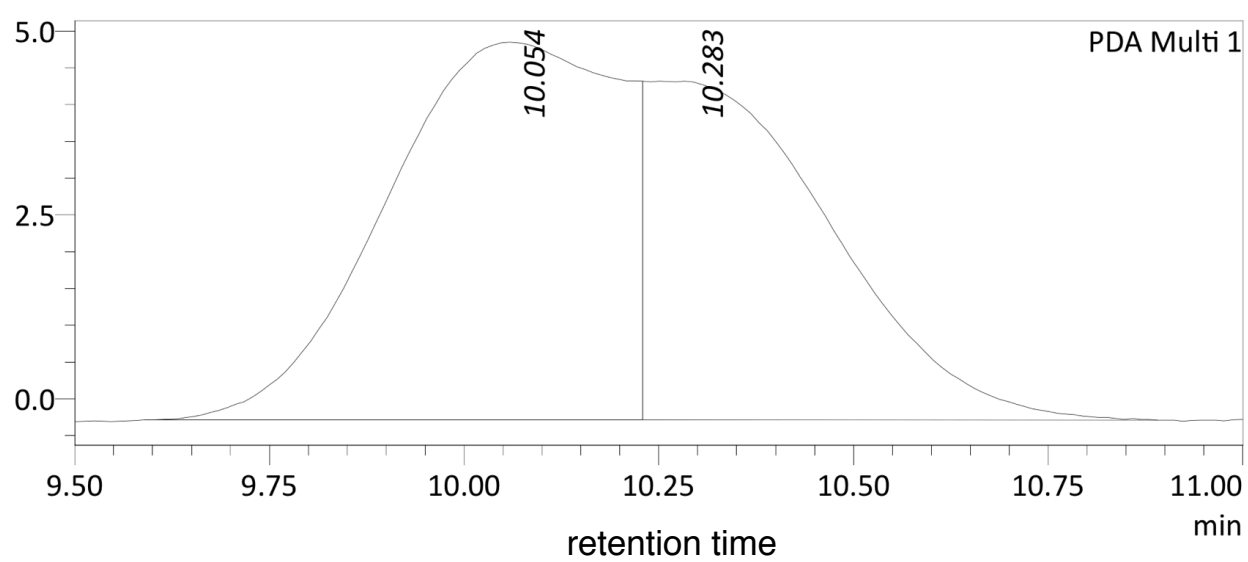

Figure S2. HPLC analysis of (a) the racemic mixture of QH-B, (b) (+)-QH-B, and (c) the thermal racemization progress of (+)-QH-B after heating over $185^{\circ} \mathrm{C}$ in 1,2,4-trichlorobenzene for $22 \mathrm{~h}$. 


\section{X-ray Crystallography and Structural Analysis}

Recrystallization of racemic mixture of QH-A by evaporating $n$-pentane solution yielded yellow crystals suitable for X-ray crystal structure analysis, and recrystallization of racemic mixture of QH-B from chloroform/2-propanol in vapor diffusion method yielded yellow crystals suitable for X-ray crystal structure analysis. Details of the crystal data and a summary of the intensity data collection parameters are listed in Table S1. A suitable crystal was mounted with mineral oil on a glass fiber and transferred to the goniometer of a Rigaku PILATUS diffractometer. Graphite-monochromated Mo K $\alpha$ radiation $(\lambda=0.71075 \AA)$ was used. The structures were solved by direct methods with (SIR-97) ${ }^{\mathrm{S} 2}$ and refined by full-matrix least-squares techniques against $F^{2}$ (SHELXL-97). ${ }^{\text {S3 }}$ The intensities were corrected for Lorentz and polarization effects. The non-hydrogen atoms were refined anisotropically. Hydrogen atoms were placed using AFIX instructions.

Table S1. Crystallographic data and structure refinement details for QH-A and QH-B.

\begin{tabular}{lll}
\hline & QH-A & QH-B \\
\hline formula & $\mathrm{C}_{132} \mathrm{H}_{112} \mathrm{~S}_{8}$ & $\mathrm{C}_{66} \mathrm{H}_{56} \mathrm{~S}_{4}$ \\
$\mathrm{fw}$ & 1954.70 & 977.35 \\
$T(\mathrm{~K})$ & $123(2)$ & $123(2)$ \\
$\lambda(\AA)$ & 0.71075 & 0.71075 \\
cryst syst & Triclinic & Monoclinic \\
space group & $P-1$ & $P 2_{1} / n$ \\
$a(\AA)$ & $16.2172(4)$ & $17.2313(19)$ \\
$b(\AA)$ & $17.2657(8)$ & $12.0935(11)$ \\
$c(\AA)$ & $21.5757(10)$ & $25.119(3)$ \\
$\alpha\left(^{\circ}\right)$ & $85.709(4)^{\circ}$ & $90^{\circ}$ \\
$\beta\left(^{\circ}\right)$ & $68.480(3)^{\circ}$ & $106.951(2)^{\circ}$ \\
$\gamma\left({ }^{\circ}\right)$ & $64.959(3)^{\circ}$ & $90^{\circ}$ \\
$V\left(\AA^{3}\right)$ & $5068.6(4)$ & $5007.1(9) \AA^{3}$ \\
$Z$ & 2 & 4 \\
$\left.D_{\text {calc }}(\mathrm{g} / \mathrm{cm})^{3}\right)$ & 1.281 & 1.297 \\
$\mu\left(\mathrm{mm}{ }^{-1}\right)$ & 0.231 & 0.233 \\
$\mathrm{~F}(000)$ & 2064 & 2064 \\
cryst size $(\mathrm{mm})$ & $0.15 \times 0.15 \times 0.10$ & $0.20 \times 0.05 \times 0.05$ \\
$\theta$ range $\left({ }^{\circ}\right)$ & $3.02-25.00^{\circ}$ & $3.05-25.00^{\circ}$ \\
reflns collected & 144604 & 67832 \\
indep reflns $/ R_{\text {int }}$ & $17822 / 0.0285$ & $8788 / 0.0438$ \\
params & 1314 & 680 \\
$\mathrm{GOF}$ on $F^{2}$ & 1.036 & 1.058 \\
$R_{1}, \mathrm{w} R_{2}[I>2 \sigma(I)]$ & $0.0358,0.0903$ & $0.0472,0.1124$ \\
$R_{1}, \mathrm{w} R_{2}($ all data $)$ & $0.0414,0.0954$ & $0.0676,0.1275$ \\
\hline
\end{tabular}




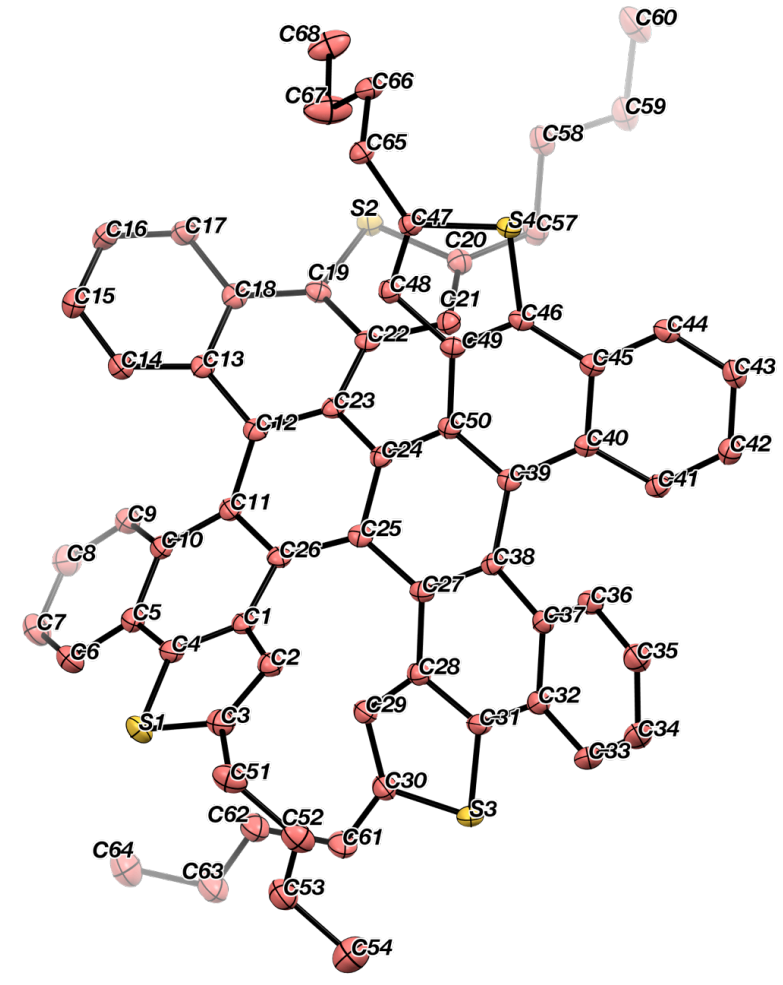

Splay angles of dithia[6]helicene substructures

$\mathrm{C} 1-\mathrm{C} 26-\mathrm{C} 27-\mathrm{C} 28=49.71(14)^{\circ}$

$\mathrm{C} 22-\mathrm{C} 23-\mathrm{C} 50-\mathrm{C} 49=53.18(14)^{\circ}$

Splay angles of [5]helicene substructures

$\mathrm{C} 10-\mathrm{C} 11-\mathrm{C} 12-\mathrm{C} 13=27.2(3)^{\circ}$

$\mathrm{C} 37-\mathrm{C} 38-\mathrm{C} 39-\mathrm{C} 40=20.2(3)^{\circ}$

Twist angles of each blades against naphthalene core

$\mathrm{C} 1-\mathrm{C} 26-\mathrm{C} 11-\mathrm{C} 10=28.9(2)^{\circ}$

$\mathrm{C} 13-\mathrm{C} 12-\mathrm{C} 23-\mathrm{C} 22=29.5(2)^{\circ}$

$\mathrm{C} 28-\mathrm{C} 27-\mathrm{C} 38-\mathrm{C} 37=27.0(2)^{\circ}$

C40-C39-C50-C49 = 28.9(2) ${ }^{\circ}$

Splay angles of dithia[6]helicene substructures

C71-C70-C119-C118 $=45.33(14)^{\circ}$

C92-C93-C96-C97 $=46.62(14)^{\circ}$

Splay angles of [5]helicene substructures

$\mathrm{C} 80-\mathrm{C} 81-\mathrm{C} 82-\mathrm{C} 83=29.7(3)^{\circ}$

C106-C107-C108-C109 = 29.3(3) ${ }^{\circ}$

Twist angles of each blades against naphthalene core

$\mathrm{C} 71-\mathrm{C} 70-\mathrm{C} 81-\mathrm{C} 80=28.3(2)^{\circ}$

$\mathrm{C} 83-\mathrm{C} 82-\mathrm{C} 93-\mathrm{C} 92=32.4(2)^{\circ}$

C97-C96-C107-C106 $=28.9(2)^{\circ}$

C109-C108-C119-C118 = 32.0(2) ${ }^{\circ}$

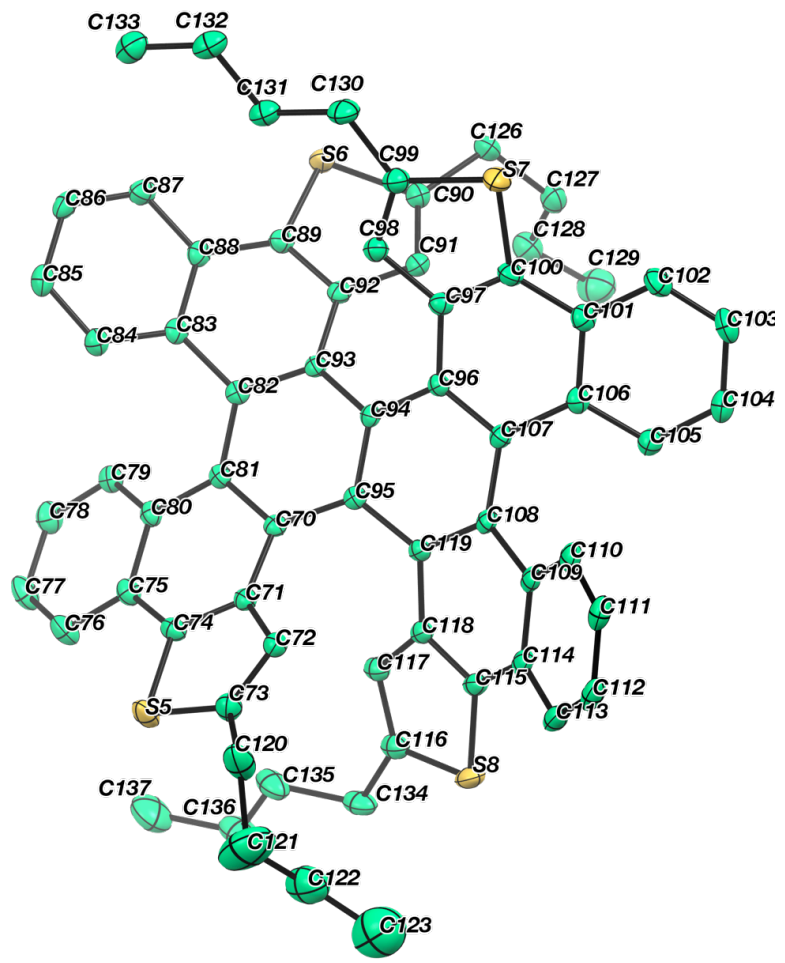

Figure S3. ORTEP drawing of QH-A-1 $((P, P)-(P, P)$, top) and QH-A-2 $((P, P)-(P, P)$, bottom $)$ with $50 \%$ probability. All hydrogen atoms and the minor part of the disordered moieties are omitted for clarity. Selected dihedral angles are also listed. 


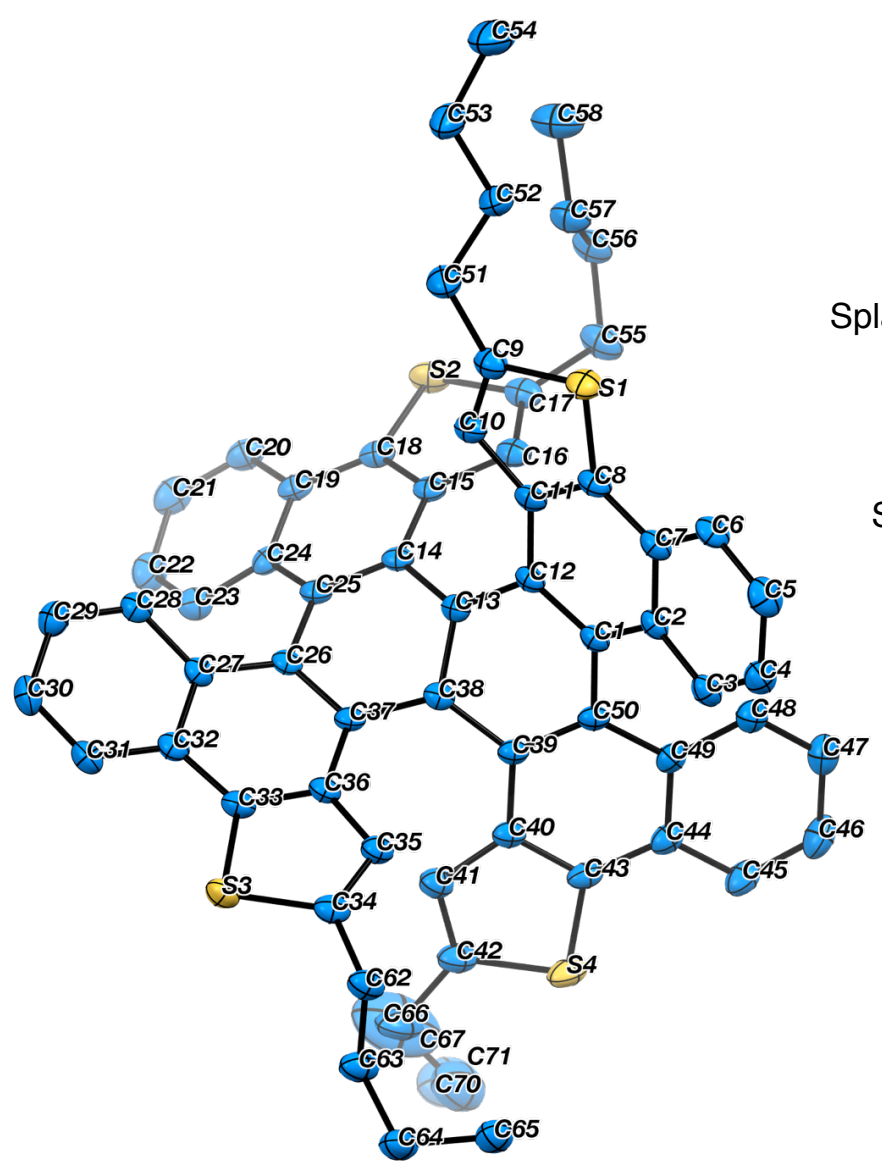

Splay angles of dithia[6]helicene substructures

$\mathrm{C} 11-\mathrm{C} 12-\mathrm{C} 14-\mathrm{C} 15=64.0(2)^{\circ}$

$\mathrm{C} 36-\mathrm{C} 37-\mathrm{C} 39-\mathrm{C} 40=61.2(2)^{\circ}$

Splay angles of [5]helicene substructures

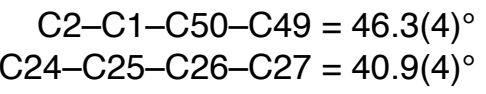

Twist angles of each blades against naphthalene core

$\mathrm{C} 2-\mathrm{C} 1-\mathrm{C} 12-\mathrm{C} 11=8.6(4)^{\circ}$

$\mathrm{C} 15-\mathrm{C} 14-\mathrm{C} 25-\mathrm{C} 24=4.6(4)^{\circ}$

$\mathrm{C} 27-\mathrm{C} 26-\mathrm{C} 37-\mathrm{C} 36=3.8(4)^{\circ}$

$\mathrm{C} 40-\mathrm{C} 39-\mathrm{C} 50-\mathrm{C} 49=9.0(4)^{\circ}$

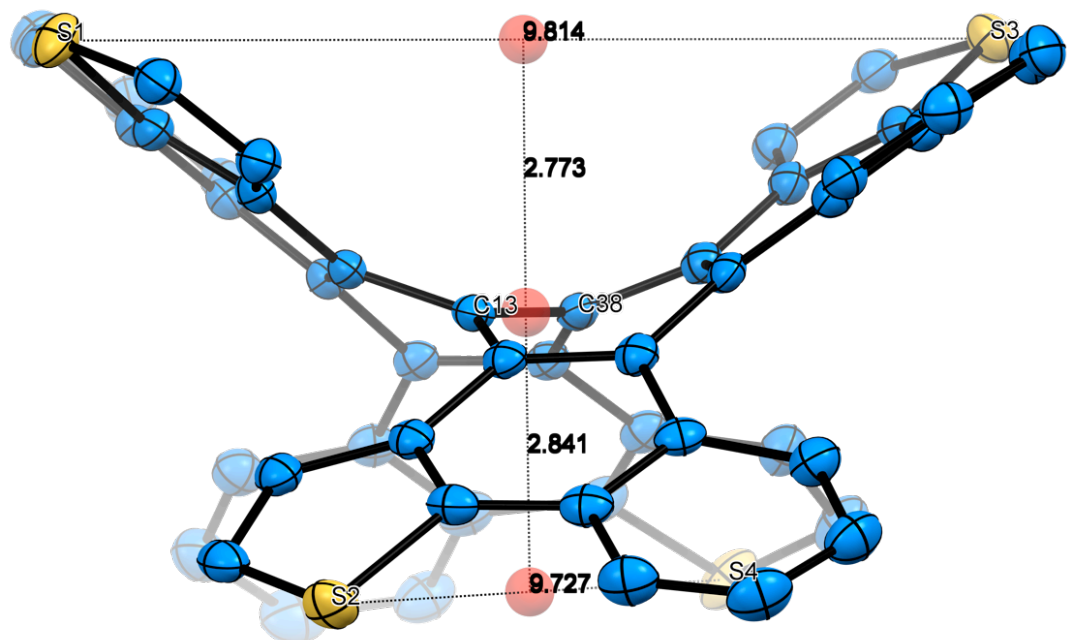

Figure S4. ORTEP drawing of QH-B with 50\% probability. All hydrogen atoms and the minor part of the disordered moieties are omitted for clarity. Selected dihedral angles are also listed. Depth of double concave structures was estimated by using the distance from the centroids of sulfur atoms at the diagonal positions to the centroid of the bond C13-C38 in the naphthalene core (centroids are colored in red). 


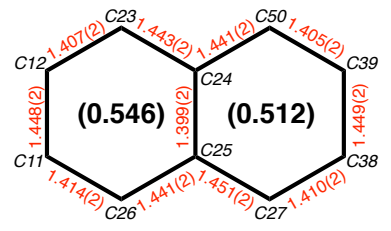

QH-A-1

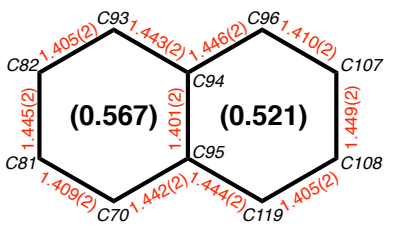

QH-A-2

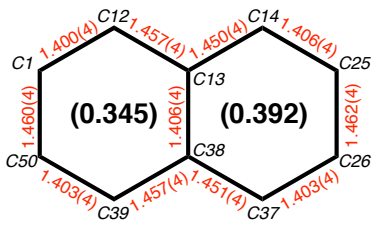

QH-B

Figure S5. Bond lengths and values of HOMA of naphthalene cores in X-ray structures.

Table S2. Comparison of dihedral angles of the X-ray structures and the optimized structures of QH-A and QH-B.

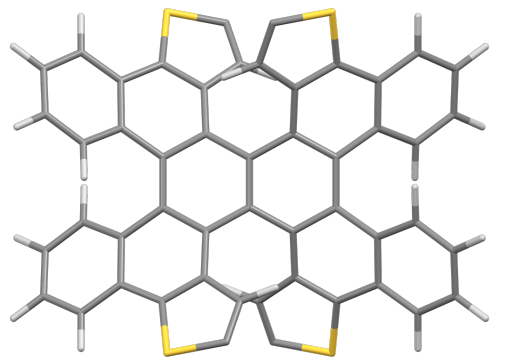

QH-A

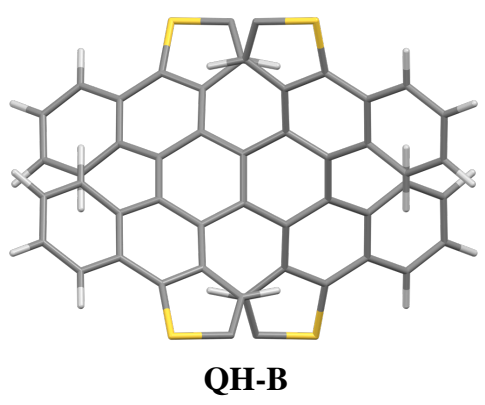

QH-B

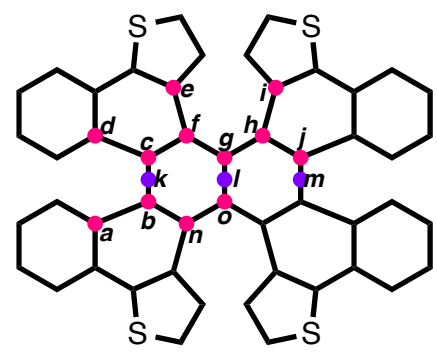

\begin{tabular}{|c|c|c|c|c|c|}
\hline & \multicolumn{3}{|c|}{ QH-A } & \multicolumn{2}{|c|}{ QH-B } \\
\hline & X-ray (QH-A-1) & X-ray (QH-A-2) & optimized $^{a}$ & $\mathrm{X}$-ray & optimized $^{a}$ \\
\hline \multicolumn{6}{|l|}{ twist angles } \\
\hline $\mathrm{C}(\mathrm{c})-\mathrm{C}(\mathrm{k})-\mathrm{C}(\mathrm{m})-\mathrm{C}(\mathrm{j})$ & $13.9^{\circ}$ & $6.5^{\circ}$ & $8.9^{\circ}$ & $69.5^{\circ}$ & $70.2^{\circ}$ \\
\hline $\mathrm{C}(\mathrm{c})-\mathrm{C}(\mathrm{k})-\mathrm{C}(\mathrm{l})-\mathrm{C}(\mathrm{g})$ & $8.1^{\circ} / 5.8^{\circ}$ & $3.7^{\circ} / 2.8^{\circ}$ & $4.4^{\circ}$ & $35.3^{\circ} / 34.2^{\circ}$ & $35.1^{\circ}$ \\
\hline \multicolumn{6}{|l|}{ splay angles } \\
\hline$C(a)-C(b)-C(c)-C(d)$ & $27.2^{\circ} / 20.2^{\circ}$ & $29.7^{\circ} / 29.3^{\circ}$ & $26.1^{\circ}$ & $46.3^{\circ} / 40.9^{\circ}$ & $42.3^{\circ}$ \\
\hline$C(e)-C(f)-C(h)-C(i)$ & $49.7^{\circ} / 53.2^{\circ}$ & $45.3^{\circ} / 46.6^{\circ}$ & $49.5^{\circ}$ & $64.0^{\circ} / 61.2^{\circ}$ & $61.9^{\circ}$ \\
\hline \multicolumn{6}{|l|}{$\begin{array}{l}\text { dihedral angles } \\
\text { of naphthalene core }\end{array}$} \\
\hline$C(f)-C(c)-C(b)-C(n)$ & $11.3^{\circ} / 8.4^{\circ}$ & $14.8^{\circ} / 13.4^{\circ}$ & $13.8^{\circ}$ & $35.6^{\circ} / 32.5^{\circ}$ & $33.6^{\circ}$ \\
\hline $\mathrm{C}(\mathrm{f})-\mathrm{C}(\mathrm{g})-\mathrm{C}(\mathrm{l})-\mathrm{C}(\mathrm{o})$ & $23.9^{\circ} / 26.0^{\circ}$ & $20.6^{\circ} / 21.3^{\circ}$ & $23.3^{\circ}$ & $42.7^{\circ} / 42.5^{\circ}$ & $43.6^{\circ}$ \\
\hline
\end{tabular}

[a] B3LYP/6-31G(d) 


\section{Photophysical Study}

UV/Vis absorption spectra were recorded on a Shimadzu UV-3510 spectrometer with a resolution of $0.5 \mathrm{~nm}$. Emission spectra were measured with an FP-6600 Hitachi spectrometer with a resolution of $0.2 \mathrm{~nm}$ upon excitation at $381 \mathrm{~nm}$ for QH-A and $405 \mathrm{~nm}$ for QH-B. Circular dichroism spectra were measured with a JASCO FT/IR6100. Dilute solutions in degassed spectral grade dichloromethane in a $1 \mathrm{~cm}$ square quartz cell were used for measurements. Absolute fluorescence quantum yields were determined with a Hamamatsu C9920-02 calibrated integrating sphere system upon excitation at 400 $\mathrm{nm}$ for QH-A or at $420 \mathrm{~nm}$ for QH-B.

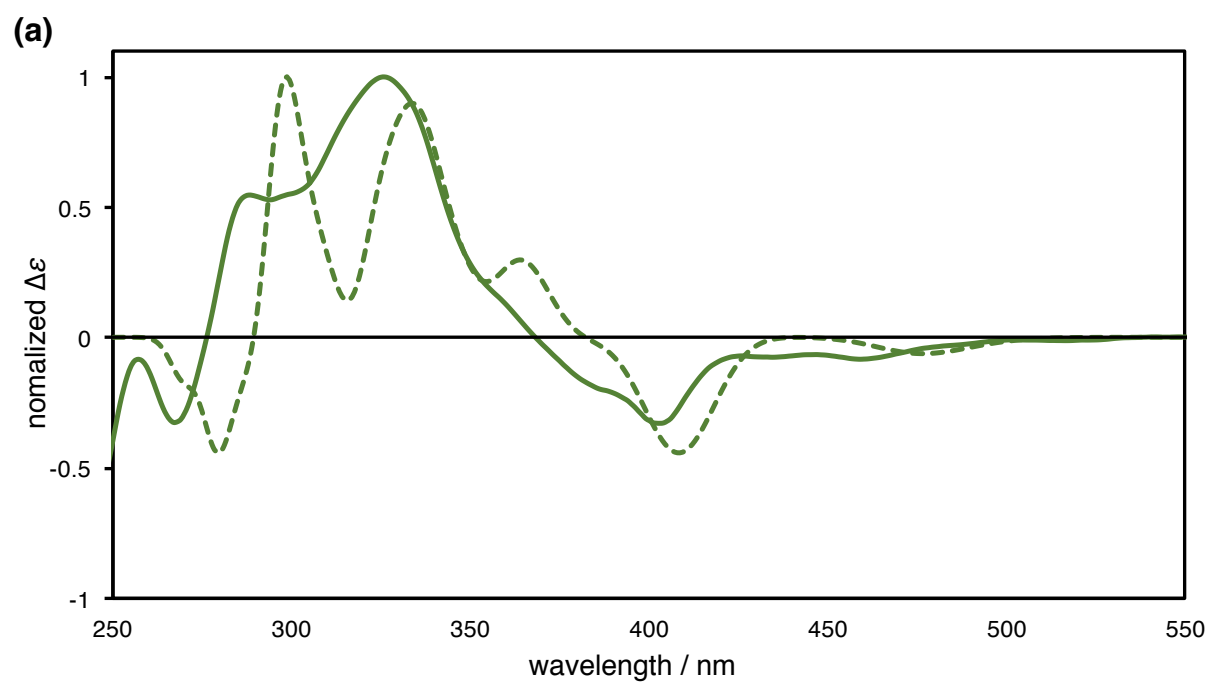

(b)

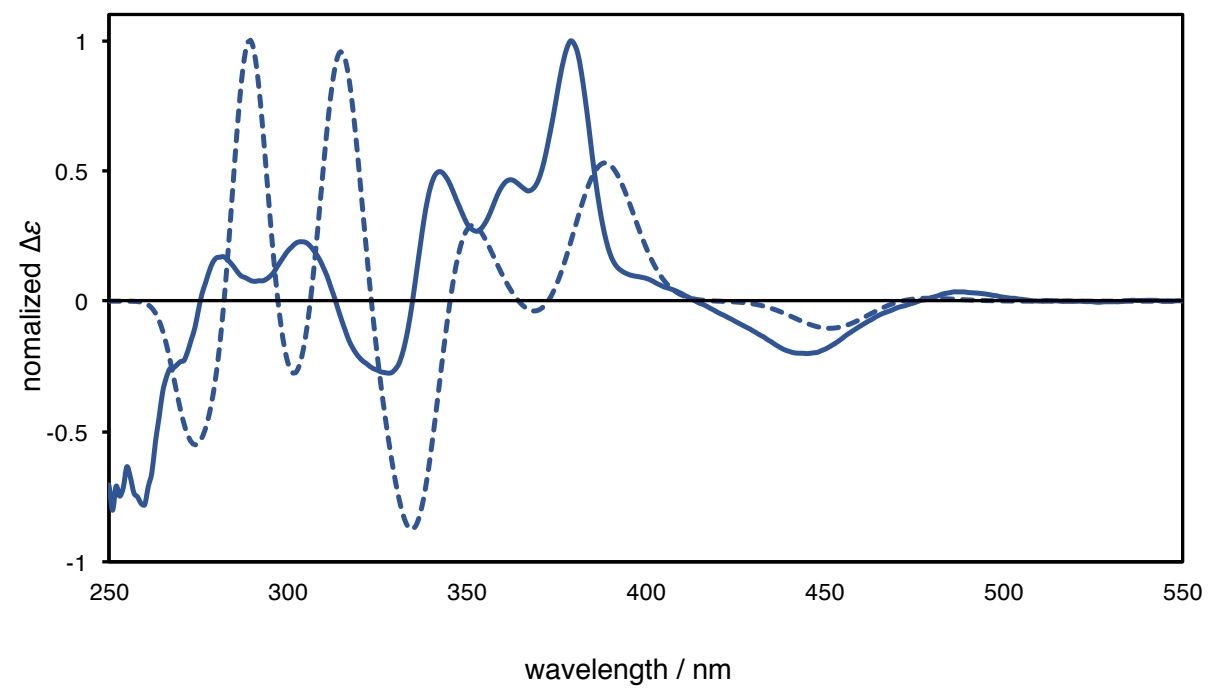

Figure S6. Circular dichloism spectra of (a) QH-A and (b) QH-B. Experimental spectra are solid lines, and simulated spectra (B3LYP/6-31G(d) level) are broken lines. 


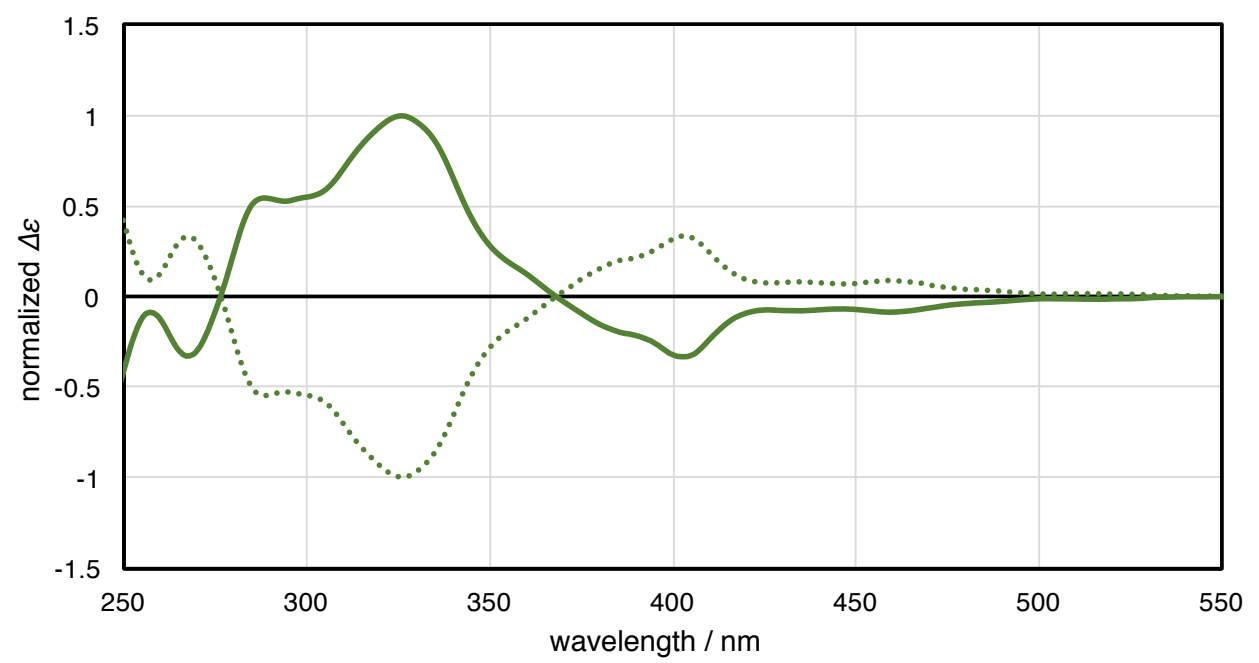

Figure S7. Circular dichloism spectra of (+)-QH-A (solid line) and (-)-QH-A (dot line). 


\section{Computational Study}

The Gaussian 09 program $^{\text {s4 }}$ running on a SGI Altix4700 system was used for optimization (B3LYP/6-31G(d)). ${ }^{\text {S5 }}$ All structures were optimized without any symmetry assumptions. Zero-point energy, enthalpy, and Gibbs free energy at $298.15 \mathrm{~K}$ and $1 \mathrm{~atm}$ were estimated from the gas-phase studies unless otherwise noted. Harmonic vibration frequency calculations at the same level were performed to verify all stationary points as local minima (with no imaginary frequency) or transition states (with one imaginary frequency). All NMR study was performed at B3LYP/6-311+G(2d,p)//B3LYP/6-31G(d) level. Tetramethylsilane $\left(\mathrm{SiMe}_{4}\right)$ was used as reference $(\delta$ $0.00 \mathrm{ppm}$ ) for the estimation of ${ }^{1} \mathrm{H}$ NMR chemical shifts. Visualization of the results was performed by use of GaussView 5.0 software.

Table S3. Uncorrected and thermal-corrected (298 K) energies of stationary points (Hartree). ${ }^{\mathrm{a}}$

\begin{tabular}{|c|c|c|c|c|}
\hline structure & $E$ & $E+Z P E$ & $H$ & $G$ \\
\hline QH-A & -3669.85187963 & -3669.165165 & -3669.118799 & -3669.241538 \\
\hline QH-B & -3669.86422209 & -3669.177467 & -3669.131027 & -3669.254435 \\
\hline QH-C & -3669.85486381 & -3669.168189 & -3669.121772 & -3669.245131 \\
\hline QH-D & -3669.84831139 & -3669.161862 & -3669.115471 & -3669.238444 \\
\hline QH-E & -3669.84143375 & -3669.154818 & -3669.108493 & -3669.231471 \\
\hline $\mathbf{T S}_{\mathrm{A}-\mathrm{C}}$ & -3669.80498465 & -3669.119266 & -3669.073412 & -3669.195353 \\
\hline $\mathbf{T S}_{\mathrm{A}-\mathrm{D}}$ & -3669.77916720 & -3669.093652 & -3669.047956 & -3669.169088 \\
\hline $\mathbf{T S}_{\mathrm{B}-\mathrm{C}}$ & -3669.81404938 & -3669.128210 & -3669.082306 & -3669.204508 \\
\hline $\mathbf{T S}_{\mathrm{B}-\mathrm{D}}$ & -3669.79518508 & -3669.109794 & -3669.063862 & -3669.186529 \\
\hline $\mathbf{T S} \mathbf{S}_{\mathrm{C}-\mathbf{E}}$ & -3669.80849210 & -3669.122642 & -3669.076938 & -3669.197818 \\
\hline $\mathbf{T S}_{\mathrm{D}-\mathrm{E}}$ & -3669.82961756 & -3669.143677 & -3669.097875 & -3669.219463 \\
\hline dithia[6]helicene & -1720.60918362 & -1720.285264 & -1720.263932 & -1720.333347 \\
\hline $\mathbf{T S}_{\text {dithia[6]helicene }}$ & -1720.54702510 & -1720.223952 & -1720.203311 & -1720.270761 \\
\hline
\end{tabular}

a) $E$ : electronic energy; $Z P E$ : zero-point energy; $H\left(=E+Z P E+E_{\text {vib }}+E_{\text {rot }}+E_{\text {trans }}+R T\right)$ : sum of electronic and thermal enthalpies; $G(=H-T S)$ : sum of electronic and thermal free energies.

Table S4. Cartesian coordinates of optimized species.

\section{QH-A}

\begin{tabular}{|c|c|c|c|c|c|c|c|c|}
\hline C -0.704821 & 0.000005 & -0.000001 & 3.715614 & -4.828809 & 0.082361 & H - 1.586974 & 6.668565 & -1.811694 \\
\hline C $\quad 0.704820$ & -0.000002 & -0.000004 & 3.242341 & -5.904238 & -0.642571 & H -0.144801 & 4.740719 & -1.400178 \\
\hline C 1.391464 & -1.249264 & 0.256786 & 1.946330 & -5.855278 & -1.187368 & 0.144847 & 4.740716 & 1.400180 \\
\hline C $\quad 0.721292$ & -2.480555 & 0.056044 & 1.128227 & -4.766308 & -0.948576 & 1.587038 & 6.668547 & 1.811699 \\
\hline C -0.721317 & -2.480548 & -0.056047 & C -3.386249 & 2.511149 & 0.951130 & 3.880122 & 6.765550 & 0.821238 \\
\hline C - 1.391477 & -1.249251 & -0.256791 & 3.386274 & 2.511118 & -0.951129 & 4.735810 & 4.830527 & -0.457499 \\
\hline C - 1.391464 & 1.249267 & 0.256790 & 3.386250 & -2.511148 & 0.951126 & H -0.144832 & -4.740722 & 1.400155 \\
\hline C -0.721293 & 2.480558 & 0.056044 & C -3.386275 & -2.511117 & -0.951127 & H - 1.587017 & -6.668560 & 1.811667 \\
\hline C $\quad 0.721317$ & 2.480551 & -0.056045 & C -2.701289 & 1.301567 & 0.883642 & H -3.880107 & -6.765561 & 0.821217 \\
\hline C 1.391476 & 1.249254 & -0.256793 & C -3.386667 & 0.266575 & 1.616664 & $\mathrm{H}-4.735804$ & -4.830532 & -0.457504 \\
\hline C -1.554918 & 3.671564 & -0.155582 & C -4.552427 & 0.668672 & 2.193282 & 4.735756 & -4.830578 & 0.457512 \\
\hline C 1.554953 & 3.671549 & 0.155583 & S-4.8732340 & 2.365653 & 1.862057 & 3.880040 & -6.765602 & -0.821204 \\
\hline C - 1.554949 & -3.671549 & 0.155576 & S-4.8732610 & -2.365607 & -1.862050 & 1.586953 & -6.668581 & -1.811658 \\
\hline C 1.554913 & -3.671565 & -0.155574 & C -4.552441 & -0.668628 & -2.193274 & 0.144787 & -4.740728 & -1.400153 \\
\hline$C-2.906057$ & 3.691853 & 0.307338 & $C-3.386676$ & -0.266541 & -1.616658 & H -3.007457 & -0.739650 & 1.737272 \\
\hline
\end{tabular}




\begin{tabular}{|c|c|c|c|c|c|c|c|c|}
\hline C -3.715622 & 4.828803 & 0.082349 & C -2.701304 & -1.301540 & -0.883639 & H -3.007458 & 0.739681 & -1.737266 \\
\hline C -3.242354 & 5.904227 & -0.642593 & S 4.8732590 & 2.365609 & -1.8620560 & 3.007453 & -0.739677 & -1.737275 \\
\hline C -1.946346 & 5.855266 & -1.187395 & C 4.552436 & 0.668631 & -2.193283 & 3.007466 & 0.739657 & 1.737252 \\
\hline C -1.128238 & 4.766301 & -0.948597 & 3.386672 & 0.266544 & -1.616665 & H -5.111874 & -1.156498 & 3.145115 \\
\hline C 1.128284 & 4.766289 & 0.948599 & C $\quad 2.701302$ & 1.301542 & -0.883643 & H -5.624563 & 0.290332 & 4.032971 \\
\hline C $\quad 1.946402$ & 5.855246 & 1.187399 & 2.701291 & -1.301564 & 0.883634 & H -6.491543 & -0.204985 & 2.573570 \\
\hline C $\quad 3.242411$ & 5.904196 & 0.642598 & 3.386673 & -0.266569 & 1.616649 & H -5.624579 & -0.290276 & -4.032958 \\
\hline C 3.715669 & 4.828767 & -0.082345 & C 4.552434 & -0.668665 & 2.193267 & H -6.491551 & 0.205046 & -2.573555 \\
\hline C 2.906093 & 3.691826 & -0.307336 & S 4.8732370 & -2.365649 & 1.862050 & H -5.111876 & 1.156548 & -3.145103 \\
\hline C -1.128273 & -4.766294 & 0.948580 & C -5.496733 & -0.138500 & 3.031364 & H 5.624569 & 0.290283 & -4.032972 \\
\hline C -1.946386 & -5.855255 & 1.187375 & C -5.496743 & 0.138553 & -3.031351 & 6.491544 & -0.205044 & -2.573571 \\
\hline C -3.242398 & -5.904203 & 0.642580 & C 5.496734 & -0.138549 & -3.031365 & 5.111866 & -1.156543 & -3.145118 \\
\hline C -3.715662 & -4.828771 & -0.082354 & C $\quad 5.496743$ & 0.138509 & 3.031342 & 5.111887 & 1.156508 & 3.145089 \\
\hline C -2.906090 & -3.691826 & -0.307341 & H -4.735763 & 4.830572 & 0.457503 & 5.624574 & -0.290318 & 4.032951 \\
\hline C 2.906053 & -3.691855 & 0.307343 & H -3.880058 & 6.765588 & -0.821232 & 6.491552 & 0.204990 & 2.573546 \\
\hline
\end{tabular}

\section{QH-B}

\begin{tabular}{|c|c|c|c|c|c|c|c|c|}
\hline$C-0.000014$ & -0.704075 & -0.000002 & C 4.675623 & 2.653154 & 2.6786600 & H -6.606745 & 2.130690 & -3.441912 \\
\hline C -0.000026 & 0.704092 & 0.000005 & 5.756864 & 1.814987 & 2.8431240 & H - 4.663457 & 3.624270 & -3.166310 \\
\hline C $\quad 1.217927$ & 1.328831 & 0.4871940 & 5.733403 & 0.529250 & 2.2656690 & H -4.663361 & -3.624349 & 3.166340 \\
\hline 2.426510 & 0.598266 & 0.4195900 & C 4.664640 & 0.134487 & 1.4879080 & H -6.606651 & -2.130784 & 3.442012 \\
\hline 2.426522 & -0.598209 & -0.419577 & C -2.346733 & -3.013249 & 1.870371 & H - 6.555017 & 0.159987 & 2.439983 \\
\hline 1.217951 & -1.328791 & -0.487186 & C - -2.346792 & 3.013199 & -1.870394 & H - 4.660008 & 0.863052 & 1.067901 \\
\hline C -1.217964 & -1.328813 & 0.487189 & C $\quad 2.346726$ & 3.013263 & 1.8703620 & H 4.660050 & 0.863054 & -1.067674 \\
\hline C -2.426545 & -0.598241 & 0.419611 & C 2.346773 & -3.013209 & -1.870350 & H $\quad 6.555140$ & 0.159984 & -2.439648 \\
\hline C -2.426560 & 0.598218 & -0.419583 & C - 1.178241 & -2.552421 & 1.260168 & Н $\quad 6.606802$ & -2.130768 & -3.441718 \\
\hline C - 1.217989 & 1.328804 & -0.487188 & C -0.051887 & -3.395572 & 1.575489 & 4.663477 & -3.624314 & -3.166193 \\
\hline C -3.562174 & -0.995051 & 1.236025 & C -0.358467 & -4.474080 & 2.346657 & 4.663408 & 3.624381 & 3.1662390 \\
\hline C -3.562210 & 0.995007 & -1.235981 & S - 2.066786 & -4.501578 & 2.755608 & 6.606741 & 2.130848 & 3.4417820 \\
\hline C 3.562195 & -0.995024 & -1.235925 & S $\quad 2.066857$ & -4.501522 & -2.755625 & 6.555110 & -0.159900 & 2.439703 \\
\hline 3.562170 & 0.995090 & 1.2359480 & 0.358521 & -4.474028 & -2.346737 & 4.660040 & -0.862980 & 1.067703 \\
\hline-4.664644 & 0.134373 & -1.487992 & 0.051915 & -3.395534 & -1.575560 & Н $\quad 0.958749$ & -3.203844 & 1.243057 \\
\hline-5.733394 & 0.529108 & -2.265785 & C $\quad 1.178258$ & -2.552389 & -1.260184 & H - 0.958733 & -3.203812 & -1.243160 \\
\hline C -5.756878 & 1.814849 & -2.843229 & C $\quad 1.178217$ & 2.552430 & 1.2601910 & H - 0.958779 & 3.203834 & 1.243167 \\
\hline C -4.675663 & 2.653042 & -2.678731 & C $\quad 0.051867$ & 3.395567 & 1.5755640 & H $\quad 0.958703$ & 3.203817 & -1.243158 \\
\hline C -3.551439 & 2.253429 & -1.913922 & C $\quad 0.358463$ & 4.474068 & 2.3467340 & H $\quad 1.581957$ & -5.347417 & 2.499448 \\
\hline C -3.551381 & -2.253484 & 1.913945 & S 2.066796 & 4.5015800 & 2.755625 & 0.568445 & -5.618714 & 3.928804 \\
\hline C -4.675584 & -2.653114 & 2.678776 & S - 2.066867 & 4.501500 & -2.755686 & H $\quad 0.271785$ & -6.538577 & 2.448550 \\
\hline$C-5.756800$ & -1.814930 & 2.843312 & C -0.358538 & 4.474017 & -2.346770 & H - 0.568337 & -5.618624 & -3.928942 \\
\hline C -5.733338 & -0.529180 & 2.265887 & C - -0.051941 & 3.395533 & -1.575575 & H -0.271727 & -6.538522 & -2.448700 \\
\hline C -4.664608 & -0.134426 & 1.488075 & C - 1.178287 & 2.552391 & -1.260201 & H - 1.581897 & -5.347360 & -2.499615 \\
\hline C $\quad 4.664661$ & -0.134414 & -1.487875 & C $\quad 0.560543$ & -5.552709 & 2.833871 & H $\quad 0.568340$ & 5.618596 & -3.928976 \\
\hline C 5.733436 & -0.529171 & -2.265622 & C -0.560472 & -5.552645 & -2.834008 & H $\quad 0.271708$ & 6.538510 & -2.448749 \\
\hline C $\quad 5.756915$ & -1.814910 & -2.843072 & C $\quad 0.560460$ & 5.552629 & -2.834041 & H $\quad 1.581881$ & 5.347351 & -2.499631 \\
\hline C 4.675677 & -2.653084 & -2.678619 & C -0.560541 & 5.552680 & 2.833995 & H - 1.581965 & 5.347382 & 2.499605 \\
\hline C 3.551430 & -2.253451 & -1.913855 & H -4.660029 & -0.863100 & -1.067804 & H -0.568405 & 5.618671 & 3.928928 \\
\hline 3.551388 & 2.253516 & 1.9138790 & H -6.555073 & -0.160067 & -2.439852 & H -0.271808 & 6.538556 & 2.448677 \\
\hline
\end{tabular}




\begin{tabular}{|c|c|c|c|c|c|c|c|c|}
\hline 0.700225 & -0.031880 & -0.074179 & C -3.630603 & 4.774952 & 0.881877 & H - 2.480738 & -6.706379 & -3.093100 \\
\hline C -0.700250 & -0.031992 & 0.074172 & C -3.306468 & 5.879762 & 0.120023 & H - -3.963110 & -4.789424 & -2.635210 \\
\hline-1.331353 & 1.202907 & 0.458477 & C - -2.149904 & 5.852021 & -0.679500 & H $\quad 3.963907$ & -4.788735 & 2.635205 \\
\hline C -0.698453 & 2.444916 & 0.188263 & C -1.305926 & 4.756785 & -0.649369 & 2.481878 & -6.705944 & 3.093128 \\
\hline C $\quad 0.698043$ & 2.445024 & -0.188299 & C $\quad 3.216407$ & -2.435256 & 1.475376 & 0.074084 & -6.592291 & 2.425854 \\
\hline C $\quad 1.331148$ & 1.203111 & -0.458481 & C -3.216030 & -2.435810 & -1.475382 & H - 0.770481 & -4.667061 & 1.181676 \\
\hline C 1.386080 & -1.261675 & 0.328421 & C -3.177054 & 2.412133 & 1.539931 & H $\quad 0.434244$ & 4.750235 & 1.291744 \\
\hline C $\quad 0.644952$ & -2.462473 & 0.344625 & C $\quad 3.176688$ & 2.412610 & -1.539905 & 1.919841 & 6.687028 & 1.33609 \\
\hline C -0.644584 & -2.462577 & -0.344602 & C 2.692826 & -1.243967 & 0.961726 & 3.960491 & 6.747077 & -0.108151 \\
\hline C -1.385910 & -1.261901 & -0.328421 & C $\quad 3.575525$ & -0.135285 & 1.236522 & 4.555529 & 4.759705 & -1.451382 \\
\hline C 1.118236 & -3.610687 & 1.099635 & C 4.734384 & -0.475797 & 1.862918 & H - 4.556290 & 4.758996 & 1.451380 \\
\hline C -1.117665 & -3.610876 & -1.099608 & 4.802514 & -2.198973 & 2.185475 & Н -3.961625 & 6.746428 & 0.108075 \\
\hline C $\quad 1.563391$ & 3.634738 & -0.176650 & 4.514652 & 2.189316 & -2.6454990 & H - 1.921003 & 6.686678 & -1.336217 \\
\hline C -1.563997 & 3.634490 & 0.176602 & C 4.192938 & 0.462659 & -2.754903 & H - -0.435085 & 4.750124 & -1.291855 \\
\hline C -0.274525 & -4.697296 & -1.459079 & C 3.130980 & 0.109953 & -1.978753 & H $\quad 3.357691$ & 0.892864 & 0.99195 \\
\hline C -0.749447 & -5.783351 & -2.164356 & C $\quad 2.532342$ & 1.210548 & -1.269319 & H $\quad 2.764451$ & -0.907777 & -1.928075 \\
\hline C -2.103232 & -5.841784 & -2.554139 & C -2.532525 & 1.210167 & 1.269352 & H - 2.764278 & -0.908179 & 1.928164 \\
\hline C - -2.933915 & -4.775799 & -2.286608 & C -3.130966 & 0.109494 & & H -3.357874 & 0.892299 & -0.992078 \\
\hline C -2.454887 & -3.632636 & -1.598440 & C - 4.192959 & 0.462048 & 2.755001 & H $\quad 5.622302$ & 1.458839 & 2.002999 \\
\hline C 2.455469 & -3.632212 & 1.598452 & S - 4.514945 & 2.188652 & 2.645574 & H $\quad 6.059146$ & 0.380128 & 3.341578 \\
\hline C 2.934704 & -4.775289 & 2.286619 & S - 4.802173 & -2.199821 & -2.185500 & H 6.796792 & 0.157353 & $1.75040 \mathrm{c}$ \\
\hline C 2.104211 & -5.841417 & 2.554171 & C -4.734331 & -0.476623 & -1.862995 & H 4.984791 & -0.098212 & -4.687079 \\
\hline C $\quad 0.750412$ & -5.783220 & 2.164407 & C -3.575529 & -0.135897 & -1.236606 & H 6.083330 & -0.408263 & -3.336752 \\
\hline C $\quad 0.275292$ & -4.697253 & 1.459127 & C - -2.692650 & -1.244422 & -0.961753 & H 4.668702 & -1.446041 & -3.580129 \\
\hline C 1.305103 & 4.757016 & 0.649279 & C $\quad 5.863988$ & 0.423837 & 2.262960 & H - 6.059215 & 0.379040 & -3.341694 \\
\hline C $\quad 2.148900$ & 5.852391 & 0.679404 & C 5.028313 & -0.414172 & -3.637451 & Н -6.796849 & 0.156172 & -1.750529 \\
\hline C 3.305482 & 5.880299 & -0.120088 & C -5.864083 & 0.422807 & -2.263073 & H -5.622580 & 1.457857 & -2.003131 \\
\hline C 3.629825 & 4.775522 & -0.881903 & C -5.028168 & -0.414897 & 3.637593 & H -4.668395 & -1.446710 & 3.580282 \\
\hline C $\quad 2.800478$ & 3.630400 & -0.893325 & H $\quad 0.771240$ & -4.666921 & -1.181614 & Н -4.984664 & -0.098908 & 4.687213 \\
\hline C - 2.801064 & 3.629968 & 0.893308 & H - 0.072975 & -6.592307 & -2.425785 & H -6.083196 & -0.409162 & 3.336927 \\
\hline
\end{tabular}

\section{QH-D}

\begin{tabular}{|c|c|c|c|c|c|c|c|c|}
\hline C -0.723118 & -0.000116 & -0.000092 & 3.410378 & -4.640299 & -1.584635 & 3.263347 & 6.231699 & 3.0137160 \\
\hline 0.691809 & 0.000047 & -0.000090 & 2.727402 & -5.472380 & -2.451504 & 4.486653 & 4.733849 & 1.4738810 \\
\hline 1.364815 & -1.282737 & 0.037229 & 1.339733 & -5.313258 & -2.628351 & H -4.561761 & 4.783072 & -1.587750 \\
\hline 0.610412 & -2.437581 & -0.287031 & 0.650580 & -4.356227 & -1.906293 & H -3.434452 & 6.979152 & -1.495092 \\
\hline C -0.778052 & -2.466103 & 0.140792 & C -3.386876 & 2.371390 & -1.247746 & H -0.981329 & 7.069438 & -0.997954 \\
\hline$C-1.410695$ & -1.219087 & 0.370781 & 3.375581 & 2.695587 & -0.000684 & 0.232703 & 5.053412 & -0.429349 \\
\hline C - 1.410992 & 1.218707 & -0.370911 & 3.376214 & -2.694856 & 0.000672 & 0.233901 & -5.053393 & 0.429588 \\
\hline C -0.778635 & 2.465854 & -0.140835 & C -3.386299 & -2.372140 & 1.247746 & H - 0.979646 & -7.069643 & 0.998420 \\
\hline 0.609838 & 2.437628 & 0.2869720 & C -2.689384 & 1.184687 & -1.046049 & H - 3.432786 & -6.979886 & 1.495571 \\
\hline 1.364519 & 1.282987 & -0.037362 & C -3.380384 & 0.059553 & -1.625095 & H -4.560612 & -4.784064 & 1.588011 \\
\hline C -1.460131 & 3.704708 & -0.541138 & C -4.574573 & 0.371695 & -2.197004 & 4.487793 & -4.732950 & -1.473743 \\
\hline 1.312339 & 3.509446 & 0.9851800 & S - 4.911872 & 2.092332 & -2.068710 & 3.264867 & -6.231188 & -3.013503 \\
\hline C -1.459253 & -3.705078 & 0.541234 & S -4.911345 & -2.093335 & 2.068704 & 0.807620 & -5.937691 & -3.340563 \\
\hline 1.313176 & -3.509275 & -0.985164 & C -4.574440 & -0.372605 & 2.196805 & H -0.417600 & -4.236624 & -2.052839 \\
\hline 0.649524 & 4.356184 & 1.9063470 & C -3.380332 & -0.060253 & 1.624843 & H - 2.992387 & -0.949944 & -1.628292 \\
\hline 1.338437 & 5.313335 & 2.6284750 & C -2.689082 & -1.185299 & 1.045929 & H - 2.992562 & 0.949337 & 1.627905 \\
\hline 2.726071 & 5.472797 & 2.4516620 & 2.745448 & -1.524078 & 0.415524 & 3.251077 & 0.147617 & 1.8016890 \\
\hline
\end{tabular}




\begin{tabular}{|c|c|c|c|c|c|c|c|c|}
\hline 3.409259 & 4.640933 & 1.5847530 & 3.569990 & -0.769352 & 1.322866 & 3.251146 & -0.146753 & -1.801950 \\
\hline 2.729901 & 3.634266 & 0.8631530 & 4.785426 & -1.332104 & 1.569815 & H -5.143193 & -1.564835 & -2.888364 \\
\hline 2.819855 & 3.653484 & -0.987740 & 4.984545 & -2.833950 & 0.6756320 & Н -5.742229 & -0.244272 & -3.909399 \\
\hline C - 3.518448 & 4.847525 & -1.290141 & 4.983885 & 2.8351300 & -0.675616 & H - 6.510224 & -0.575844 & -2.351632 \\
\hline C -2.887387 & 6.071904 & -1.254118 & 4.785136 & 1.333310 & -1.569924 & Н -6.510307 & 0.574505 & 2.351341 \\
\hline C -1.513964 & 6.123435 & -0.955689 & 3.569833 & 0.770244 & -1.323034 & Н -5.742224 & 0.243287 & 3.909139 \\
\hline C -0.826185 & 4.972922 & -0.620332 & 2.745101 & 1.524685 & -0.415627 & Н -5.143500 & 1.563872 & 2.887950 \\
\hline C -0.825004 & -4.973132 & 0.620567 & C -5.545662 & -0.547401 & -2.873593 & 6.102425 & 1.553824 & -3.270877 \\
\hline C - 1.512506 & -6.123772 & 0.956056 & C -5.545734 & 0.546344 & 2.873298 & H $\quad 6.800266$ & 0.639159 & -1.928156 \\
\hline C -2.885939 & -6.072535 & 1.254491 & C 5.870010 & 0.834607 & -2.475731 & 5.556468 & -0.101898 & -2.946762 \\
\hline C -3.517289 & -4.848302 & 1.290392 & C 5.870174 & -0.833057 & 2.475581 & 5.556404 & 0.103416 & 2.9465250 \\
\hline C - 2.818981 & -3.654127 & 0.987857 & H - 0.418632 & 4.236320 & 2.052863 & 6.102760 & -1.552144 & 3.270795 \\
\hline C $\quad 2.730768$ & -3.633750 & -0.863109 & H $\quad 0.806163$ & 5.937599 & 3.3407150 & 6.800386 & -0.637434 & 1.927993 \\
\hline
\end{tabular}

\section{QH-E}

\begin{tabular}{|c|c|c|c|c|c|c|c|c|}
\hline 0.678462 & 0.173385 & -0.084534 & C -4.791960 & 3.556060 & -0.635040 & H -1.780465 & -7.722457 & -0.356977 \\
\hline C -0.678457 & -0.173386 & 0.084521 & C -4.586100 & 4.490568 & -1.631802 & H -3.413010 & -5.865764 & -0.328601 \\
\hline C -1.626129 & 0.891949 & 0.311665 & C -3.297639 & 4.665123 & -2.171851 & 5.793202 & -3.381425 & 0.249406 \\
\hline C - 1.285561 & 2.191823 & -0.131829 & C -2.229286 & 3.944572 & -1.670570 & 5.422289 & -5.066817 & 2.018088 \\
\hline C $\quad 0.109895$ & 2.589221 & -0.031082 & C 3.885213 & -1.712620 & -0.801916 & 3.140714 & -5.361557 & 2.990732 \\
\hline 1.074900 & 1.556625 & 0.124410 & C -2.817484 & -3.233177 & -0.525344 & 1.240381 & -4.084302 & 2.093630 \\
\hline 1.626133 & -0.891948 & -0.311685 & C -3.885212 & 1.712621 & 0.801885 & H - 1.426428 & 4.895734 & 0.391436 \\
\hline 1.285568 & -2.191823 & 0.131813 & C 2.817479 & 3.233175 & 0.525387 & H - 0.662877 & 7.186618 & 0.504591 \\
\hline C - -0.109888 & -2.589222 & 0.031073 & C $\quad 2.905271$ & -0.738070 & -0.968203 & $\mathrm{H} \quad 1.780457$ & 7.722455 & 0.357021 \\
\hline C - -1.074895 & -1.556626 & -0.124414 & C $\quad 3.311341$ & 0.282055 & -1.897304 & H 3.413005 & 5.865764 & 0.328679 \\
\hline 2.394721 & -3.011951 & 0.617719 & C 4.555032 & 0.090933 & -2.418714 & H -5.793191 & 3.381439 & -0.249439 \\
\hline C -0.526952 & -3.989979 & -0.141745 & 5.300954 & -1.365951 & -1.7684480 & H - 5.422260 & 5.066847 & -2.018101 \\
\hline C $\quad 0.526954$ & 3.989977 & 0.141746 & $\mathrm{~S} \quad 4.454311$ & 3.4296180 & 1.1271660 & H -3.140676 & 5.361597 & -2.990720 \\
\hline C - -2.394709 & 3.011959 & -0.617732 & C 4.538967 & 1.729043 & 1.563003 & H - 1.240352 & 4.084327 & -2.093618 \\
\hline C $\quad 0.367584$ & -5.080645 & -0.306561 & C 3.392402 & 1.077373 & 1.230001 & H $\quad 2.681531$ & 1.116371 & -2.181772 \\
\hline C - -0.066541 & -6.390328 & -0.385135 & C 2.400246 & 1.907455 & 0.591701 & H 3.246066 & 0.032923 & 1.467429 \\
\hline C - 1.438757 & -6.691575 & -0.325316 & C -2.905272 & 0.738070 & 0.968174 & H - 2.681540 & -1.116379 & 2.181733 \\
\hline C - -2.347257 & -5.656171 & -0.290300 & C -3.311348 & -0.282059 & 1.897268 & H - 3.246074 & -0.032938 & -1.467426 \\
\hline C - 1.918516 & -4.307007 & -0.259051 & C -4.555040 & -0.090937 & 2.418674 & H $\quad 4.653625$ & 1.781868 & -3.716024 \\
\hline 3.721098 & -2.778681 & 0.137110 & S -5.300957 & 1.365951 & 1.768411 & H 5.507126 & 0.359599 & -4.341304 \\
\hline 4.791975 & -3.556045 & 0.635016 & S - 4.454324 & -3.429626 & -1.127102 & 6.222672 & 1.317889 & -3.038863 \\
\hline 4.586125 & -4.490544 & 1.631790 & C -4.538981 & -1.729055 & -1.562962 & 6.645179 & 1.250329 & 1.577288 \\
\hline 3.297669 & -4.665093 & 2.171852 & C -3.392410 & -1.077384 & -1.229982 & 5.993088 & 1.683050 & 3.163129 \\
\hline C 2.229310 & -3.944551 & 1.670571 & C -2.400248 & -1.907460 & -0.591682 & 5.607590 & 0.107329 & 2.443654 \\
\hline C -0.367588 & 5.080641 & 0.306538 & C $\quad 5.275745$ & 0.927693 & -3.431723 & H -5.993088 & -1.683052 & -3.163100 \\
\hline 0.066533 & 6.390324 & 0.385127 & C $\quad 5.761804$ & 1.167829 & 2.222577 & H -6.645201 & -1.250380 & -1.577254 \\
\hline 1.438751 & 6.691572 & 0.325347 & C -5.761822 & -1.167848 & -2.222533 & H -5.607625 & -0.107341 & -2.443584 \\
\hline 2.347253 & 5.656170 & 0.290351 & C -5.275759 & -0.927700 & 3.431676 & H -4.653645 & -1.781881 & 3.715971 \\
\hline 1.918515 & 4.307006 & 0.259085 & H $\quad 1.426421$ & -4.895740 & -0.391491 & H -5.507137 & -0.359611 & 4.341261 \\
\hline C -3.721089 & 2.778689 & -0.137133 & $\begin{array}{ll}\text { H } & 0.662865\end{array}$ & -7.186624 & -0.504620 & H - 6.222688 & -1.317888 & 3.038812 \\
\hline
\end{tabular}

\section{$\mathbf{T S}_{\mathrm{A}-\mathrm{C}}$}

\begin{tabular}{|c|c|c|c|c|c|c|c|c|c|}
\hline C -0.710929 & 0.287436 & -0.326098 & $\mathrm{C}$ & 4.815330 & 4.781936 & -1.2946640 & H -6.072859 & -2.910567 & -1.257817 \\
\hline 0.611081 & -0.155759 & -0.241059 & $\mathrm{C}$ & 3.560205 & 5.174851 & -1.7802990 & Н -5.976988 & -5.125866 & -0.168105 \\
\hline
\end{tabular}




\begin{tabular}{|c|c|c|c|c|c|c|c|c|}
\hline 1.656957 & 0.800462 & -0.5482020 & C -4.056402 & -1.097543 & -1.384017 & H -3.862054 & -5.847571 & 0.956564 \\
\hline 1.377254 & 2.200159 & -0.6910320 & 2.115292 & -3.359327 & 1.135196 & Н - 1.864552 & -4.462314 & 0.829996 \\
\hline 0.036317 & 2.666196 & -0.2520520 & 4.062269 & 1.183179 & -0.7692960 & H -0.346280 & 7.335682 & -0.922438 \\
\hline C -0.910080 & 1.665238 & 0.078653 & C -1.913425 & 3.124635 & 1.771305 & H - 1.375832 & 7.677038 & 1.341961 \\
\hline C -1.773571 & -0.641066 & -0.606585 & C -3.025768 & -0.185490 & -1.176702 & H - 2.127953 & 5.676006 & 2.592731 \\
\hline C -1.555901 & -2.034039 & -0.453336 & C -3.357389 & 1.116334 & -1.698518 & H 5.953989 & 3.104321 & -0.6013530 \\
\hline C -0.205275 & -2.494603 & -0.193097 & C -4.590170 & 1.188860 & -2.272139 & 5.667247 & 5.454442 & -1.3399120 \\
\hline C $\quad 0.797079$ & -1.546353 & 0.127333 & S -5.419704 & -0.359453 & -2.196834 & 3.436367 & 6.136279 & -2.2708650 \\
\hline C - -2.742777 & -2.897985 & -0.382077 & S - -2.954375 & 3.117802 & 3.177135 & H - 2.688707 & 1.966631 & -1.669271 \\
\hline C $\quad 0.238150$ & -3.889926 & -0.319376 & C -3.374397 & 1.434265 & 2.902848 & H -2.867630 & -0.088912 & 1.500089 \\
\hline C -0.396935 & 4.052157 & 0.087956 & C -2.744139 & 0.941400 & 1.801694 & 2.745735 & -1.827796 & -1.468945 \\
\hline C $\quad 2.529829$ & 3.062079 & -1.0351190 & C -1.897868 & 1.896189 & 1.124938 & 2.695528 & -0.124530 & 1.871871 \\
\hline C -0.365188 & -4.794275 & -1.227484 & 2.991058 & 0.306006 & -0.8464270 & H -4.566661 & 3.234115 & -2.877818 \\
\hline C $\quad 0.097859$ & -6.088726 & -1.379789 & 3.418663 & -1.008828 & -1.256201 & H -5.472291 & 2.180328 & -3.979130 \\
\hline 1.193577 & -6.544909 & -0.624503 & 4.765774 & -1.139987 & -1.398169 & H -6.176199 & 2.648669 & -2.426351 \\
\hline 1.844107 & -5.673504 & 0.227004 & 5.591158 & 0.3747270 & 1.0603110 & H -5.291903 & 1.189838 & 3.869725 \\
\hline 1.408942 & -4.335922 & 0.367502 & 3.380015 & -3.6625260 & 2.304935 & H -4.437121 & -0.316920 & 3.498438 \\
\hline C - 3.996184 & -2.437130 & -0.895302 & 3.583114 & -1.937984 & 2.596106 & H -3.914660 & 0.691066 & 4.859783 \\
\hline C -5.137352 & -3.271613 & -0.838755 & 2.735236 & -1.205488 & 1.822985 & 4.544074 & -0.354166 & 3.653117 \\
\hline C -5.088730 & -4.502121 & -0.217150 & C 1.887393 & -1.997307 & 0.969464 & 4.365016 & -1.836108 & 4.609609 \\
\hline C -3.893946 & -4.915290 & 0.399627 & C -5.238790 & 2.371871 & -2.924442 & 5.599958 & -1.742438 & 3.347334 \\
\hline C - -2.758234 & -4.131609 & 0.319256 & C -4.303071 & 0.715914 & 3.834045 & 4.848385 & -3.175469 & -2.029759 \\
\hline C -0.529639 & 6.490204 & -0.265166 & 4.575626 & -1.446869 & 3.605781 & 6.178295 & -2.184482 & -2.660016 \\
\hline C -1.138285 & 6.680175 & 0.981640 & 5.539733 & -2.363000 & -1.786141 & H 6.187435 & -2.708783 & -0.970840 \\
\hline C - 1.529994 & 5.563691 & 1.692235 & H - 1.198920 & -4.456695 & -1.831030 & C 2.470729 & 4.327763 & -1.6617580 \\
\hline C -1.245485 & 4.259735 & 1.227388 & H -0.382123 & -6.749240 & -2.096502 & H $\quad 1.565180$ & 4.618624 & -2.1530280 \\
\hline 3.868732 & 2.594742 & -0.8040880 & 1.548783 & -7.565601 & -0.736349 & C -0.189634 & 5.215012 & -0.690681 \\
\hline C 4.967196 & 3.482541 & -0.8554180 & 2.727105 & -5.999123 & 0.770959 & H $\quad 0.138473$ & 5.116466 & -1.7083030 \\
\hline
\end{tabular}

\section{TS $_{\text {A-D }}$}

$\begin{array}{lccccccc}\text { C }-0.784714 & -0.143248 & 0.418340 & \text { C } & 4.699272 & -3.546009 & -0.575962 \\ \text { C } & 0.613625 & 0.124158 & 0.3944930 & \text { C } & 4.642270 & -4.157500 & -1.813272 \\ \text { C } & 1.511606 & -1.005275 & 0.516031 & \text { C } & 3.464227 & -4.070124 & -2.577906 \\ \text { C } & 1.177089 & -2.154908 & -0.221658 & \text { C } & 2.342027 & -3.447230 & -2.061593 \\ \text { C }-0.239797 & -2.478242 & -0.323097 & \text { C } & -3.904391 & 1.927989 & -0.374739 \\ \text { C }-1.170175 & -1.562034 & 0.267146 & \text { C } & 2.963242 & 3.081468 & -0.1774940 \\ \text { C }-1.684738 & 0.993952 & 0.203205 & \text { C } & 3.652376 & -2.020486 & 1.106530 \\ \text { C }-1.133588 & 2.163840 & -0.369826 & \text { C }-2.997708 & -3.120249 & -0.241901 \\ \text { C } & 0.238282 & 2.496441 & -0.1032140 & \text { C }-3.123936 & 1.148987 & 0.474310 \\ \text { C } & 1.146545 & 1.437718 & 0.1161320 & \text { C }-5.101460 & 1.375615 & 1.752469 \\ \text { C }-1.933296 & 2.904887 & -1.358584 & \text { S }-5.520031 & 2.176318 & 0.2517500 \\ \text { C } & 0.683417 & 3.882268 & 0.0268620 & \text { S }-4.592490 & -3.623830 & 0.290611 \\ \text { C }-0.741542 & -3.761209 & -0.850241 & \text { C }-4.481187 & -2.601111 & 1.711182 \\ \text { C } & 2.337374 & -2.875264 & -0.766057 & \text { C }-2.485799 & -2.102684 & 0.558899 \\ \text { C }-0.219009 & 4.945529 & 0.277918 & \text { C } & 2.708387 & -1.017156 & 1.311197 \\ \text { C } & 0.228221 & 6.235394 & 0.4983910 & \text { C } & 3.050918 & -0.162314 & 2.414759 \\ \text { C } & 1.605667 & 6.521362 & 0.4864540 & \text { C } & 4.209704 & -0.511276 & 3.039725 \\ \text { C } & 2.514545 & 5.501832 & 0.2783820 & \text { S } & 4.949701 & -1.916062 & 2.2732490 \\ \text { C } & 2.077216 & 4.176816 & 0.0595660 & \text { S } & 4.640751 & 3.2504430 & -0.651587\end{array}$

$\begin{array}{lcc}\text { H -5.157057 } & 3.099167 & -2.532329 \\ \text { H -4.049024 } & 4.503422 & -4.247465 \\ \text { H - } 1.576981 & 4.837757 & -4.157455 \\ \text { H - } 0.252844 & 3.820424 & -2.368649 \\ \text { H } 1.086528 & -4.919568 & -0.816596 \\ \text { H } 0.212006 & -6.859419 & -1.969101 \\ \text { H -2.185473 } & -6.931096 & -2.697023 \\ \text { H -3.710056 } & -5.127193 & -1.944871 \\ \text { H } 5.627553 & -3.544565 & -0.010419 \\ \text { H } 5.516725 & -4.664738 & -2.211367 \\ \text { H } 3.434872 & -4.482105 & -3.582722 \\ \text { H } 1.444594 & -3.380199 & -2.665553 \\ \text { H } 2.435924 & 0.669692 & 2.7386900 \\ \text { H } 3.481542 & -0.162931 & -0.867864 \\ \text { H -5.600957 } & 0.852891 & 3.762152 \\ \text { H -6.454245 } & 2.289589 & 3.172678 \\ \text { H -6.952449 } & 0.682501 & 2.632509 \\ \text { H -5.814016 } & -3.609807 & 3.084623 \\ \text { H -5.257944 } & -2.011090 & 3.610139\end{array}$




\begin{tabular}{|c|c|c|c|c|c|c|c|c|}
\hline C -3.337748 & 2.687934 & -1.448991 & C $\quad 4.767769$ & 1.528402 & -0.9550200 & H -6.494170 & -2.151634 & 2.351286 \\
\hline C -4.085620 & 3.277179 & -2.486262 & 3.598855 & 0.890893 & -0.6685650 & H $\quad 6.329544$ & 1.377404 & -2.4438550 \\
\hline C -3.465813 & 4.059082 & -3.445678 & C $\quad 2.547767$ & 1.750349 & -0.1797800 & 6.871561 & 1.062233 & -0.7906580 \\
\hline C -2.076016 & 4.253052 & -3.389672 & C -6.080543 & 1.299178 & 2.885039 & 5.896316 & -0.145393 & -1.642481 \\
\hline C -1.327497 & 3.684971 & -2.371475 & C -5.569792 & -2.597630 & 2.740151 & 4.249224 & 0.980353 & 4.5639210 \\
\hline C $\quad 0.054963$ & -4.900520 & -1.128105 & 6.034291 & 0.929013 & -1.4871170 & 4.921732 & -0.576211 & 5.080522 \\
\hline C -0.447139 & -6.018602 & -1.771736 & C 4.846650 & 0.123223 & 4.2386030 & H 5.859526 & 0.482925 & 4.0196410 \\
\hline C -1.797358 & -6.072536 & -2.156052 & H -1.281722 & 4.740050 & 0.320512 & C -3.320608 & -1.887539 & 1.705617 \\
\hline C -2.641188 & -5.054391 & -1.761462 & H - 0.489776 & 7.026013 & 0.697694 & H - 3.004313 & -1.308099 & 2.557622 \\
\hline C -2.147532 & -3.941261 & -1.044220 & 1.955028 & 7.535187 & 0.6617120 & C -3.820679 & 0.910377 & 1.712104 \\
\hline C $\quad 3.581347$ & -2.853343 & -0.054311 & H $\quad 3.581231$ & 5.709859 & 0.2981520 & H - 3.342457 & 0.516074 & 2.595123 \\
\hline
\end{tabular}

\section{$\mathrm{TS}_{\mathrm{B}-\mathrm{C}}$}

\begin{tabular}{|c|c|c|c|c|c|c|c|c|c|c|c|}
\hline $\mathrm{C}$ & 0.141679 & -0.783898 & -0.193543 & $\mathrm{C}$ & -6.367612 & 1.700636 & -1.979772 & $\mathrm{H}$ & 5.225928 & -3.012201 & -3.208383 \\
\hline C & -0.130430 & 0.584047 & -0.262625 & $\mathrm{C}$ & -6.114668 & 0.332694 & -2.149714 & $\mathrm{H}$ & 6.704916 & -1.147495 & -3.850757 \\
\hline $\mathrm{C}$ & -1.438802 & 0.977402 & -0.714033 & $\mathrm{C}$ & 2.944204 & -2.809835 & -1.723106 & $\mathrm{H}$ & 6.165348 & 1.176800 & -3.107026 \\
\hline $\mathrm{C}$ & -2.524239 & 0.037365 & -0.796351 & $\mathrm{C}$ & 1.744421 & 3.297983 & 1.627379 & $\mathrm{H}$ & 4.252124 & 1.587444 & -1.632525 \\
\hline $\mathrm{C}$ & -2.299055 & -1.284513 & -0.155322 & $\mathrm{C}$ & -2.881278 & 2.839540 & -1.374298 & $\mathrm{H}$ & -6.166122 & -3.993127 & -0.544597 \\
\hline $\mathrm{C}$ & -0.972917 & -1.581062 & 0.253893 & $\mathrm{C}$ & -1.789723 & -2.937451 & 2.117827 & $\mathrm{H}$ & -6.037929 & -4.688773 & 1.864973 \\
\hline C & 1.474083 & -1.231932 & -0.554333 & $\mathrm{C}$ & 1.726231 & -2.555765 & -1.084241 & $\mathrm{H}$ & -3.952120 & -4.193081 & 3.107102 \\
\hline C & 2.492662 & -0.255700 & -0.640734 & $\mathrm{C}$ & 0.874894 & -3.719001 & -1.098242 & $\mathrm{H}$ & -5.444652 & 3.597661 & -1.612221 \\
\hline C & 2.279494 & 0.976885 & 0.120691 & $\mathrm{C}$ & 1.433568 & -4.815035 & -1.682044 & $\mathrm{H}$ & -7.350218 & 2.117638 & -2.181258 \\
\hline C & 0.950892 & 1.431578 & 0.247677 & S & 3.047915 & -4.469123 & -2.278991 & $\mathrm{H}$ & -6.884662 & -0.324856 & -2.543649 \\
\hline $\mathrm{C}$ & 3.638875 & -0.483304 & -1.496682 & $\mathrm{~S}$ & -1.283519 & -3.682271 & 3.618414 & $\mathrm{H}$ & -0.120706 & -3.749100 & -0.679657 \\
\hline $\mathrm{C}$ & 3.354714 & 1.640131 & 0.847228 & $\mathrm{C}$ & 0.401286 & -3.308631 & 3.280671 & $\mathrm{H}$ & 1.496666 & -2.321850 & 1.730260 \\
\hline $\mathrm{C}$ & -3.304404 & -2.289243 & 0.302196 & $\mathrm{C}$ & 0.533357 & -2.654374 & 2.093832 & $\mathrm{H}$ & 0.464485 & 3.095853 & -1.572846 \\
\hline $\mathrm{C}$ & -3.803016 & 0.572433 & -1.315131 & $\mathrm{C}$ & -0.713669 & -2.431349 & 1.404688 & $\mathrm{H}$ & -1.544596 & 2.641363 & 1.315168 \\
\hline $\mathrm{C}$ & 4.650896 & 1.078411 & 1.003204 & $\mathrm{C}$ & -1.603924 & 2.331860 & -1.205795 & $\mathrm{H}$ & -0.172435 & -6.194079 & -1.416961 \\
\hline $\mathrm{C}$ & 5.656823 & 1.738285 & 1.679392 & $\mathrm{C}$ & -0.597882 & 3.298238 & -1.570193 & $\mathrm{H}$ & 0.772919 & -6.481864 & -2.889075 \\
\hline $\mathrm{C}$ & 5.419839 & 3.003704 & 2.251948 & $\mathrm{C}$ & -1.097177 & 4.508629 & -1.941234 & $\mathrm{H}$ & 1.429312 & -6.944500 & -1.314201 \\
\hline C & 4.155176 & 3.546623 & 2.191343 & S & -2.854499 & 4.516990 & -1.883068 & $\mathrm{H}$ & 1.511961 & -4.769018 & 4.424071 \\
\hline C & 3.098967 & 2.868371 & 1.533420 & S & 1.185456 & 4.630049 & 2.623635 & $\mathrm{H}$ & 2.451279 & -3.373176 & 3.867743 \\
\hline $\mathrm{C}$ & 3.896996 & -1.791422 & -2.012268 & $\mathrm{C}$ & -0.491684 & 4.169876 & 2.370585 & $\mathrm{H}$ & 1.330100 & -3.205712 & 5.229623 \\
\hline $\mathrm{C}$ & 5.027941 & -2.009863 & -2.838079 & $\mathrm{C}$ & -0.588472 & 3.080885 & 1.561537 & $\mathrm{H}$ & -2.564168 & 4.493462 & 2.759147 \\
\hline $\mathrm{C}$ & 5.849096 & -0.966181 & -3.206419 & $\mathrm{C}$ & 0.677700 & 2.575218 & 1.091303 & $\mathrm{H}$ & -1.509285 & 4.960245 & 4.106440 \\
\hline $\mathrm{C}$ & 5.551522 & 0.343780 & -2.775944 & $\mathrm{C}$ & 0.838232 & -6.181614 & -1.835984 & $\mathrm{H}$ & -1.618698 & 5.988309 & 2.672214 \\
\hline $\mathrm{C}$ & 4.476172 & 0.574400 & -1.944111 & $\mathrm{C}$ & 1.476398 & -3.685751 & 4.254117 & $\mathrm{H}$ & 0.731064 & 5.515778 & -2.380507 \\
\hline $\mathrm{C}$ & -5.358938 & -3.639906 & 0.091090 & $\mathrm{C}$ & -1.600855 & 4.945728 & 3.013358 & $\mathrm{H}$ & -0.637731 & 6.074722 & -3.360256 \\
\hline $\mathrm{C}$ & -5.268716 & -4.061757 & 1.423285 & $\mathrm{C}$ & -0.341358 & 5.732329 & -2.361094 & $\mathrm{H}$ & -0.501895 & 6.566997 & -1.667338 \\
\hline $\mathrm{C}$ & -4.119716 & -3.757550 & 2.125548 & $\mathrm{H}$ & 4.857232 & 0.099739 & 0.591500 & $\mathrm{C}$ & -4.870999 & -0.191160 & -1.840152 \\
\hline $\mathrm{C}$ & -3.101805 & -2.958453 & 1.558099 & $\mathrm{H}$ & 6.633282 & 1.271663 & 1.775869 & $\mathrm{H}$ & -4.700180 & -1.216082 & -2.086992 \\
\hline $\mathrm{C}$ & -4.025072 & 1.992483 & -1.381621 & $\mathrm{H}$ & 6.217792 & 3.531023 & 2.767382 & $\mathrm{C}$ & -4.395257 & -2.796974 & -0.442260 \\
\hline $\mathrm{C}$ & -5.311322 & 2.519286 & -1.638866 & $\mathrm{H}$ & 3.947805 & 4.495013 & 2.679984 & $\mathrm{H}$ & -4.431221 & -2.631584 & -1.503804 \\
\hline \multicolumn{12}{|c|}{$\mathbf{T S}_{\mathbf{B}-\mathrm{D}}$} \\
\hline & 657507 & 0.615373 & -0.295943 & $\mathrm{C}$ & 5.449686 & 1.198543 & -2.332358 & & 6.652997 & -1.399282 & -1.678773 \\
\hline
\end{tabular}

\section{TS $_{\text {B-D }}$}

C -0.657507

C 0.318915

0.615373

C 1.620440

$\begin{array}{ll}-0.433402 & -0.378451\end{array}$

C 2.182880

$\begin{array}{ll}-0.064117 & -0.893687\end{array}$

$1.121557 \quad-0.417901$

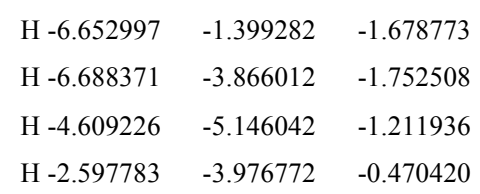




\begin{tabular}{|c|c|c|c|c|c|c|c|c|}
\hline C $\quad 1.322384$ & 1.899388 & 0.453163 & C -4.464454 & 0.136990 & -1.051644 & 3.601359 & 1.383602 & 1.852943 \\
\hline C -0.061336 & 1.914698 & 0.112412 & 0.786737 & -3.641347 & 1.609219 & 4.166429 & 2.183275 & 4.107194 \\
\hline C - -2.058013 & 0.108093 & -0.405705 & 3.562620 & -0.429335 & -2.320776 & 2.638343 & 3.746073 & 5.308301 \\
\hline C -2.267469 & -1.272082 & -0.113539 & C -0.230922 & 3.856101 & 1.639039 & 0.542131 & 4.495658 & 4.224494 \\
\hline C -1.243802 & -2.021071 & 0.582424 & C -3.245270 & 0.813841 & -0.875607 & 5.987134 & 0.572472 & -3.039974 \\
\hline C $\quad 0.089243$ & -1.703643 & 0.265317 & C -4.655426 & 2.557751 & -1.640266 & 6.956624 & 2.719499 & -2.310129 \\
\hline C -3.456517 & -1.987505 & -0.557780 & S -5.749484 & 1.195599 & -1.591401 & 5.674368 & 4.192052 & -0.750669 \\
\hline C -1.561659 & -3.003741 & 1.602907 & S -0.883540 & 5.476350 & 1.802086 & 3.500169 & 3.480922 & 0.112207 \\
\hline C 1.758131 & 2.504608 & 1.701620 & C -1.525535 & 5.422399 & 0.178033 & 0.903487 & -2.510000 & -2.354198 \\
\hline C $\quad 3.476521$ & 1.571656 & -0.906086 & C -0.628135 & 3.243798 & 0.449903 & 3.113025 & -1.675923 & 0.181771 \\
\hline C - -2.835166 & -3.083049 & 2.226860 & C 2.305048 & -0.844178 & -1.883829 & H -4.304350 & 4.629840 & -2.020633 \\
\hline C - 3.107266 & -4.026698 & 3.195769 & 1.859212 & -2.038078 & -2.550097 & H -5.535140 & 3.914483 & -3.076428 \\
\hline C - -2.113485 & -4.943831 & 3.593464 & C $\quad 2.749287$ & -2.526260 & -3.457039 & H -5.924034 & 4.289496 & -1.393459 \\
\hline C -0.848265 & -4.856410 & 3.052608 & 4.193561 & -1.521334 & -3.540680 & H - 1.590952 & 7.523700 & -0.335073 \\
\hline C -0.539209 & -3.873145 & 2.080963 & 2.182329 & -4.546091 & 2.160428 & H - 2.372404 & 6.439385 & -1.498430 \\
\hline C -4.606170 & -1.272100 & -0.978323 & C 3.270555 & -3.434414 & 1.357963 & H -3.169196 & 6.818654 & 0.036119 \\
\hline C - 5.775521 & -1.965836 & -1.379187 & C 2.585106 & -2.452089 & 0.710203 & 5.121899 & -3.613651 & 2.459959 \\
\hline C -5.791897 & -3.342159 & -1.432380 & C 1.147872 & -2.554407 & 0.804345 & 5.062940 & -4.582345 & 0.982501 \\
\hline C -4.623253 & -4.064163 & -1.113438 & C -5.132832 & 3.916138 & -2.055630 & 5.258165 & -2.825186 & 0.877124 \\
\hline C -3.489070 & -3.402947 & -0.689883 & C -2.197432 & 6.615312 & -0.431923 & 1.654145 & -4.223764 & -4.143317 \\
\hline C 2.944606 & 2.087612 & 2.351480 & C 4.755217 & -3.627143 & 1.426057 & 2.675049 & -3.487474 & -5.391337 \\
\hline C 3.256162 & 2.530325 & 3.626251 & C 2.618806 & -3.740429 & -4.325439 & H 3.407929 & -4.474482 & -4.120727 \\
\hline C $\quad 2.392367$ & 3.406051 & 4.306210 & H -3.605744 & -2.376257 & 1.945412 & C -3.405302 & 2.183983 & -1.256561 \\
\hline C $\quad 1.218538$ & 3.824884 & 3.700688 & H -4.089430 & -4.054547 & 3.659259 & H - 2.607791 & 2.887449 & -1.229842 \\
\hline C $\quad 0.884963$ & 3.385349 & 2.406865 & H - 2.333473 & -5.695541 & 4.346516 & C -1.286166 & 4.207921 & -0.397780 \\
\hline C 4.189445 & 0.764873 & -1.852928 & H - 0.065388 & -5.527601 & 3.395280 & H - 1.445619 & 4.065933 & -1.458502 \\
\hline
\end{tabular}

\section{TS $_{\text {C-E }}$}

\begin{tabular}{|c|c|c|c|c|c|}
\hline 0.723518 & 0.109117 & -0.449952 & C -3.003171 & -5.253383 & -0.220670 \\
\hline C -0.687808 & -0.004616 & -0.267081 & C - -2.329300 & -6.323601 & 0.325174 \\
\hline C - 1.260984 & -1.343915 & -0.335041 & C - 1.018905 & -6.137842 & 0.803075 \\
\hline C -0.551809 & -2.390009 & 0.279563 & C -0.433792 & -4.886967 & 0.778875 \\
\hline $\begin{array}{ll}\text { C } & 0.838727\end{array}$ & -2.102566 & 0.610969 & C $\quad 3.243721$ & 2.966638 & 0.002494 \\
\hline 1.479253 & -1.136856 & -0.204810 & C -3.676166 & 2.240467 & 0.425607 \\
\hline 1.321392 & 1.448510 & -0.373329 & C -3.030286 & -2.893969 & -0.985265 \\
\hline C $\quad 0.494019$ & 2.516220 & 0.072043 & C 3.584543 & -2.322447 & 0.289788 \\
\hline C -0.933978 & 2.420323 & -0.065837 & C $\quad 2.711187$ & 1.839084 & -0.625309 \\
\hline C -1.516596 & 1.136772 & -0.011735 & C 4.825942 & 1.998124 & -1.672703 \\
\hline C $\quad 1.081241$ & 3.614159 & 0.855087 & S 4.878137 & 3.308893 & -0.514954 \\
\hline C - 1.786254 & 3.584527 & -0.293466 & S 5.050351 & -2.911989 & -0.466353 \\
\hline C $\quad 1.636971$ & -2.725209 & 1.656593 & C 4.566607 & -2.250029 & -2.018683 \\
\hline C -1.119099 & -3.739722 & 0.297868 & C $\quad 2.823925$ & -1.552729 & -0.588183 \\
\hline C - 1.283992 & 4.785477 & -0.851707 & C - -2.464999 & -1.623797 & -1.066342 \\
\hline C -2.117567 & 5.850561 & -1.139692 & C -3.194290 & -0.766564 & -1.965815 \\
\hline C -3.498328 & 5.760018 & -0.884077 & C -4.288140 & -1.351365 & -2.523734 \\
\hline C -4.026026 & 4.589696 & -0.372396 & S - 4.477230 & -3.012532 & -1.971229 \\
\hline C -3.193093 & 3.485504 & -0.086151 & S -5.256681 & 1.988075 & 1.134150 \\
\hline 2.490027 & 3.804819 & 0.874644 & C -4.852374 & 0.330377 & 1.545655 \\
\hline 3.070502 & 4.792220 & 1.700716 & C -3.593682 & 0.013903 & 1.132589 \\
\hline
\end{tabular}

$\begin{array}{cccc}\text { H } & 4.149753 & 4.920727 & 1.692062 \\ \text { H } & 2.742157 & 6.318479 & 3.170469 \\ \text { H } & 0.276300 & 5.918465 & 3.256382 \\ \text { H }-0.750893 & 4.223827 & 1.840089 \\ \text { H }-0.026818 & -3.096003 & 2.983092 \\ \text { H } & 1.355638 & -3.937644 & 4.840867 \\ \text { H } & 3.835192 & -4.065998 & 4.603277 \\ \text { H } & 4.916156 & -3.287767 & 2.517188 \\ \text { H }-3.998014 & -5.392703 & -0.635862 \\ \text { H }-2.794423 & -7.304897 & 0.359747 \\ \text { H }-0.454688 & -6.982741 & 1.188205 \\ \text { H } & 0.580618 & -4.793282 & 1.134865 \\ \text { H }-2.902726 & 0.250984 & -2.193772 \\ \text { H }-3.159194 & -0.954420 & 1.336022 \\ \text { H } & 5.732606 & 0.904308 & -3.264434 \\ \text { H } & 6.236147 & 2.603332 & -3.199379 \\ \text { H } & 6.882672 & 1.443470 & -2.032070 \\ \text { H } & 4.883938 & -2.076728 & -4.123773 \\ \text { H } & 6.373203 & -1.962144 & -3.172009 \\ \text { H } & 5.603318 & -3.533792 & -3.418345 \\ \text { H }-6.101449 & -0.117603 & 3.253808\end{array}$




$\begin{array}{lllllllllll}\mathrm{C} & 2.286114 & 5.567291 & 2.531577 & \mathrm{C}-2.898598 & 1.085566 & 0.463577 & \mathrm{H}-6.758638 & -0.674357 & 1.709730 \\ \mathrm{C} & 0.897624 & 5.349292 & 2.570559 & \mathrm{C}-5.980943 & 1.728386 & -2.588623 & \mathrm{H}-5.392691 & -1.527541 & 2.444241 \\ \mathrm{C} & 0.314290 & 4.394114 & 1.758118 & \mathrm{C} & 5.402756 & -2.469876 & -3.243399 & \mathrm{H}-4.966547 & 0.267268 & -3.736533 \\ \mathrm{C} & 1.050831 & -3.157479 & 2.870022 & \mathrm{C}-5.829959 & -0.538422 & 2.277625 & \mathrm{H}-5.315001 & -1.328900 & -4.426331 \\ \mathrm{C} & 1.829373 & -3.628175 & 3.913415 & \mathrm{C}-5.265122 & -0.756595 & -3.491757 & \mathrm{H}-6.279336 & -0.720432 & -3.075081 \\ \mathrm{C} & 3.228546 & -3.693424 & 3.782615 & \mathrm{H}-0.226056 & 4.861420 & -1.074617 & \mathrm{C} & 3.384044 & -1.579876 & -1.915249 \\ \mathrm{C} & 3.833757 & -3.261554 & 2.614791 & \mathrm{H}-1.703691 & 6.754493 & -1.577749 & \mathrm{H} & 2.864712 & -1.195901 & -2.786314 \\ \mathrm{C} & 3.061618 & -2.758176 & 1.549386 & \mathrm{H}-4.151625 & 6.598388 & -1.109738 & \mathrm{C} & 3.631351 & 1.345811 & -1.611316 \\ \mathrm{C}-2.414632 & -3.966031 & -0.277894 & \mathrm{H}-5.096560 & 4.502167 & -0.205514 & \mathrm{H} & 3.389462 & 0.575693 & -2.317594\end{array}$

\section{$\mathbf{T S}_{\mathbf{D}-\mathbf{E}}$}

\begin{tabular}{|c|c|c|c|c|c|c|c|c|}
\hline 0.654612 & -0.140607 & -0.311762 & C -4.916998 & -4.638529 & -0.578600 & 5.778452 & 3.300785 & 0.695484 \\
\hline C -0.693402 & 0.239233 & -0.200143 & C -4.012428 & -4.658269 & -1.650093 & 5.190729 & 5.155756 & 2.217617 \\
\hline C -1.637581 & -0.817144 & 0.084459 & C 4.008537 & 1.600787 & -0.522255 & 2.793366 & 5.599438 & 2.761781 \\
\hline C -1.410163 & -2.099403 & -0.482598 & C -2.747690 & 3.364232 & -0.811420 & 1.019081 & 4.283443 & 1.689704 \\
\hline C -0.001839 & -2.501738 & -0.630588 & C -3.567141 & -1.700656 & 1.303387 & 0.076922 & -6.668996 & -2.804333 \\
\hline C $\quad 0.959160$ & -1.551775 & -0.179409 & C $\quad 2.368209$ & -3.377934 & 0.646973 & 1.935943 & -7.517145 & -1.355442 \\
\hline C 1.664452 & 0.884374 & -0.401566 & C 3.032227 & 0.653125 & -0.818490 & 3.018286 & -5.958633 & 0.231575 \\
\hline C 1.307439 & 2.209157 & -0.058826 & C 3.556891 & -0.425061 & -1.614399 & Н -5.492772 & -3.602242 & 1.205724 \\
\hline C -0.063345 & 2.626449 & -0.299531 & C 4.881964 & -0.305454 & -1.905789 & H -5.778150 & -5.300715 & -0.569355 \\
\hline C -1.065188 & 1.624769 & -0.397613 & S $\quad 5.560958$ & 1.153833 & -1.194417 & H -4.193737 & -5.299112 & -2.508475 \\
\hline C 2.353401 & 3.047243 & 0.524506 & S 3.652153 & -3.735685 & 1.781110 & H $\quad 2.955800$ & -1.248197 & -1.980256 \\
\hline C -0.403217 & 4.018167 & -0.618470 & C 3.791136 & -2.037293 & 2.218285 & H $\quad 2.827880$ & -0.207171 & 1.677285 \\
\hline C $\quad 0.513968$ & -3.863765 & -0.879720 & C $\quad 2.912571$ & -1.273127 & 1.514602 & H -2.251880 & 1.318687 & 2.012748 \\
\hline C -2.608058 & -2.959714 & -0.566253 & C 2.089335 & -2.017590 & 0.592108 & H - 3.484024 & 0.113143 & -1.268827 \\
\hline C $\quad 0.561320$ & 5.015774 & -0.921354 & C - 2.680608 & -0.656168 & 1.067766 & H $\quad 5.135554$ & -2.061856 & -3.089383 \\
\hline C $\quad 0.209542$ & 6.328106 & -1.170026 & C -2.857089 & 0.420835 & 2.007770 & 6.170478 & -0.713439 & -3.593959 \\
\hline C - 1.141846 & 6.719602 & -1.144879 & C -3.840168 & 0.199379 & 2.923019 & 6.567707 & -1.636526 & -2.138825 \\
\hline C -2.115044 & 5.761794 & -0.959639 & S -4.612304 & -1.362154 & 2.664732 & 4.581426 & -2.075064 & 4.230748 \\
\hline C -1.773312 & 4.403263 & -0.749271 & S - 4.433245 & 3.605048 & -1.231816 & 5.803704 & -1.830508 & 2.976508 \\
\hline C 3.729920 & 2.741937 & 0.292141 & C -4.672242 & 1.876833 & -1.429225 & H 4.699903 & -0.512269 & 3.400720 \\
\hline C 4.735360 & 3.535209 & 0.891848 & C -3.530003 & 1.187303 & -1.162246 & Н - 6.345551 & 1.753440 & -2.793392 \\
\hline C 4.406996 & 4.565431 & 1.750942 & C -2.413760 & 2.011718 & -0.767071 & Н -5.941046 & 0.244078 & -1.953360 \\
\hline C 3.054930 & 4.822737 & 2.048591 & C $\quad 5.737250$ & -1.223760 & -2.724848 & H -6.779627 & 1.543955 & -1.092150 \\
\hline C $\quad 2.055930$ & 4.082252 & 1.445443 & C 4.770943 & -1.595426 & 3.262443 & Н -5.330269 & 1.364007 & 3.969643 \\
\hline C $\quad 0.500417$ & -6.038045 & -2.027771 & C -6.005679 & 1.330487 & -1.840008 & H - 4.137082 & 0.599720 & 5.026979 \\
\hline C $\quad 1.555907$ & -6.506764 & -1.233341 & C -4.272634 & 1.084563 & 4.052217 & H -3.680822 & 2.005001 & 4.047637 \\
\hline C $\quad 2.148540$ & -5.640257 & -0.337200 & H 1.605875 & 4.746725 & -0.977851 & C $\quad 0.007087$ & -4.755192 & -1.851694 \\
\hline C 1.680612 & -4.317197 & -0.174886 & H $\quad 0.984138$ & 7.055166 & -1.397369 & Н - 0.737611 & -4.411391 & -2.544547 \\
\hline C -3.643078 & -2.819345 & 0.419412 & H - 1.418372 & 7.756274 & -1.316156 & C -2.897833 & -3.834271 & -1.637287 \\
\hline C -4.744538 & -3.703880 & 0.423896 & H -3.164881 & 6.037064 & -1.014848 & H - 2.293033 & -3.788479 & -2.528598 \\
\hline
\end{tabular}

dithia[6]helicene

\begin{tabular}{|c|c|c|c|c|c|c|c|c|}
\hline 0.001958 & 3.205370 & 0.000140 & C 2.863896 & -0.753809 & -0.018223 & 4.339218 & 1.795147 & 1.632386 \\
\hline 0.001028 & 1.781898 & 0.000332 & C -2.864760 & -0.750511 & 0.018175 & H -4.336893 & 1.800159 & -1.632532 \\
\hline C - 1.280997 & 1.119624 & -0.180489 & C -1.614386 & -0.182302 & 0.335490 & 4.783759 & -0.558967 & 0.984685 \\
\hline C -2.345602 & 1.853633 & -0.794439 & C -1.525194 & -2.176675 & 1.592438 & H -4.784307 & -0.553298 & -0.984801 \\
\hline C -2.206699 & 3.259226 & -1.002881 & S -3.084129 & -2.311469 & 0.786265 & 1.745029 & -3.407592 & -3.356343 \\
\hline C -1.113740 & 3.921577 & -0.528548 & 3.081230 & -2.315017 & -0.786355 & 0.940255 & -4.216943 & -2.006519 \\
\hline
\end{tabular}




$\begin{array}{llllllllllll}\mathrm{C} & 1.118429 & 3.920310 & 0.528464 & \mathrm{C} & 1.522564 & -2.178235 & -1.592475 & \mathrm{H} & 0.074472 & -2.983729 & -2.937354 \\ \mathrm{C} & 2.210773 & 3.256685 & 1.002762 & \mathrm{C} & 1.614079 & -0.184287 & -0.335069 & \mathrm{H}-0.078750 & -2.983976 & 2.938070 \\ \mathrm{C} & 2.347886 & 1.851009 & 0.794489 & \mathrm{C} & 1.049725 & -3.254406 & -2.521682 & \mathrm{H}-1.749939 & -3.407350 & 3.355074 \\ \mathrm{C} & 1.282249 & 1.118110 & 0.181061 & \mathrm{C}-1.053747 & -3.253803 & 2.521227 & \mathrm{H}-0.944214 & -4.215939 & 2.005325 \\ \mathrm{C} & 3.580242 & 1.215133 & 1.113680 & \mathrm{H}-3.028681 & 3.800244 & -1.464938 & \mathrm{C} & 0.886496 & -1.023356 & -1.255637 \\ \mathrm{C}-3.578566 & 1.219336 & -1.113787 & \mathrm{H}-1.054893 & 5.006047 & -0.577695 & \mathrm{H}-0.075806 & -0.761805 & -1.674541 \\ \mathrm{C} & 3.836769 & -0.083836 & 0.746395 & \mathrm{H} & 1.061115 & 5.004876 & 0.577203 & \mathrm{C}-0.887746 & -1.022567 & 1.255754 \\ \mathrm{C}-3.836681 & -0.079426 & -0.746521 & \mathrm{H} & 3.033404 & 3.796923 & 1.464564 & \mathrm{H} & 0.074763 & -0.762203 & 1.674912\end{array}$

\section{TS $_{\text {dithia[6]helicene }}$}

\begin{tabular}{|c|c|c|c|c|c|c|c|c|}
\hline C $\quad 0.000483$ & 3.081682 & -0.922761 & C 2.824831 & -0.550026 & 0.821140 & H 4.130405 & 2.505167 & 1.304066 \\
\hline 0.000255 & 1.697659 & -0.552307 & C -2.825033 & -0.549166 & 0.821076 & H -4.129547 & 2.506461 & 1.304197 \\
\hline C -1.327278 & 1.136520 & -0.196784 & C -1.766182 & -0.239017 & -0.063205 & H $\quad 4.440009$ & 0.160724 & 2.098713 \\
\hline C -2.351940 & 2.080481 & 0.159553 & C -2.126022 & -2.534598 & -0.532849 & H -4.439927 & 0.162088 & 2.098734 \\
\hline C -2.305151 & 3.414186 & -0.336560 & S -3.216718 & -2.258714 & 0.814237 & H $\quad 3.026520$ & -4.234601 & -1.522546 \\
\hline C - 1.196485 & 3.858850 & -0.979795 & S 3.216046 & -2.259693 & 0.814275 & H 1.632137 & -4.638543 & -0.513905 \\
\hline C $\quad 1.197716$ & 3.858431 & -0.979896 & C 2.125256 & -2.535230 & -0.532778 & H 1.390038 & -3.825658 & -2.067504 \\
\hline 2.306266 & 3.413408 & -0.336713 & C $\quad 1.766051$ & -0.239562 & -0.063123 & H -1.391808 & -3.825403 & -2.067843 \\
\hline 2.352602 & 2.079729 & 0.159517 & C 2.043337 & -3.878738 & -1.190807 & H - 1.633022 & -4.637931 & -0.513924 \\
\hline C 1.327590 & 1.136109 & -0.196743 & C -2.044565 & -3.878212 & -1.190719 & H -3.027977 & -4.234068 & -1.521792 \\
\hline C 3.447166 & 1.718112 & 0.995937 & H -3.167110 & 4.058348 & -0.182649 & 1.461757 & -1.394277 & -0.866246 \\
\hline C - 3.446608 & 1.719182 & 0.995975 & H - 1.139687 & 4.873164 & -1.365211 & 0.872747 & -1.339972 & -1.765062 \\
\hline 3.632459 & 0.429777 & 1.424913 & 1.141257 & 4.872738 & -1.365384 & C -1.462260 & -1.393811 & -0.866369 \\
\hline C -3.632324 & 0.430888 & 1.424895 & H 3.168480 & 4.057261 & -0.182959 & H -0.873293 & -1.339520 & -1.765207 \\
\hline
\end{tabular}


Table S5. TD-DFT vertical one-electron excitations (6 states) calculated for optimized structure of QH-A.

\begin{tabular}{|c|c|c|c|c|c|}
\hline excited state & energy & wavelength & oscillator strength $(f)$ & description & \\
\hline \multirow{2}{*}{1} & \multirow{2}{*}{$2.5340 \mathrm{eV}$} & \multirow{2}{*}{$489.29 \mathrm{~nm}$} & \multirow{2}{*}{0.0058} & HOMO-1 -> LUMO & 0.44527 \\
\hline & & & & HOMO -> LUMO+1 & 0.54151 \\
\hline \multirow{2}{*}{2} & \multirow{2}{*}{$2.6066 \mathrm{eV}$} & \multirow{2}{*}{$475.65 \mathrm{~nm}$} & \multirow{2}{*}{0.0877} & HOMO-1 ->LUMO+1 & -0.22863 \\
\hline & & & & HOMO -> LUMO & 0.66579 \\
\hline 3 & $2.9654 \mathrm{eV}$ & $418.11 \mathrm{~nm}$ & 0.0090 & HOMO-2 -> LUMO & 0.69577 \\
\hline \multirow{2}{*}{4} & \multirow{2}{*}{$3.0091 \mathrm{eV}$} & \multirow{2}{*}{$412.02 \mathrm{~nm}$} & \multirow{2}{*}{0.6413} & HOMO-1 -> LUMO & 0.53704 \\
\hline & & & & HOMO -> LUMO+1 & -0.43847 \\
\hline \multirow{3}{*}{5} & \multirow{3}{*}{$3.0382 \mathrm{eV}$} & \multirow{3}{*}{$408.08 \mathrm{~nm}$} & \multirow{3}{*}{0.0000} & HOMO-3 -> LUMO & -0.12231 \\
\hline & & & & HOMO-2 -> LUMO+1 & 0.66331 \\
\hline & & & & HOMO-1 ->LUMO+2 & -0.16013 \\
\hline \multirow{2}{*}{6} & \multirow{2}{*}{$3.0692 \mathrm{eV}$} & \multirow{2}{*}{$403.96 \mathrm{~nm}$} & \multirow{2}{*}{0.4126} & HOMO-1 -> LUMO+1 & 0.65638 \\
\hline & & & & HOMO -> LUMO+1 & 0.22301 \\
\hline
\end{tabular}

Table S6. TD-DFT vertical one-electron excitations (6 states) calculated for optimized structure of QH-B.

\begin{tabular}{|c|c|c|c|c|c|}
\hline excited state & energy & wavelength & oscillator strength $(f)$ & description & \\
\hline \multirow{2}{*}{1} & \multirow{2}{*}{$2.6681 \mathrm{eV}$} & \multirow{2}{*}{$464.69 \mathrm{~nm}$} & \multirow{2}{*}{0.0139} & HOMO-1 -> LUMO & 0.61548 \\
\hline & & & & HOMO -> LUMO+1 & -0.33392 \\
\hline \multirow{2}{*}{2} & \multirow{2}{*}{$2.7294 \mathrm{eV}$} & \multirow{2}{*}{$454.26 \mathrm{~nm}$} & \multirow{2}{*}{0.0851} & HOMO-1 -> LUMO+1 & 0.16329 \\
\hline & & & & HOMO -> LUMO & 0.68437 \\
\hline \multirow{2}{*}{3} & \multirow{2}{*}{$2.9751 \mathrm{eV}$} & \multirow{2}{*}{$416.74 \mathrm{~nm}$} & \multirow{2}{*}{0.0000} & HOMO-2 -> LUMO & 0.68220 \\
\hline & & & & HOMO -> LUMO+2 & 0.15646 \\
\hline \multirow{3}{*}{4} & \multirow{3}{*}{$3.1999 \mathrm{eV}$} & \multirow{3}{*}{$387.46 \mathrm{~nm}$} & \multirow{3}{*}{0.4581} & HOMO-5 -> LUMO & -0.14806 \\
\hline & & & & HOMO-1 -> LUMO & 0.32792 \\
\hline & & & & HOMO -> LUMO+1 & 0.59218 \\
\hline \multirow{3}{*}{5} & \multirow{3}{*}{$3.2164 \mathrm{eV}$} & \multirow{3}{*}{$385.47 \mathrm{~nm}$} & \multirow{3}{*}{0.1935} & HOMO-1 -> LUMO+1 & 0.67076 \\
\hline & & & & HOMO -> LUMO & -0.15814 \\
\hline & & & & HOMO -> LUMO+4 & 0.11857 \\
\hline \multirow{2}{*}{6} & \multirow{2}{*}{3.2763} & \multirow{2}{*}{$378.43 \mathrm{~nm}$} & \multirow{2}{*}{0.0014} & HOMO-3 -> LUMO & 0.67702 \\
\hline & & & & HOMO -> LUMO+3 & -0.15430 \\
\hline
\end{tabular}


(a)

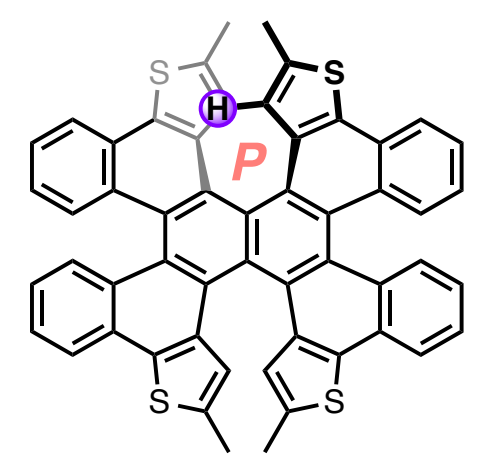

Delete two biaryl units

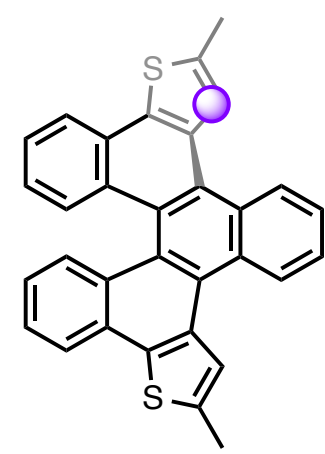

\begin{tabular}{lcccccc}
\hline & QH-A & QH-B & $\Delta\left(\delta \mathrm{H}_{\mathrm{B}}-\delta \mathrm{H}_{\mathrm{A}}\right)$ & $\begin{array}{c}\text { QH-A } \\
\text { (partial) }\end{array}$ & $\begin{array}{c}\text { QH-B } \\
\text { (partial) }\end{array}$ & $\Delta\left(\mathrm{\delta Bq}_{\mathrm{B}}-\mathrm{\delta Bq}_{\mathrm{A}}\right)$ \\
\hline Chemical shifts (ppm) & 6.36 & 7.49 & 1.13 & - & - & - \\
Shielding constants (ppm) & - & - & - & -1.57 & -0.18 & 1.39 \\
\hline
\end{tabular}

(b)
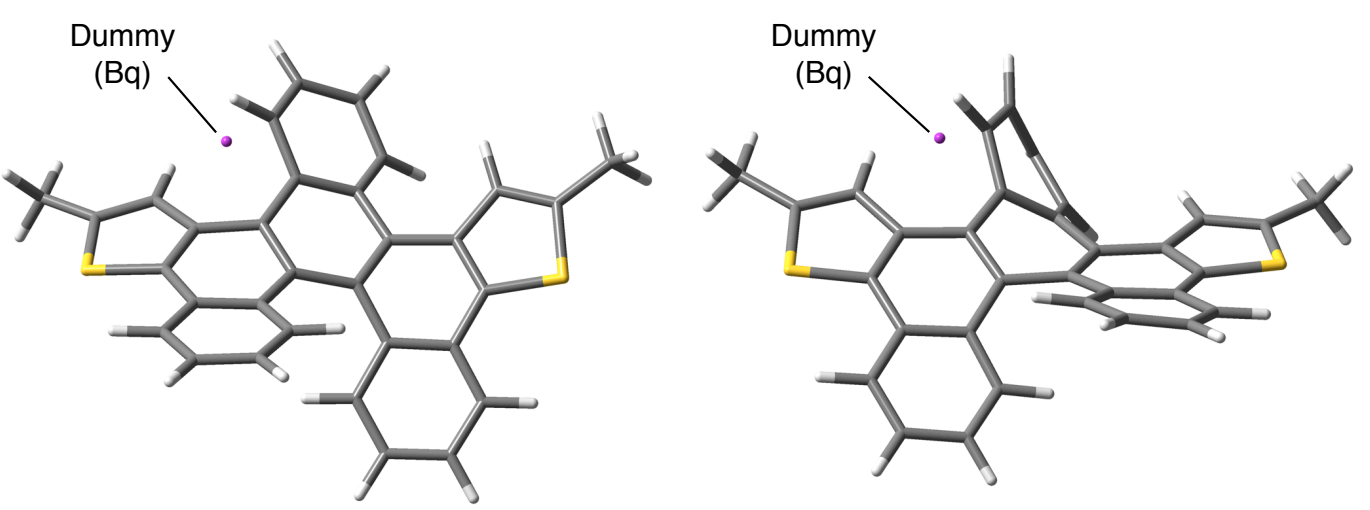

Figure S8. (a) Virtual scheme for the preparation of imperfect structures of QH-A and QH-B removing two biaryl units. Dummy atoms were placed instead of hydrogen atoms at the end of inner helix of dithia[6]helicene substructures. Results of NMR chemical shifts calculations were also summarized. (b) Structures of imperfect form of QH-A and QH-B with dummy atoms (Bq).

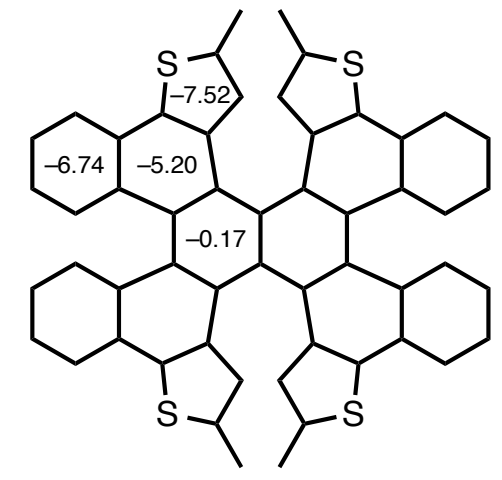

QH-A

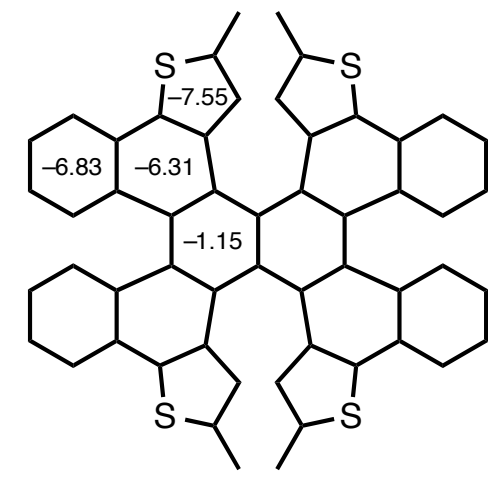

QH-B

Figure S9. NICS(0) of each ring in QH-A and QH-B. 


\section{Kinetic Study}

\section{Thermal conversion from QH-A to QH-B}

Kinetics of the conversion from QH-A to QH-B was studied by monitoring the decreasing integration of QH-A in the ${ }^{1} \mathrm{H}$ NMR spectra in 1,1,2,2-tetrachloroethane- $d_{2}$ at $80.0,84.2$, and $88.4{ }^{\circ} \mathrm{C}$ (Figure S10). All reaction was conducted in NMR tube upon the heating by oil bath under nitrogen. Reactions were stopped at each time for the ${ }^{1} \mathrm{H}$ NMR measurement by cooling the NMR tube in ice bath.

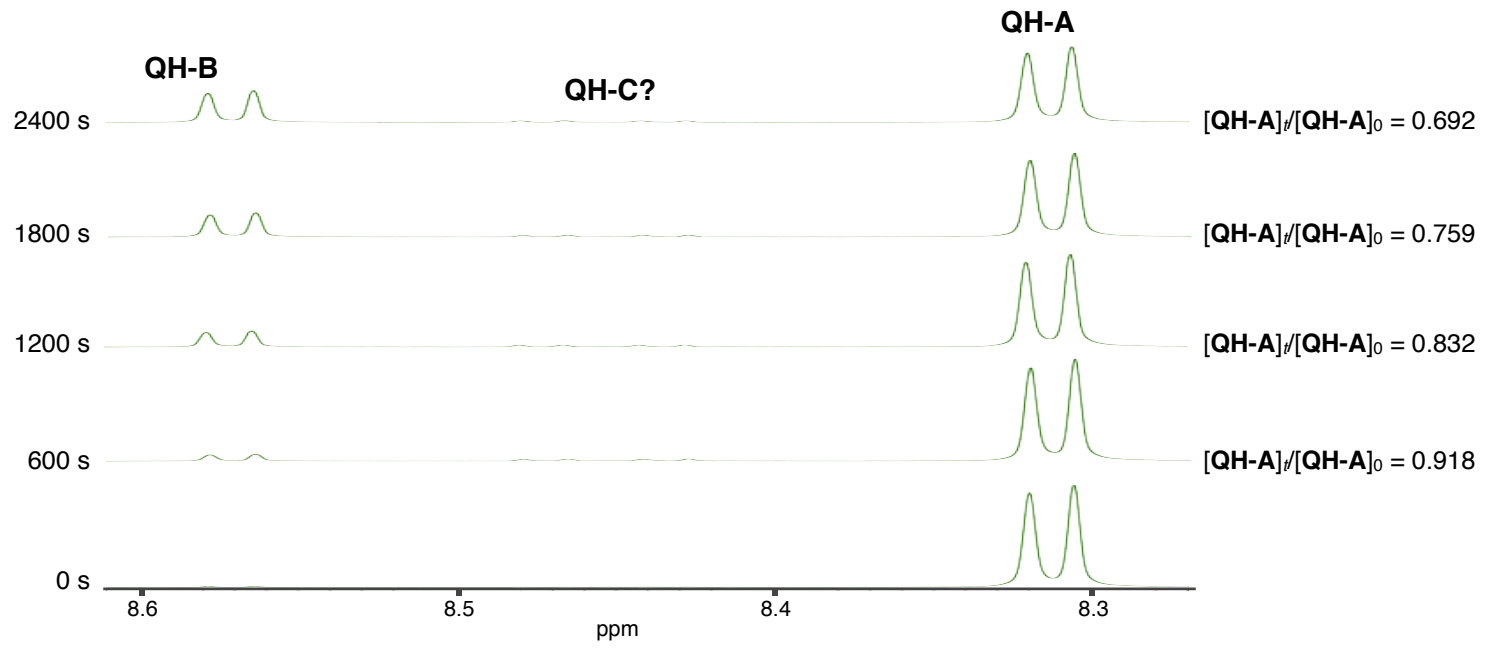

Figure S10. Conversion progress from QH-A to QH-B at $88.4{ }^{\circ} \mathrm{C}$ monitored by ${ }^{1} \mathrm{H}$ NMR.

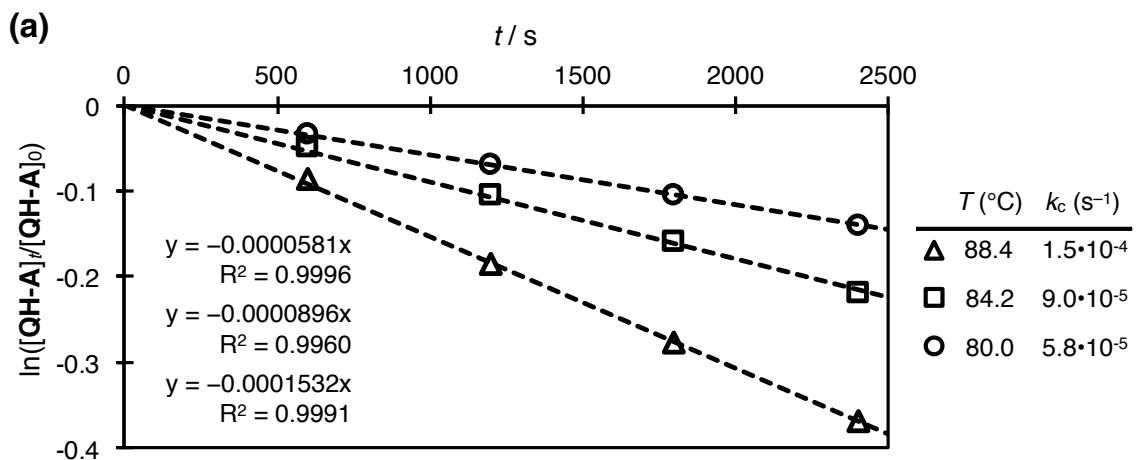

(b)

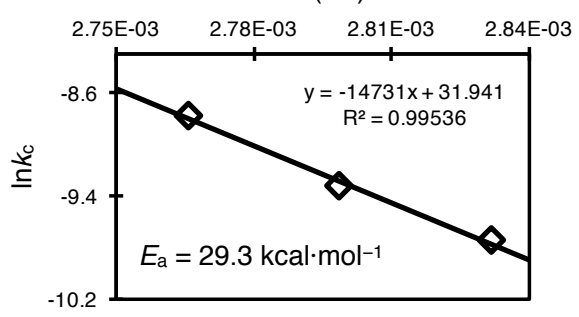

(c) $\quad 1 / T\left(\mathrm{~K}^{-1}\right)$

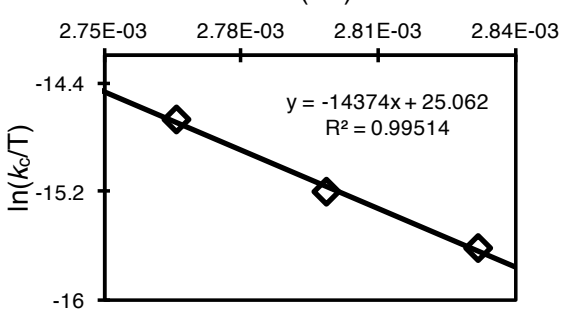

Figure S11. (a) Plots of the decreasing integration of QH-A in the ${ }^{1} \mathrm{H}$ NMR spectra in 1,1,2,2-tetrachloroethane- $d_{2}$ upon heating at $80.0,84.2$, and $88.4^{\circ} \mathrm{C}$. (b) Arrhenius plot and (c) Eyring plot of the thermal conversion from QH-A to QH-B. 


\section{Thermal racemization of QH-B}

Kinetics of the racemization of QH-B was studied by monitoring the decreasing ellipticity ([$[\theta])$ of $(-)$-QH-B $((M, M)-(P, P)-\mathbf{Q H})$ in the $\mathrm{CD}$ spectra in 1,2,4-trichlorobenzene at 164.5, 173.5, and $183.8^{\circ} \mathrm{C}$ (Figure S12). All reactions were conducted in test tube upon the heating by oil bath under nitrogen. Reactions were stopped at each time for the CD measurement by cooling the test tube in ice bath.

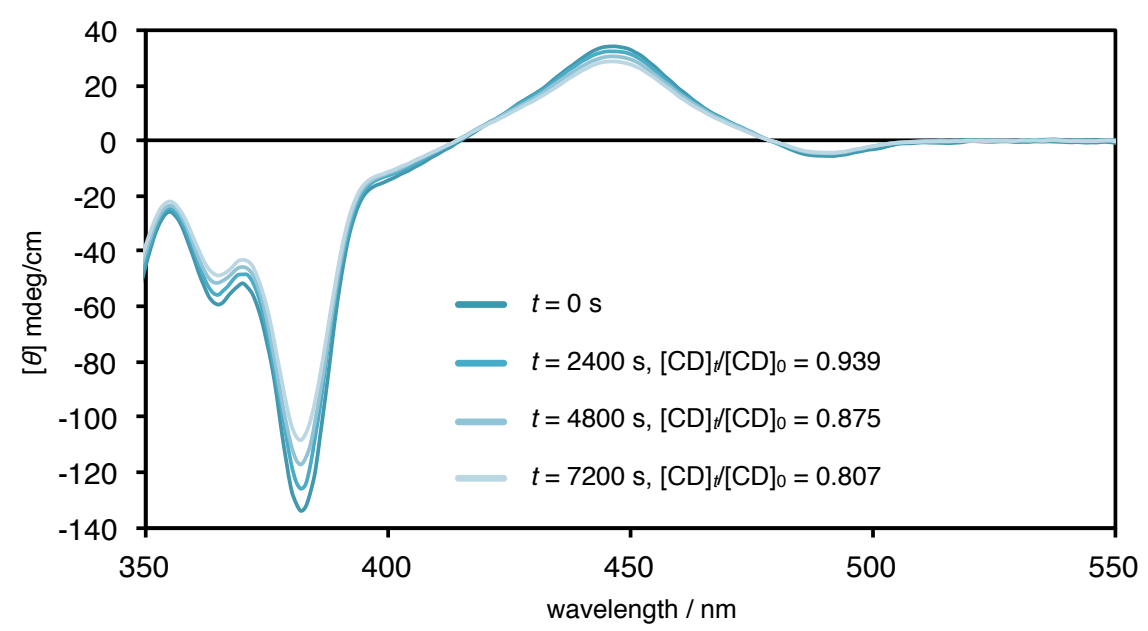

Figure S12. Racemization progress of $(-)-\mathbf{Q H}-\mathbf{B}((M, M)-(P, P)-\mathbf{Q H})$ in 1,2,4-trichlorobenzene at $183.8^{\circ} \mathrm{C}$ monitored by $\mathrm{CD}$ spectra.

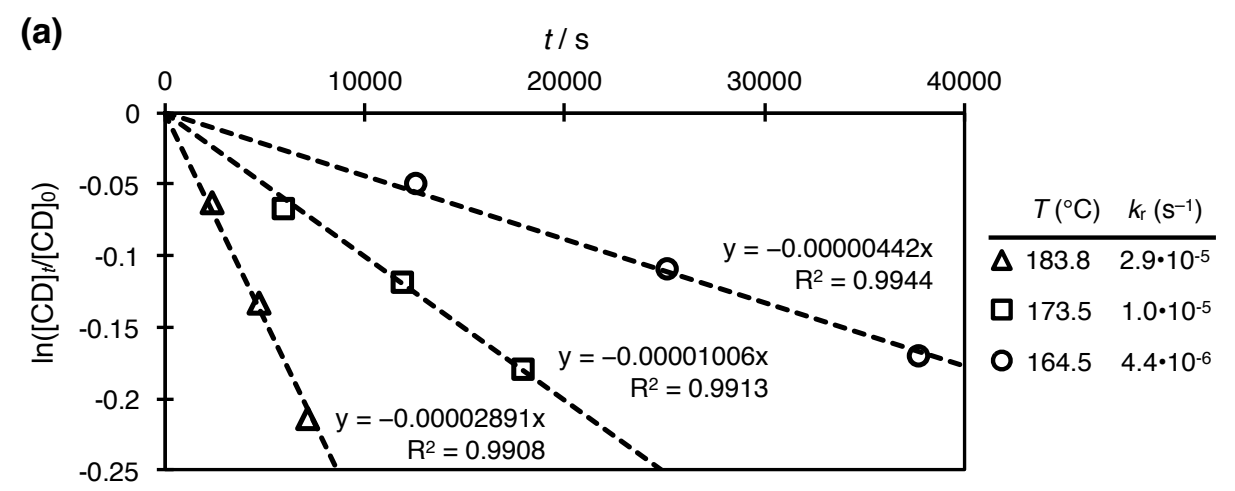

(b)

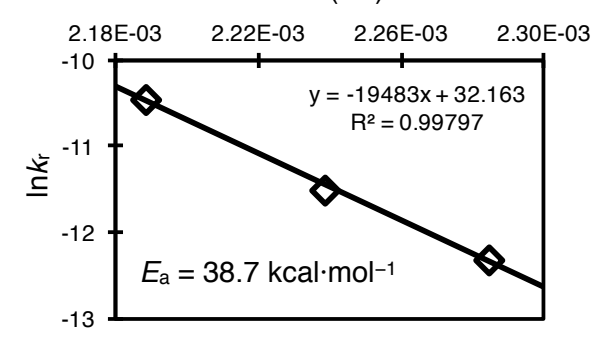

(c)

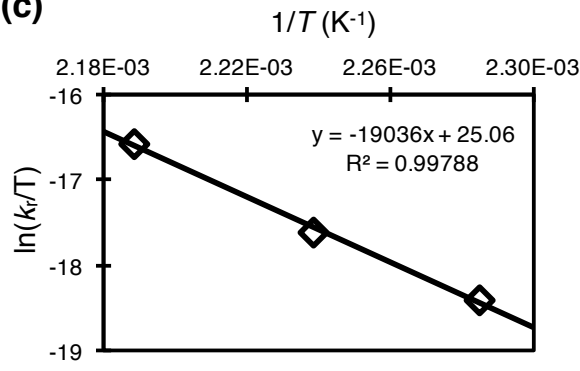

Figure S13. (a) Plots of the decreasing ellipticity at $382 \mathrm{~nm}$ in the CD spectra of enantio-enriched (-)-QH-B in 1,2,4-trichlorobenzene upon heating at $164.5,173.5$, and $183.8^{\circ} \mathrm{C}$. (b) Arrhenius plot and (c) Eyring plot of the thermal racemization of (-)-QH-B. 
Additional theoretical kinetic study

(a)

B3LYP/6-31G(d)

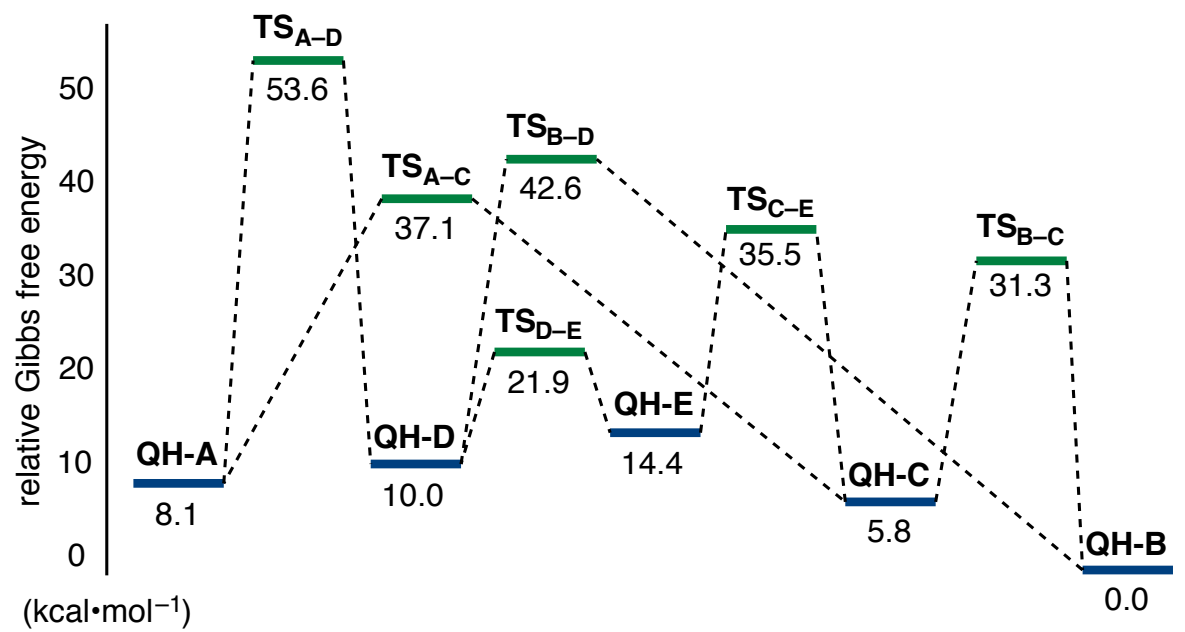

(b)

M06-2X/6-31G(d)

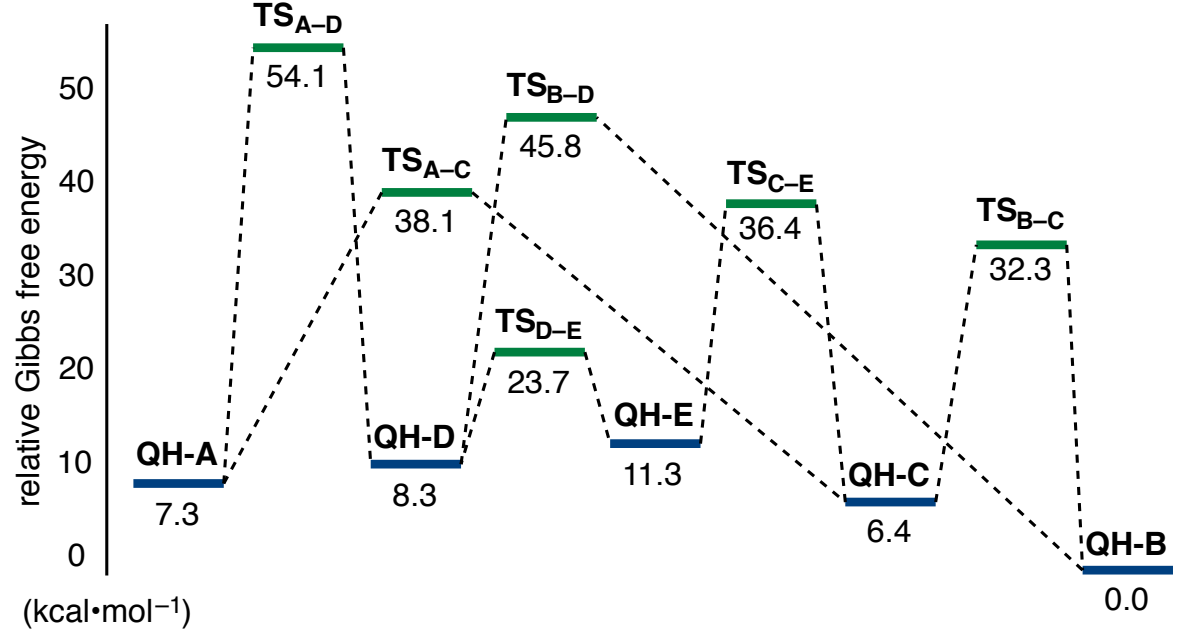

(c)

$\mathrm{BMK} / 6-31 \mathrm{G}(\mathrm{d})$

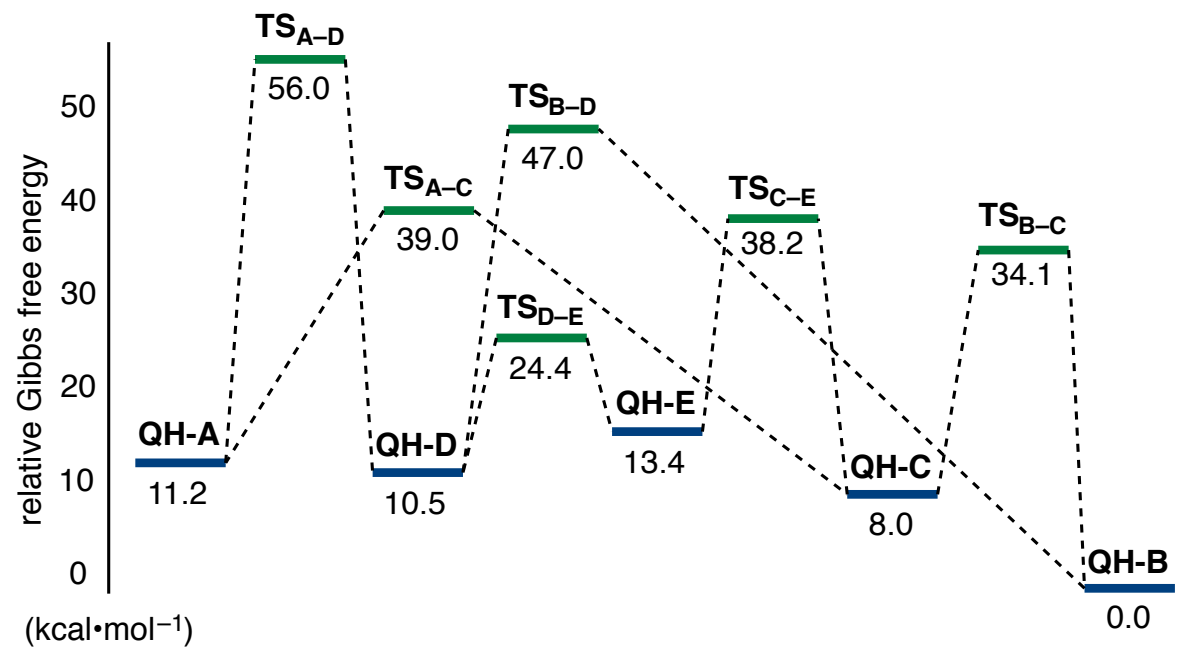

Figure S14. Theoretical study on the interconversion pathway of QHs at the (a) B3LYP/6-31G(d), (b) M06-2X/6-31G(d), and (c) BMK/6-31G(d) levels. 


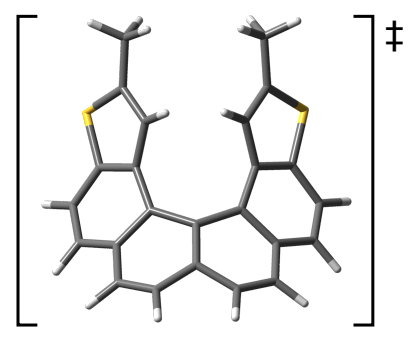

(Gibbs free energy)

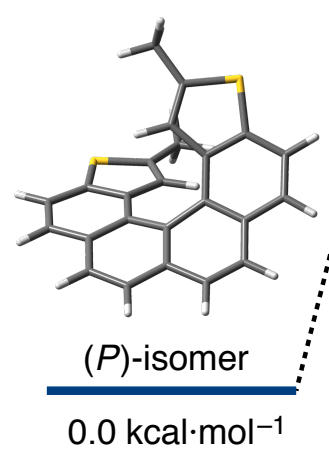

TS ${ }_{\text {dithia[6]helicene }}$

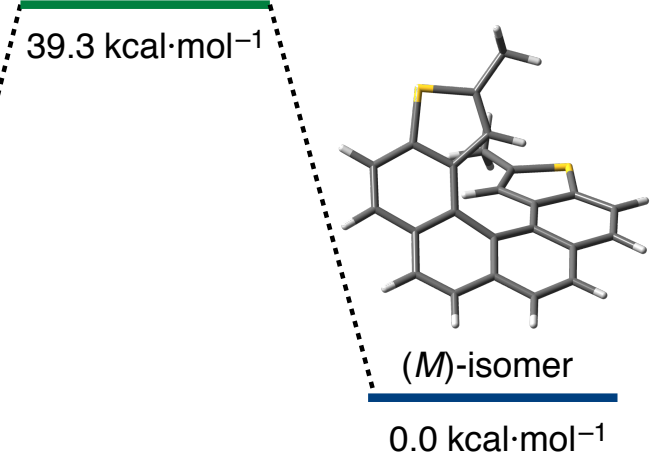

Figure S15. Racemization process of dithia[6]helicene. 


\section{8. ${ }^{1} \mathrm{H}$ and ${ }^{13} \mathrm{C}$ NMR Spectra of Products}

Figure S16. ${ }^{1} \mathrm{H}$ NMR $\left(600 \mathrm{MHz}, \mathrm{CDCl}_{3}\right)$ of 2

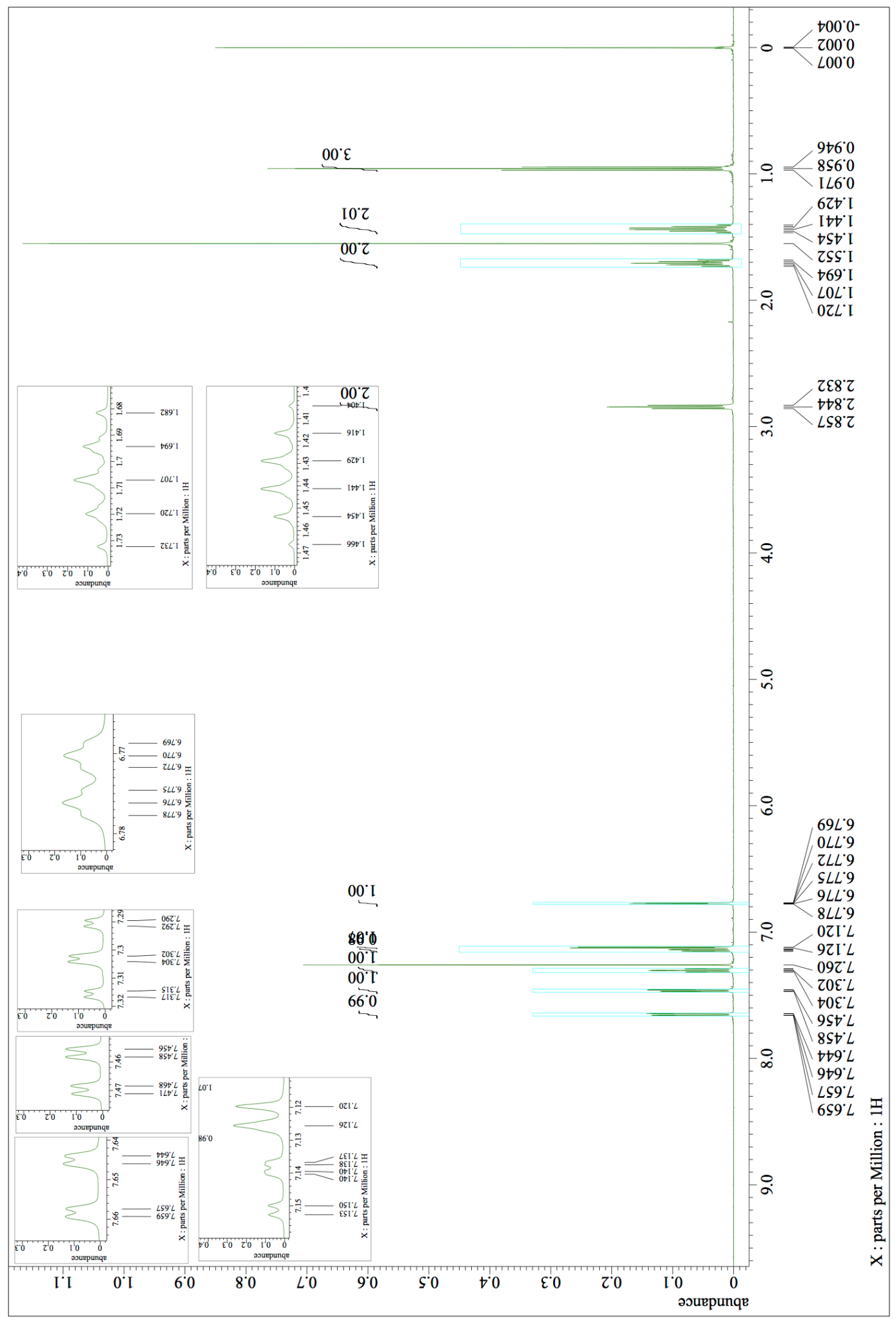


Figure S17. ${ }^{13} \mathrm{C}$ NMR $\left(150 \mathrm{MHz}, \mathrm{CDCl}_{3}\right)$ of 2

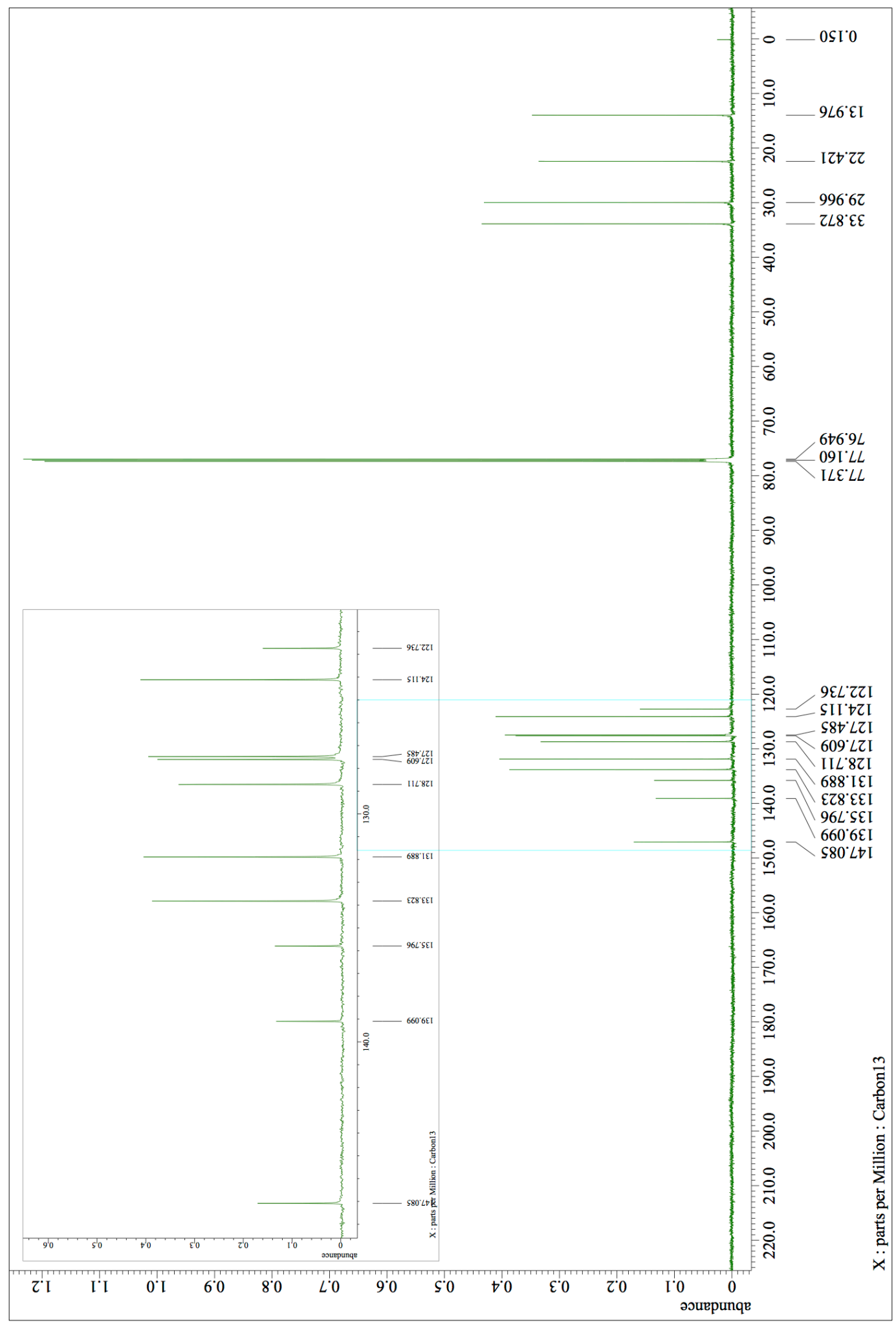


Figure S18. ${ }^{1} \mathrm{H}$ NMR $\left(600 \mathrm{MHz}, \mathrm{C}_{2} \mathrm{D}_{2} \mathrm{Cl}_{4}\right)$ of 5

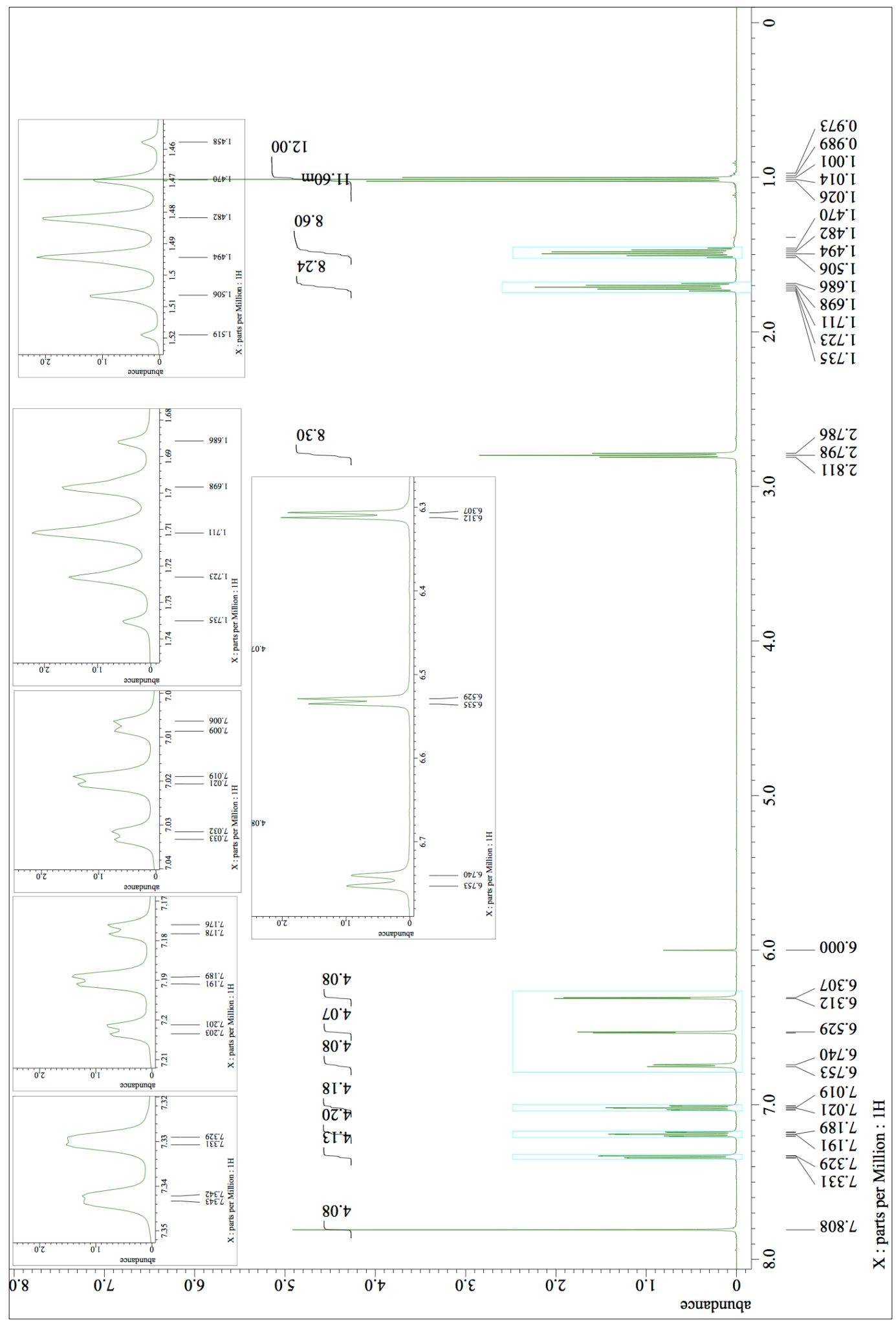


Figure S19. ${ }^{13} \mathrm{C}$ NMR $\left(150 \mathrm{MHz}, \mathrm{C}_{2} \mathrm{D}_{2} \mathrm{Cl}_{4}\right)$ of 5

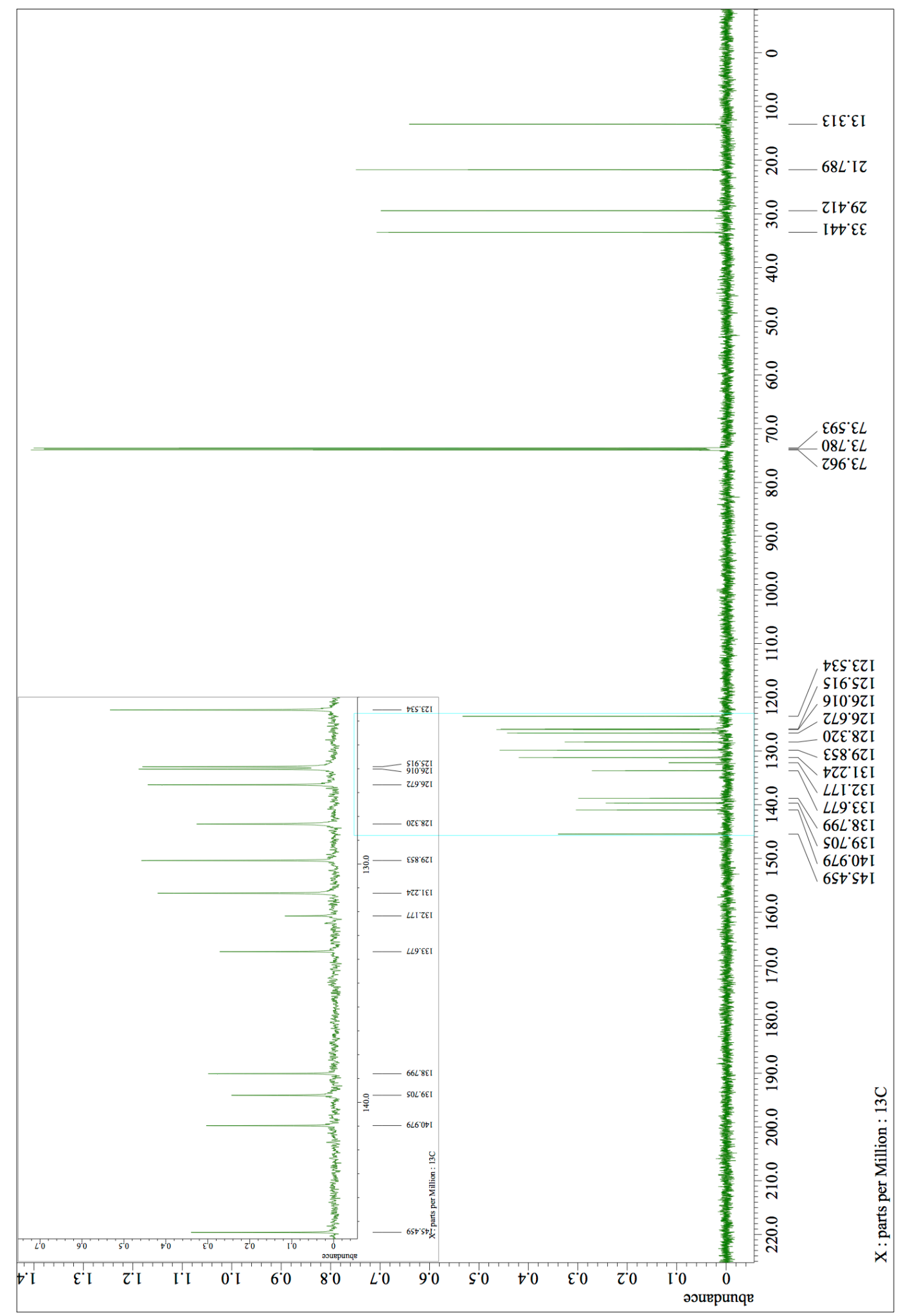


Figure S20. ${ }^{1} \mathrm{H}$ NMR $\left(600 \mathrm{MHz}, \mathrm{CDCl}_{3}\right)$ of $\mathbf{Q H - A}$

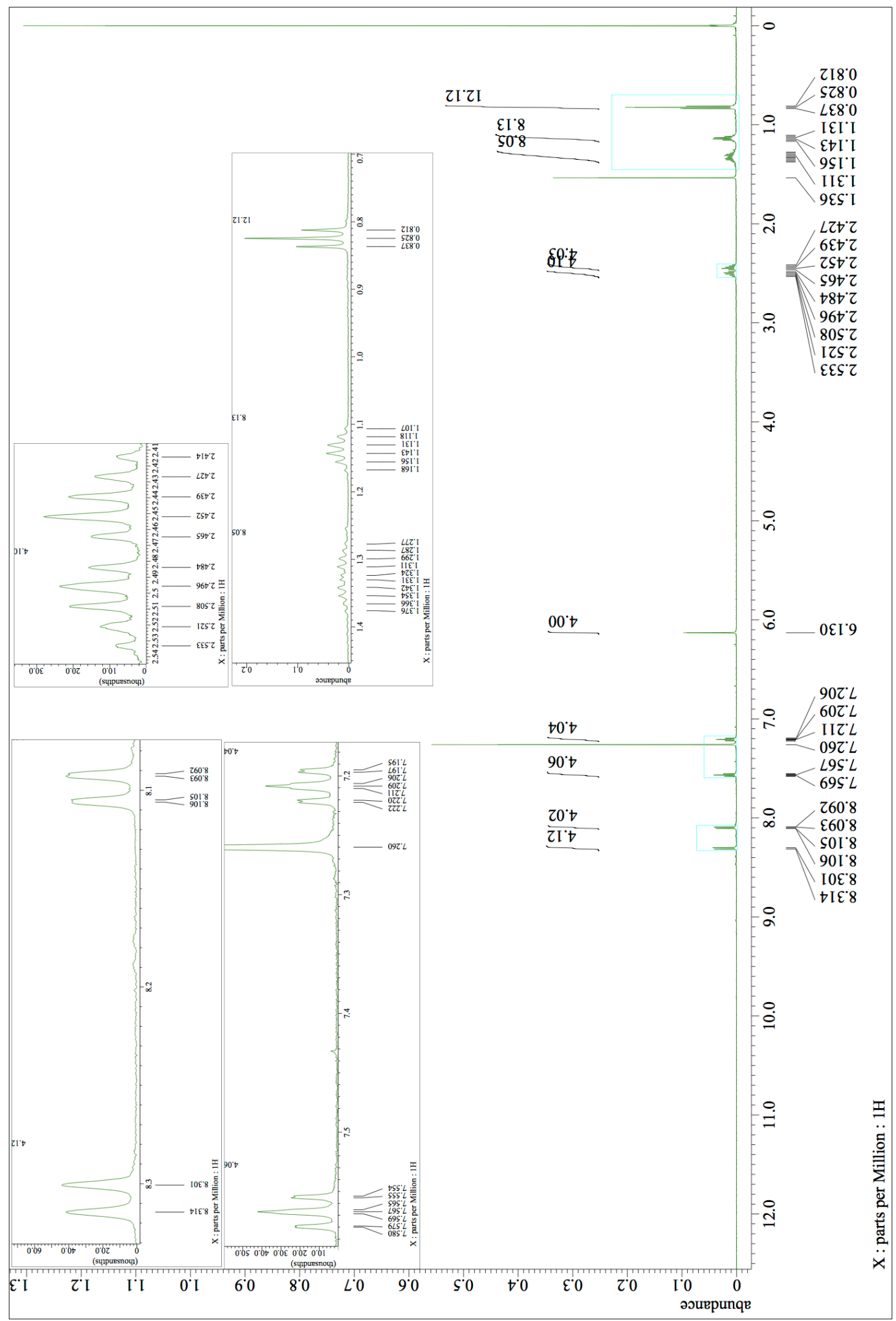


Figure S21. ${ }^{13} \mathrm{C}$ NMR $\left(150 \mathrm{MHz}, \mathrm{CDCl}_{3}\right)$ of QH-A

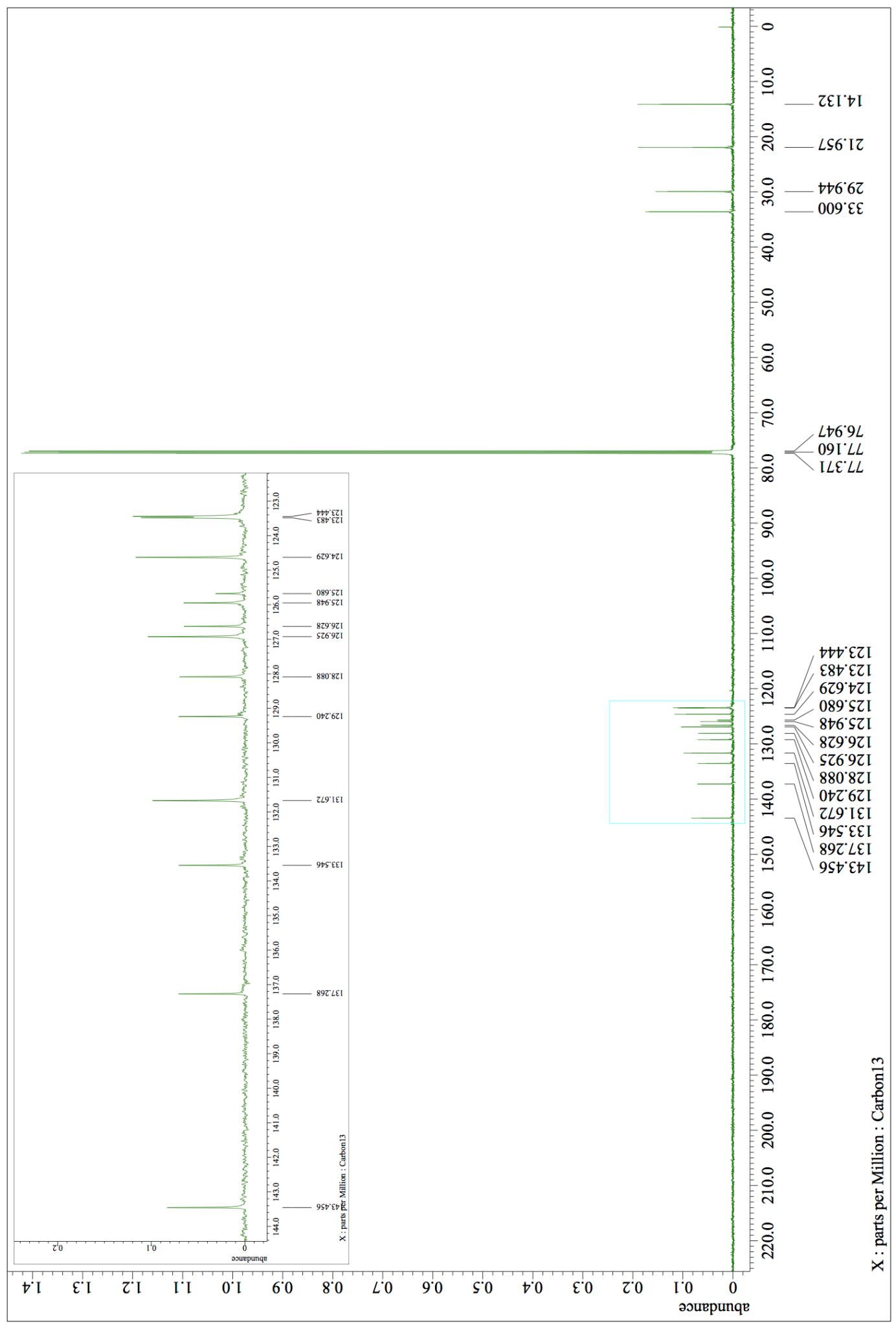


Figure S22. ${ }^{1} \mathrm{H}$ NMR $\left(600 \mathrm{MHz}, \mathrm{CDCl}_{3}\right)$ of $\mathbf{Q H - B}$

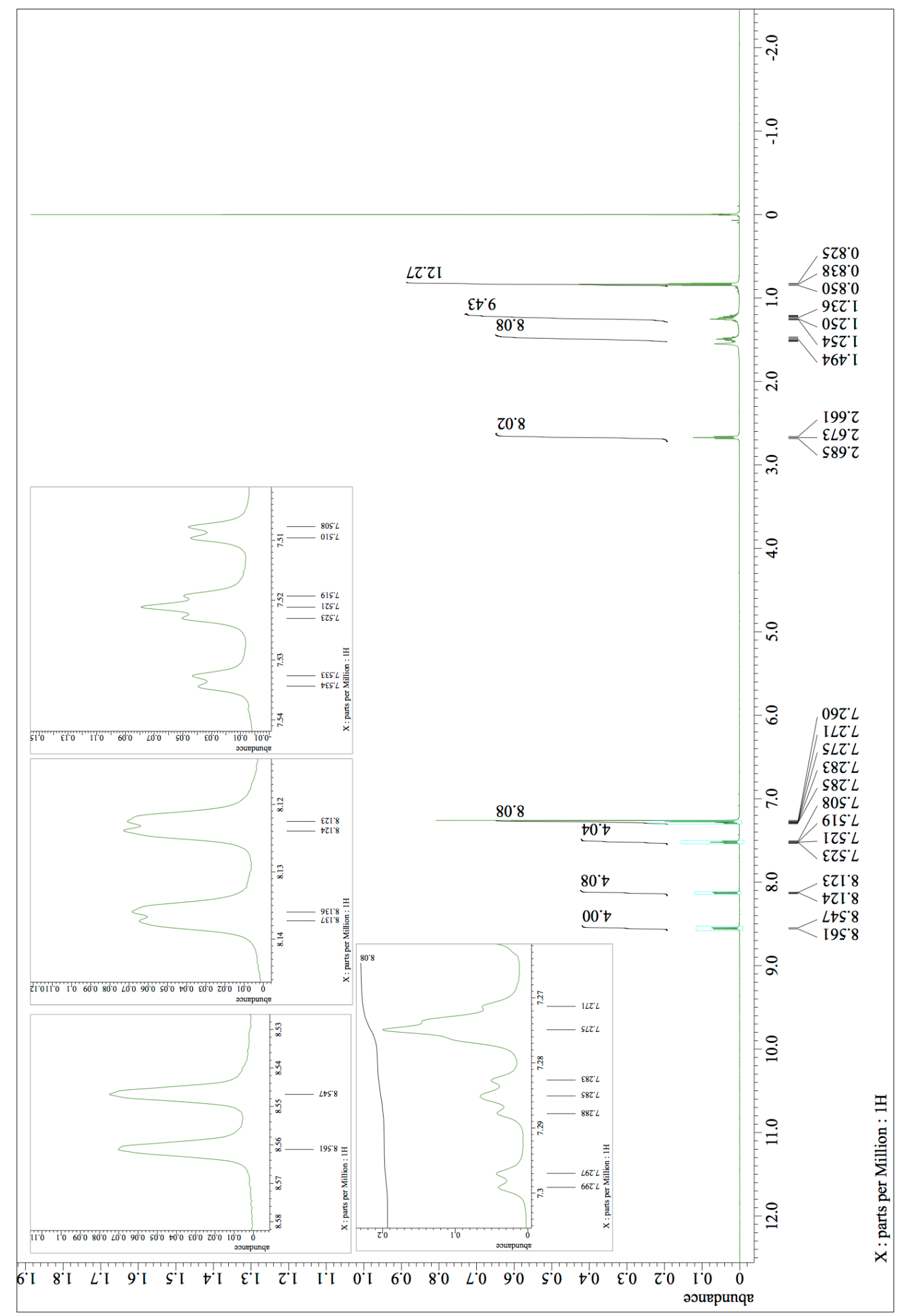


Figure S23. ${ }^{13} \mathrm{C} \mathrm{NMR}\left(150 \mathrm{MHz}, \mathrm{CDCl}_{3}\right)$ of $\mathbf{Q H}-\mathbf{B}$

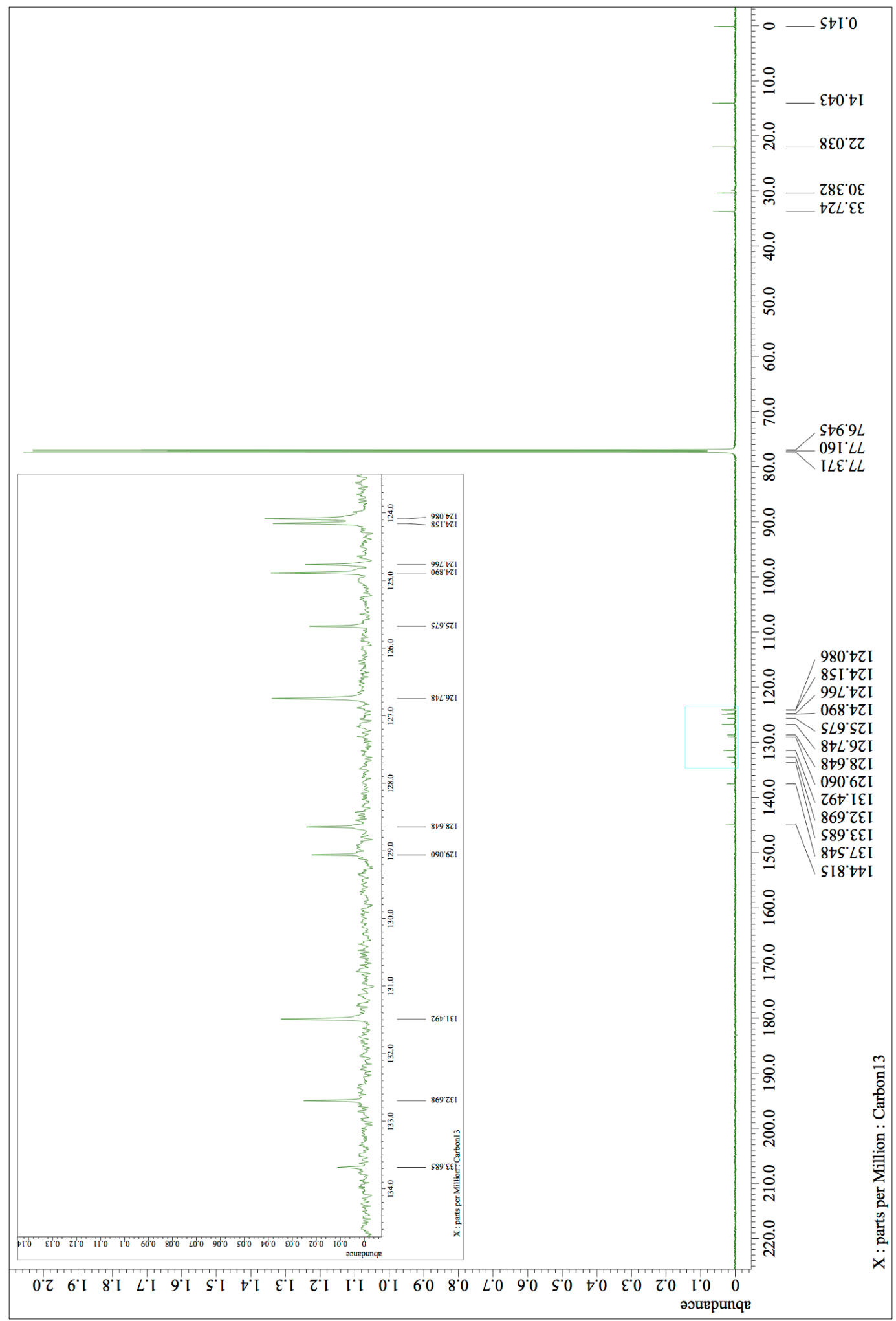




\section{References}

S1) Oyamada, T.; Uchiuzo, H.; Adachi, C.; Takahashi, T.; Akiyama, S. JP Patent 084485, 2007.

S2) Altomare, A.; Burla, M. C.; Camalli, M.; Cascarano, G. L.; Giacovazzo, C.; Guagliardi, A.; Moliterni, A. G. G.; Polidori, G.; Spagna, R. J. Appl. Crystallogr. 1999, 32, 115.

S3) Sheldrick, G. M. University of Göttingen: Göttingen, Germany, 1997.

S4) Frisch, M. J.; Trucks, G. W.; Schlegel, H. B.; Scuseria, G. E.; Robb, M. A.; Cheeseman, J. R.; Scalmani, G.; Barone, V.; Mennucci, B.; Petersson, G. A.; Nakatsuji, H.; Caricato, M.; Li, X.; Hratchian, H. P.; Izmaylov, A. F.; Bloino, J.; Zheng, G.; Sonnenberg, J. L.; Hada, M.; Ehara, M.; Toyota, K.; Fukuda, R.; Hasegawa, J.; Ishida, M.; Nakajima, T.; Honda, Y.; Kitao, O.; Nakai, H.; Vreven, T.; Montgomery, Jr., J. A.; Peralta, J. E.; Ogliaro, F.; Bearpark, M.; Heyd, J. J.; Brothers, E.; Kudin, K. N.; Staroverov, V. N.; Keith, T.; Kobayashi, R.; Normand, J.; Raghavachari, K.; Rendell, A.; Burant, J. C.; Iyengar, S. S.; Tomasi, J.; Cossi, M.; Rega, N.; Millam, J. M.; Klene, M.; Knox, J. E.; Cross, J. B.; Bakken, V.; Adamo, C.; Jaramillo, J.; Gomperts, R.; Stratmann, R. E.; Yazyev, O.; Austin, A. J.; Cammi, R.; Pomelli, C.; Ochterski, J. W.; Martin, R. L.; Morokuma, K.; Zakrzewski, V. G.; Voth, G. A.; Salvador, P.; Dannenberg, J. J.; Dapprich, S.; Daniels, A. D.; Farkas, O.; Foresman, J. B.; Ortiz, J. V.; Cioslowski, J.; Fox, D. J. Gaussian 09, Revision D.01, Gaussian, Inc., Wallingford CT, 2013.

S5) (a) Becke, A. D. J. Chem. Phys. 1993, 98, 5648. (b) Lee, C.; Yang, W.; Parr, R. G. Phys. Rev. B 1988, 37, 785 . 